\title{
SPATIAL AND DYNAMICAL CHAOS GENERATED BY REACTION-DIFFUSION SYSTEMS IN UNBOUNDED DOMAINS
}

\author{
S.V.ZELIK \\ Laboratoire d'Applications des Mathématiques - SP2MI \\ Boulevard Marie et Pierre Curie - Téléport 2 \\ 86962 Chasseneuil Futuroscope Cedex - France
}

\begin{abstract}
We consider in this article a nonlinear reaction-diffusion system with a transport term $\left(L, \nabla_{x}\right) u$, where $L$ is a given vector field, in an unbounded domain $\Omega$. We prove that, under natural assumptions, this system possesses a locally compact attractor $\mathcal{A}$ in the corresponding phase space. Since the dimension of this attractor is usually infinite, we study its Kolmogorov's $\varepsilon$-entropy and obtain Upper and lower bounds of this entropy.

Moreover, we give a more detailed study of the spatio-temporal chaos generated by the spatially homogeneous $\operatorname{RDS}$ in $\Omega=\mathbb{R}^{n}$. In order to describe this chaos, we introduce an extended $(n+1)$-parametrical semigroup, generated on the attractor by 1-parametrical temporal dynamics and by $n$-parametrical group of spatial shifts (=spatial dynamics). We prove that this extended semigroup has finite topological entropy, in contrast to the case of purely temporal or purely spatial dynamics, where the topological entropy is infinite. We also modify the concept of topological entropy in such a way that the modified one is finite and strictly positive, in particular for purely temporal and for purely spatial dynamics on the attractor.

In order to clarify the nature of the spatial and temporal chaos on the attractor, we introduce a new model dynamical system which is an adaptation of Bernoulli shifts to the case of infinite entropy and construct homeomorphic embeddings of it into the spatial and temporal dynamics on $\mathcal{A}$. As a corollary of the obtained embeddings, we finally prove that every finite dimensional dynamics can be realized (up to a homeomorphism) by restricting the temporal dynamics to the appropriate invariant subset of $\mathcal{A}$.
\end{abstract}

\section{Contents}

\section{Introduction.}

$\S 1$ Functional spaces.

$\S 2$ A priori estimates, existence of solutions, uniqueness.

$\S 3$ The global attractor and upper bounds of its Kolmogorov's $\varepsilon$-entropy.

$\S 4$ Quantitative characteristics for the spatial and temporal dynamics.

$\S 5$ Lower bounds for the $\varepsilon$-entropy and spatial chaos.

1991 Mathematics Subject Classification. 35B40, 35B45.

Key words and phrases. Reaction-diffusion systems, unbounded domains, entropy, spatial and dynamical chaos. 
$\S 6$ The spatial dynamical system in the extended phase space.

$\S 7$ The spatial dynamical system near the exponentially unstable equilibrium.

$\S 8$ Complexity of temporal dynamics in RDS in unbounded domains and temporal chaos.

\section{INTRODUCTION.}

In this paper, the following quasi-linear parabolic boundary problem:

$$
\left\{\begin{array}{l}
\partial_{t} u=a \Delta_{x} u-\left(L, \nabla_{x}\right) u-\lambda_{0} u-f(u)+g, \quad x \in \Omega \\
\left.u\right|_{\partial \Omega}=0,\left.\quad u\right|_{t=0}=u_{0}
\end{array}\right.
$$

in an unbounded domain $\Omega$ (which is assumed to satisfy some natural regularity conditions formulated in $\S 1)$ is considered. Here, $u=\left(u^{1}, \cdots, u^{k}\right)$ is an unknown vector-valued function, $\left(L, \nabla_{x}\right):=\sum_{i=1}^{n} L_{i} \partial_{x_{i}}, f$ and $g$ are given functions, $\lambda_{0}>0$ is a positive constant, $a$ is a given $k \times k$-matrix with a positive symmetric part:

$$
a+a^{*}>0
$$

and $L=L(x) \in C_{b}^{1}(\Omega)$ is a given vector field which satisfies the assumption

$$
\|\operatorname{div} L\|_{L^{\infty}(\Omega)} \leq \lambda_{0} / 2
$$

(We note that, in applications, the vector field $L$ is often a solution of the stationary Navier-Stokes equation and (0.3) is not a great restriction.)

The longtime behavior of solutions of $(0.1)$ is of a great recent interest. It is well known that, under appropriate assumptions on the nonlinear term $f(u)$, this behavior can be described in terms of attractors $\mathcal{A}$ of the corresponding dynamical system generated by (0.1) (see, e.g. [4], [5], [30], [34]). One of the possible choices of these assumptions is the following:

$$
\begin{cases}\text { 1. } & f \in C^{3}\left(\mathbb{R}^{k}, \mathbb{R}^{k}\right) \\ \text { 2. } & f(u) . u \geq-C \\ \text { 3. } & f^{\prime}(u) \geq-K\end{cases}
$$

where $u . v$ denotes a standard inner product in $\mathbb{R}^{k}$ (see, e.g., [4], [19] and [23] for other possibilities). We note that $(0.4)$ is fulfilled for many interesting (from the physical point of view equations) such as Chafee-Infante equation, FitzHughNagumo system, generalized Ginsburg-Landau equations and others.

In the case where the domain $\Omega$ is bounded, the global attractors for $(0.1)$ have been constructed and studied under various assumptions on $f, a$ and $g$ (see [4], [24], [3] and the references therein). In particular, the attractor's existence for (0.1) under assumptions (0.2) and (0.4) has been proved in [40]. It is also proved there that, if the nonlinearity $f$ satisfies the additional growth restriction

$$
|f(u)| \leq C\left(1+|u|^{p}\right), \quad p<1+4 /(n-4),
$$


(for $n \leq 4$ the exponent $p$ may be arbitrarily large), then the corresponding semigroup is differentiable with respect to the initial value $u_{0}$, possesses $L^{\infty}$-bounds and the fractal dimension of the attractor is finite.

In the case where the domain $\Omega$ is unbounded (e.g. $\Omega=\mathbb{R}^{n}$ ), the situation becomes more complicated. In this case, even the choice of the appropriate phase space for $(0.1)$ is a nontrivial problem. Indeed, the phase space $L^{2}(\Omega)$ (as in the case of bounded domains) does not seem to be adequate since a number of natural (from the physical point of view) structures (such as spatially periodic solutions, travelling waves, etc.) do not belong to that space. As a result, the global attractor in $L^{2}(\Omega)$ of $(0.1)$ exists only for very particular cases (see e.g. [5], [7], [17], [29]). That is the reason why, following [21], [33], [39], we will consider equation (0.1) in the spaces

$$
W_{b}^{l, p}(\Omega):=\left\{u_{0} \in D^{\prime}(\Omega):\left\|u_{0}\right\|_{W_{b}^{l, p}}:=\sup _{x_{0} \in \Omega}\left\|u_{0}\right\|_{W^{l, p}\left(\Omega \cap B_{x_{0}}^{1}\right)}<\infty\right\}
$$

with an appropriate choice of exponents $l$ and $p$ (here and below, $B_{x_{0}}^{R}$ denotes the $R$-ball in $\mathbb{R}^{n}$ centered at $x_{0}$ and $W^{l, p}(V)$ is the Sobolev space of functions whose derivatives up to the order $l$ belong to $L^{p}(V)$ ). Roughly speaking, spaces (0.6) consist of all sufficiently regular functions $u_{0}(x)$ which remain bounded as $|x| \rightarrow \infty$ and contain, therefore, all the structures mentioned above.

To the best of our knowledge, the existence of the global attractor of (0.1) in the unbounded domain $\Omega=\mathbb{R}^{n}$ has been firstly established in [1] and [5] (for a scalar case $k=1$ and under the very restrictive growth assumption $p<\min \{4 / n, 2 /(n-$ $2)\})$. These growth restrictions have been removed later in [20] and [29]. The case of systems $(k \geq 2)$ with a scalar diffusion matrix $a$ has been considered in [7], [16], [17], [18], [39]. The case of systems of type (0.1) (without the transport term $\left(L, \nabla_{x} u\right)$ ) with general diffusion matrices (satisfying $\left.(0.2)\right)$ has been considered in [43] under the assumptions on the nonlinear term which are close to (0.4) and (0.5). We mention also that, for the particular cases of equations of the form (0.1), e.g. for complex Ginsburg-Landau equations, more powerful results have been obtained (see [30] and the references therein).

In the present paper (which can be considered as a continuation of our previous paper [43]), we give a comprehensive study of spatio-temporal dynamics generated by problem (0.1) on the corresponding attractor. To this end, we need to consider more general problems of type (0.1) with the nonhomogeneous and nonautonomous boundary condition

$$
\left.u\right|_{\partial \Omega}=u^{0}=u^{0}(t, x), \quad t \in \mathbb{R}_{+}, \quad x \in \partial \Omega
$$

(instead of $\left.u\right|_{\partial \Omega}=0$ ) which requires to impose the following additional regularity assumption to the nonlinear term:

$$
\left|f^{\prime}(u)\right|^{p /(p-1)} \leq C(|f(u)|+|u|+1), \quad \forall u \in \mathbb{R}^{n},
$$

where $p$ is the same as in (0.5). As usual, we first prove that problem (0.1) posseses a unique solution in the appropriate functional class and derive a dissipative estimate for that solution which allows to establish the existence of the global attractor for the semigroup associated with this problem. 
Theorem 1. Let assumptions (0.2)-(0.8) hold, $g \in L_{b}^{q}(\Omega)$, for some $q>n+1$, and the boundary data $u^{0}$ belong to the corresponding space $\Psi_{b}\left(\mathbb{R}_{+} \times \partial \Omega\right.$ ) (which is defined in Section 2). Then, for every $u_{0} \in \Phi_{b}(\Omega):=W_{b}^{2, q}(\Omega) \cap\left\{\left.u_{0}\right|_{\partial \Omega}=u^{0}(0)\right\}$, problem (0.1) possesses a unique solution $u(t) \in \Phi_{b}(\Omega)$, for $t \geq 0$, which satisfies the following estimate:

$$
\|u(t)\|_{\Phi_{b}} \leq Q\left(\left\|u_{0}\right\|_{\Phi_{b}}\right) e^{-\alpha t}+Q\left(\|g\|_{L_{b}^{q}}\right)+Q\left(\left\|u^{0}\right\|_{\Psi_{b}}\right),
$$

where $\alpha$ is a positive constant and $Q$ is an appropriate monotonic function which are independent of $u_{0}$ and, consequently, the solving operators

$$
S_{t}: \Phi_{b}(\Omega) \rightarrow \Phi_{b}(\Omega), \quad t \geq 0, \quad S_{t} u_{0}:=u(t)
$$

are well defined for problem (0.1) and generate a semigroup in the phase space $\Phi_{b}$, if the boundary data $u^{0}=u^{0}(x)$ is independent of $t$.

Moreover, this semigroup possesses a locally compact global attractor $\mathcal{A}$ in the phase space $\Phi_{b}(\Omega)$ (see Section 3 for the details).

We note that, under the assumptions of Theorem 1, the Hausdorff and fractal dimensions of the attractor are usually infinite (see, e.g. [5], [38] and Th. 2 below). That is the reason why the concept of Kolmogorov's $\varepsilon$-entropy is usually exploited in order to obtain some qualitive or/and quantitative information on such attractors $(\varepsilon$-entropy of infinite-dimensional uniform attractors associated with nonautonomous RDE in bounded domains is studied in [9]; the case of autonomous reaction-diffusion equations in $\mathbb{R}^{n}$ is considered in [11] and [38]; the $\varepsilon$-entropy in the case of general unbounded domains are investigated in [18] and [39], for the case of autonomous and nonautonomous RDE, and in [13], [41] and [42], for the case of damped hyperbolic equations).

We recall that, if $K$ is a precompact set in a metric space $M$, then it can be covered (due to the Hausdorff criteria) by a finite number of $\varepsilon$-balls, for every $\varepsilon>0$. Let $N_{\varepsilon}(K, M)$ be the minimal number of such balls. Then, by definition, the Kolmogorov's $\varepsilon$-entropy of $K$ in $M$ is the following number:

$$
\mathbb{H}_{\varepsilon}(K, M):=\ln N_{\varepsilon}(K, M) \text {. }
$$

It is worth to emphasize that, in contrast to the fractal dimension, quantity $(0.10)$ remains finite, for every $\varepsilon>0$ and every precompact set $K$ in $M$.

Moreover, it is proved in [39], [42] and [43] that, for a large class of equations of mathematical physics in unbounded domains (including various types of reaction-diffusion equations, hyperbolic problems, etc.), the $\varepsilon$-entropy of restrictions $\left.\mathcal{A}\right|_{\Omega \cap B_{x_{0}}^{R}}$ of the corresponding attractors $\mathcal{A}$ to bounded domains $\Omega \cap B_{x_{0}}^{R}$ possess the following universal estimate:

$$
\mathbb{H}_{\varepsilon}\left(\left.\mathcal{A}\right|_{\Omega \cap B_{x_{0}}^{R}}, \Phi_{b}\right) \leq C \operatorname{vol}\left(\Omega \cap B_{x_{0}}^{R+K \ln 1 / \varepsilon}\right) \ln \frac{1}{\varepsilon} ; \quad \varepsilon \leq \varepsilon_{0}<1,
$$

where the constants $C, K$ and $\varepsilon_{0}$ depend on the concrete form of the equation, but are independent of $\varepsilon, R$, and $x_{0}$. As shown in Section 3 , this class of equations include, in particular, equations of type (0.1), if the assumptions formulated above are satisfied.

Moreover, following [39] and [43], we also obtain lower bounds for the entropy of restrictions $\left.\mathcal{A}\right|_{B_{x_{0}}^{R}}$ in the case where $\Omega=\mathbb{R}^{n}, g \equiv$ const and $L \equiv$ const and under the natural assumption that (0.1) possesses at least one spatially homogeneous exponentially unstable equilibria point. 
Theorem 2. Let the assumptions of Theorem 1 hold and let $\Omega=\mathbb{R}^{n}, g \equiv 0$, $L \equiv$ const and $f(0)=0$. We also assume that

$$
\sigma\left(a \Delta_{x}-\left(L, \nabla_{x}\right)-f^{\prime}(0)-\lambda_{0}\right) \cap\{z \in \mathbb{C}: \operatorname{Re} z>0\} \neq \varnothing
$$

where $\sigma(L)$ denotes the spectrum of the linear operator $L$. Then, the entropy of the attractor possesses the following estimates:

$$
\mathbb{H}_{\varepsilon}\left(\left.\mathcal{A}\right|_{B_{x_{0}}^{R}}, \Phi_{b}\right) \geq C_{1} R^{n} \ln \frac{1}{\varepsilon}, \quad C_{1}>0, \quad \varepsilon \leq \varepsilon_{0}<1
$$

Moreover, for every $\mu>0$, there exists a constant $C_{\mu}>0$ such that

$$
\mathbb{H}_{\varepsilon}\left(\left.\mathcal{A}\right|_{B_{x_{0}}^{1}}, \Phi_{b}\right) \geq C_{\mu}\left(\ln \frac{1}{\varepsilon}\right)^{n+1-\mu}
$$

We note that, for the particular case $\Omega=\mathbb{R}^{n},(0.11)$ reads

$$
\mathbb{H}_{\varepsilon}\left(\left.\mathcal{A}\right|_{B_{x_{0}}^{R}}, \Phi_{b}\right) \leq C_{2}\left(R+K \ln \frac{1}{\varepsilon}\right)^{n} \ln \frac{1}{\varepsilon}
$$

Therefore, Theorem 2 shows that estimate $(0.11)$ is sharp, at least in the case $\Omega=\mathbb{R}^{n}$. On the other hand, in the case where the domain $\Omega$ is bounded, estimate (0.11) yields

$$
\mathbb{H}_{\varepsilon}(\mathcal{A}, \Phi) \leq C \operatorname{vol}(\Omega) \ln \frac{1}{\varepsilon}
$$

which reflects the well-known heuristic principle that the equations of mathematical physics in bounded domains have the finite fractal dimension (and, moreover, indicates in a right way the dependence of this dimension on the 'size' of $\Omega$ ). Thus, estimate (0.11) may be considered as a natural generalization of this principle to the case of unbounded domains (see also [18] or [42]).

The main part of the paper is devoted to the more detailed study of the spatially homogeneous case of equation $(0.1)\left(\Omega=\mathbb{R}^{n}, g \equiv\right.$ const, $L \equiv$ const $)$. In this case, the attractor $\mathcal{A}$ possesses an additional structure, namely, it is invariant under the group $\left\{T_{h}, h \in \mathbb{R}^{n}\right\}$ of spatial shifts:

$$
T_{h}: \mathcal{A} \rightarrow \mathcal{A}, \quad T_{h} \mathcal{A}=\mathcal{A}, \quad h \in \mathbb{R}^{n}, \quad\left(T_{h} u_{0}\right)(x):=u_{0}(x+h)
$$

and, consequently, it is invariant under the extended $(n+1)$-parametrical semigroup $\mathbb{S}_{(t, h)}$ defined by the following expression:

$$
\mathbb{S}_{(t, h)} u_{0}:=T_{h} S_{t} u_{0}, \quad \mathbb{S}_{(t, h)} \mathcal{A}=\mathcal{A}
$$

In the present paper, we suggest to interpret this semigroup as a dynamical system (with multidimensional 'time') acting in the phase space $\mathcal{A}$. Thus, in order to study the spatio-temporal complexity (and spatio-temporal chaos) of $\mathcal{A}$, we will below investigate the dynamical properties of system $(0.17)$. 
Theorem 3. Let the assumption of Theorem 1 hold and let, in addition, equation (0.1) be spatially homogeneous. Then, the topological entropy (see e.g. [25]) of semigroup (0.17) is finite

$$
h_{\text {top }}\left(\mathcal{A}, \mathbb{S}_{(t, h)}\right)<\infty
$$

and coincides with the topological entropy per unit volume introduced in [12].

Thus, Theorem 3 gives a simple geometrical interpretation for the topological entropy per unit volume.

It is also reasonable to study the dynamical systems, generated by $k$-parametrical subgroups of the extended semigroup $(0.17)$, namely, let $V_{k}$ be an arbitrary $k$ dimensional hyper-plane $V_{k} \subset \mathbb{R}_{t} \times \mathbb{R}_{x}^{n}$ and let

$$
\mathbb{S}_{(t, h)}^{V_{k}}:=\left\{\mathbb{S}_{(t, h)}, t \geq 0,(t, h) \in V_{k}\right\}
$$

be the $k$-parametrical dynamical system which corresponds to the hyper-plane $V_{k}$.

The most natural choices of the plane $V_{k}$ are the following: $1 . k=n, V_{n}=\mathbb{R}_{x}^{n}$, then $\mathbb{S}_{(t, h)}^{V_{n}}:=T_{h}$, which corresponds to the purely spatial dynamics on the attractor and 2. $k=1, V_{1}=\mathbb{R}_{t}$, then $\mathbb{S}_{(t, h)}^{V_{1}}:=S_{t}$, which corresponds to the purely temporal evolution restricted to the attractor; although intermediate choices of $V_{k}$, which describe the interaction between the spatial and temporal modes are of independent interest.

We note that, in contrast to the case of $k=n+1$, the topological entropy for dynamical systems (0.19) may be infinite (and is usually infinite as shown below) if $k<n+1$. Consequently, new quantitative characteristics of the complexity are required for these cases. We suggest to use in this situation the modified topological entropies which differ from the classical one by presence of the factor $(\ln 1 / \varepsilon)^{k-n-1}$ (for $k$-dimensional subgroups) in their definition (see Section 4). For instance, if $V_{n}=\mathbb{R}_{x}^{n}$, then

$$
\widehat{h}_{s p}(\mathcal{A}):=\widehat{h}_{n}^{V_{n}}(\mathcal{A}):=\limsup _{\varepsilon \rightarrow 0}\left(\ln \frac{1}{\varepsilon}\right)^{-1} \lim _{R \rightarrow \infty} \frac{1}{R^{n}} \mathbb{H}_{\varepsilon}\left(\mathcal{A}, L^{\infty}\left([0, R]^{n}\right)\right)
$$

and, for $V_{1}=\mathbb{R}_{t}$, we have

$$
\widehat{h}_{t}(\mathcal{A}):=\widehat{h}_{1}^{V_{1}}(\mathcal{A}):=\limsup _{\varepsilon \rightarrow 0}\left(\ln \frac{1}{\varepsilon}\right)^{-n} \lim _{T \rightarrow \infty} \frac{1}{T} \mathbb{H}_{\varepsilon}\left(\mathcal{K}, L^{\infty}\left([0, T], L_{e^{-|x|}}^{\infty}\left(\mathbb{R}^{n}\right)\right)\right),
$$

where $\mathcal{K}$ denotes the set of all the solutions $u(t), t \in \mathbb{R}$, of $(0.1)$ which belong to $L^{\infty}\left(\mathbb{R}, \Phi_{b}\right)$ (see Section 3 ) and $L_{e^{-|x|}}^{\infty}(\Omega)$ is a weighted space (see Section 1 ).

The following theorem, which can be treated as a generalization of the wellknown relation between the fractal dimension and topological entropy (see e.g. [25]) describes the relations between $\widehat{h}_{k}^{V_{k}}(\mathcal{A})$ computed for different hyperplanes $V_{k}$.

Theorem 4. Let the assumptions of Theorem 3 hold. Then, for every $k, 0 \leq k \leq$ $n+1$ and for every hyperplane $V_{k}$, the corresponding (modified) topological entropy is finite:

$$
\widehat{h}_{k}^{V_{k}}(\mathcal{A})<\infty .
$$


Moreover, if $V_{k} \subset V_{l}(k<l)$, then

$$
\widehat{h}_{l}^{V_{l}}(\mathcal{A}) \leq K^{l-k} \widehat{h}_{k}^{V_{k}}(\mathcal{A})
$$

where the constant $K>0$ depends only on the equation.

Thus, it folows from (0.21) that, if the topological entropy of extended semigroup (0.17) is strictly positive, then the (modified) topological entropy of dynamical system $(0.19)$ is also strictly positive for every hyperplane $V_{k}$. Unfortunately, the problem of obtaining the lower bounds for quantity (0.17) for more or less concrete equations of mathematical physics is extremely difficult, even in the case $n=0$ (which corresponds to the ODE of type (0.1), see [25] and the references therein), although several examples of equations of type (0.1), for which the corresponding spatio-temporal topological entropy is strictly positive, has been recently constructed (see [44]).

In contrast to that, the case of the dynamics associated with $n$-dimensional hyperplanes $V_{n} \subset \mathbb{R}_{t} \times \mathbb{R}_{x}^{n}$ is simpler and we give below some natural (and effective) sufficient conditions which allow to verify that the corresponding modified topological entropies are strictly positive for a large class of equations of mathematical physics.

We start with the case $V_{n}=\mathbb{R}_{x}^{n}$, which describes the purely spatial dynamics. The phenomena of spatial complexity and spatial chaos has been studied, e.g. in [2], [6], [14], [15], [43] (see also the references therein) for several particular cases of equation (0.1). In our case, estimate (0.13), obviously, implies that the modified topological entropy of spatial dynamical system $(0.16)\left(V_{n}:=\mathbb{R}_{x}^{n}\right)$ is strictly positive

$$
\widehat{h}_{s p}(\mathcal{A})>C>0
$$

and, consequently, the classical (non modified) topological entropy of that semigroup is infinite, if the assumptions of Theorem 2 are satisfied. Thus, the dynamical behavior of $(0.16)$ is extremely chaotic. We also note that, in contrast to the case of dynamical chaos, generated by ODE or by PDE in bounded domains, the symbolic dynamics (Bernoulli shifts with finite number of symbols, see e.g. [25]) is not an adequate model example for understanding the nature of the spatial chaos in $(0.16)$, since the topological entropy of such symbolic dynamics is finite. That is the reason why (following [43]), we use another model dynamical system which generalizes the classical Bernoulli shifts and is adopted to the case of infinite topological entropy. Namely, let $\mathbb{D}$ be a unit disc in $\mathbb{C}$ and let $\mathcal{M}:=\mathbb{D}^{\mathbb{Z}^{n}}$ endowed by the Tikhonov's topology. Then, a discrete dynamical system $\mathcal{T}_{h}$ with multidimensional 'time' $h \in \mathbb{Z}^{n}$ on $\mathcal{M}$ can be defined in the following natural way:

$$
\mathcal{T}_{h} v(l):=v(h+l), \quad h, l \in \mathbb{Z}^{n}, \quad v \in \mathcal{M} .
$$

(We recall that, as usual, $\mathcal{M}$ is interpreted as a space of functions $v: \mathbb{Z}^{n} \rightarrow \mathbb{D}$ ).

Applying a general scheme of investigating the spatial complexity of the attractors of equations of mathematical physics developed in [39], [42] and [43] to equation (0.1), we derive (in Section 5) the following result which clarifies the nature of the spatial chaos in (0.16). 
Theorem 5. Let the assumptions of Theorem 2 hold. Then, there exists a positive number $\sigma>0$, a closed subset $K \subset \mathcal{A}$ and a homeomorphism $\tau: \mathcal{M} \rightarrow K$ such that

$$
T_{\sigma h} K=K \quad \text { and } \quad T_{\sigma h} \tau(v)=\tau\left(\mathcal{T}_{h} v\right), \quad \forall h \in \mathbb{Z}^{n}, v \in \mathcal{M}
$$

Moreover, this homeomorphism is Lipschitz continuous under the appropriate choice of metrics on $\mathcal{A}$ and $\mathcal{M}$ and preserves the modified topological entropy:

$$
0<\widehat{h}_{s p}(\mathcal{M})=\widehat{h}_{s p}(K) \leq \widehat{h}_{s p}(\mathcal{A})<\infty .
$$

As an immediate corollary of this result, we obtain the fact that every finite dimensional dynamics can be realized (up to a homeomorphism) by restricting spatial dynamical system (0.16) to appropriate closed subsets of $\mathcal{A}$.

Corollary 1. Let the assumptions of Theorem 5 hold, let $K \subset \mathbb{R}^{N}$ be an arbitray compact set in $\mathbb{R}^{N}, N \in \mathbb{N}$, and let $F_{1}, \cdots, F_{n}: K \rightarrow K$ be arbitrary pairwise commutative homeomorphisms, i.e.

$$
F_{i} \circ F_{j}=F_{j} \circ F_{i}, \quad i, j \in\{1, \cdots, n\} .
$$

Then, there exist a positive number $\gamma=\gamma(N)<0$ and a homeomorphism

$$
\widehat{\tau}: K \rightarrow \widehat{\tau}(K) \subset \mathcal{A},
$$

such that

$$
T_{\gamma l} \widehat{\tau}(k)=\widehat{\tau}\left(F_{1}^{l_{1}} \circ \cdots \circ F_{n}^{l_{n}} k\right), \quad k \in K, \quad l \in \mathbb{Z}^{n},
$$

where $F_{i}^{l_{i}}$ denotes the $l_{i}$-th iteration of the map $F_{i}$.

This result confirms, from the alternative point of view, that spatial dynamics (0.16) is indeed extremely chaotic.

The main task of the rest of the paper is to obtain the analogue of Theorem 5 in the case where $V_{n}$ contains a temporal direction and to include, thus, the temporal dynamics into consideration. We note that the general scheme of investigating the phenomena of spatial chaos mentioned above is based on the technique of infinite dimensional unstable manifolds and gives no information on the temporal evolution (at least in a direct way). Nevertheless, we suggest below a trick which allows to obtain such an information based on this scheme. The main idea of this trick is to construct a new auxiliary dynamical system the attractor of which coincides (in a sense) with the attractor of the initial system (0.1) and such that the direction $t$ is occured to be 'spatial' for this dynamical system. Applying the scheme of studying the spatial complexity to this auxiliary system, we obtain simultaneously the description of the temporal evolution for the initial problem (since the direction $t$ is 'spatial' for that auxiliary system!).

In order to construct this auxiliary dynamical system, we assume that the vector field $L$ has the form $L:=L(1,0, \cdots, 0$ ), where $L>0$ (a general case can be easily reduced to this one by an appropriate rotation) and consider the following boundary value problem:

$$
\left\{\begin{array}{l}
a\left(\partial_{x_{1}}^{2} u+\Delta_{x^{\prime}} u\right)-L \partial_{x_{1}} u-\lambda_{0} u-f(u)=\partial_{t} u, \\
\left.u\right|_{x_{1}=0}=u^{0}, t \in \mathbb{R}, x_{1} \in \mathbb{R}_{+}, x^{\prime} \in \mathbb{R}^{n-1},
\end{array}\right.
$$


where $x=\left(x_{1}, x^{\prime}\right)$. Boundary value problem (0.26) can be formally interpreted as an evolution equation with respect to $x_{1}$ and 'spatial' directions $t, x^{\prime}$. Moreover, it is proved (in Section 6) that this boundary value problem defines indeed a differentiable and dissipative dynamical system on the appropriate trace space $\Psi_{b}$, if $L>0$ is large enough (see Theorem 6.1 for the rigorous statement). Then, on the one hand, the sets of all complete bounded solutions of equations (0.1) and (0.26) which are defined for all $(t, x) \in \mathbb{R}^{n+1}$ coincide and, on the other hand, the hyperplane $V_{n}:=\operatorname{span}\left\{t, x_{2}, \cdots, x_{n}\right\}$ corresponds now to the spatial dynamics for this system. Applying the above general scheme to that auxiliary dynamical system, we obtain (in Section 8) the following result.

Theorem 6. Let the assumptions of Theorem 2 hold and let, in addition, the vector field $L$ have the form $L=L(1,0, \cdots, 0)$, where $L>0$ is large enough (see condition (6.6)). Then, the modified topological entropy of dynamical system (0.19) where $V_{n}:=\operatorname{span}\left\{t, x^{\prime}\right\}$ is strictly positive

$$
\widehat{h}_{n}^{V_{n}}(\mathcal{A})>C>0
$$

and, consequently, thanks to Theorem 4, the modified temporal topological entropy is also strictly positive

$$
\widehat{h}_{t}(\mathcal{A})>C_{1}>0
$$

and the classical (nonmodified) one equals infinity.

Thus, the temporal dynamics is also exteremely chaotic under the assumptions of Theorem 6. Moreover, analogously to Theorem 5, we obtain the following embedding.

Theorem 7. Let the assumptions of Theorem 6 hold and let, in addition, the diffusion matrix satisfy the technical assumption $a a^{*}=a^{*} a$. Then, there exist $a$ number $\alpha>0$ and a homeomorphic embedding

$$
\widehat{\tau}: \mathcal{M} \rightarrow \mathcal{A}
$$

such that

$$
S_{\alpha l} \widehat{\tau}\left(v_{0}\right)=\widehat{\tau}\left(\mathcal{T}_{l}^{x_{1}} v_{0}\right), \quad T_{\alpha l}^{x_{i}} \widehat{\tau}\left(v_{0}\right)=\widehat{\tau}\left(\mathcal{T}_{l}^{x_{i}} v_{0}\right), i=2, \cdots, n,
$$

for every $l \in \mathbb{Z}$ and every $v_{0} \in \mathcal{M}$. Moreover,

$$
\widehat{h}_{n}^{V_{n}}(\widehat{\tau}(\mathcal{M}))>0
$$

where $V_{n}:=\operatorname{span}\left\{t, x_{2}, \cdots, x_{n}\right\}$.

As in the case of spatial dynamics, embedding (0.29) allows to prove that every finite dimensional dynamics can be realized (up to a homeomorphism) by restricting the temporal evolution semigroup $S_{t}$ to the appropriate invariant subset of the attractor. 
Corollary 2. Let the assumptions of Theorem 7 hold, $K \subset \mathbb{R}^{N}$ be an arbitrary compact set in $\mathbb{R}^{N}, N \in \mathbb{N}$, and $F_{1}, \cdots, F_{n}: K \rightarrow K$ be arbitrary pairwise commutative homeomorphisms, i.e.

$$
F_{i} \circ F_{j}=F_{j} \circ F_{i}, \quad i, j \in\{1, \cdots, n\} .
$$

Then, there exist a positive number $\gamma=\gamma(N)>0$ and a homeomorphism

$$
\tilde{\tau}: K \rightarrow \tilde{\tau}(K) \subset \mathcal{A}
$$

such that

$$
S_{\gamma l_{1}} \circ T_{\gamma l_{2}}^{x_{2}} \cdots \circ T_{\gamma l_{n}}^{x_{n}} \tilde{\tau}(k)=\tilde{\tau}\left(F_{1}^{l_{1}} \circ \cdots F_{n}^{l_{n}} k\right), \quad k \in K, \quad l \in \mathbb{Z}^{n},
$$

where $F_{i}^{l_{i}}$ denotes the $l_{i}$-th iteration of the map $F_{i}$.

We illustrate the obtained results on the simplest example of one dimensional Chafee-Infante equation pertubed by the transport term

$$
\partial_{t} u=\partial_{x}^{2} u-L \partial_{x} u+u-u^{3}, \quad x \in \mathbb{R}^{1} .
$$

It can be easily verified that all the assumptions of Theorem 7 are satisfied for $(0.34)$, if $L>2$. Consequently, in this case, (0.34) generates an extremely complicated temporal dynamics. In particular, this equation possesses a noncountable number of essentially different time periodic solutions (which are parametrized by the periodic orbits of (generalized) Bernoulli shifts $\left.\left(\mathcal{M}, \mathcal{T}_{l}\right)\right)$. We also note that, for $L=0$, (0.34) generates the so-called extended gradient system and, consequently (see [22]), this system does not possess any time periodic solution (any uniformly reccursive solution and so on). Thus, simplest model (0.34) gives a good example illustrating the influence of transport terms to the reaction-diffusion dynamics.

The paper is organized as follows. Definitions of functional spaces, which are of fundamental significance for our study equation (0.1), and their simple properties are given in Section 1. Various a priori estimates for the solutions of (0.1) are obtained in Section 2. Moreover, based on these estimates, we verify the existence of a solution, its uniqueness and derive some estimates for the differences of solutions which will be essentially used later. The existence of a global attractor $\mathcal{A}$ for system (0.1) is verified in Section 3. Moreover, the upper bounds of its Kolmogorov's $\varepsilon$ entropy are obtained here. Quantitative characteristics for the $(n+1)$-parametrical extended dynamical system and for its $k$-parametrical subgroups are investigated in Section 4. In particular, the proof of Theorem 4 is given here. In Section 5, we deduce the lower bounds of $\varepsilon$-entropy for equation (0.1) and give a topological description of the phenomena of spatial complexity and spatial chaotisity (in particular, Theorems 2 and 5 are proved here). General analytic properties of auxiliary spatially dynamical system (0.26) (such as existence of solutions, their uniqueness, smoothness and so on) are obtained in Section 6. The behaviour of the auxiliary spatial dynamical system near the exponentially unstable equilibria point is investigated in Section 7. The main result of this Section is the existence of an infinite dimensional unstable manifold of this dynamical system. The topological description of temporal complexity of the dynamics generated by (0.1) (Theorems 6 and 7 ) is obtained in Section 8 based on the results of Section 7.

Acknowledgements. The author has greatly benefited from helpful comments of M.Efendiev, A.Mielke, D.Turaev and M.Vishik. 


\section{$\S 1$ Functional spaces.}

In this Section, we introduce several classes of Sobolev spaces in unbounded domains and recall shortly some of their properties which will be essentially used below. For the detailed study of these spaces, see [17] and [39].

Definition 1.1. A function $\phi \in C_{l o c}\left(\mathbb{R}^{n}\right)$ is a weight function with (exponential) growth rate $\mu \geq 0$ if the condition

$$
\phi(x+y) \leq C_{\phi} e^{\mu|x|} \phi(y), \phi(x)>0
$$

is satisfied, for every $x, y \in \mathbb{R}^{n}$. Analogously, a function $\phi \in C_{l o c}\left(\mathbb{R}^{n}\right)$ is a weight function with polynomial growth rate $\mu$ if the following inequality is valid, for every $x, y \in \mathbb{R}^{n}:$

$$
\phi(x+y) \leq C_{\phi}\left(\left(1+\left|y_{1}\right|^{2}\right)\left(1+\left|y_{2}\right|^{2}\right) \cdots\left(1+\left|y_{n}\right|^{2}\right)\right)^{\mu / 2} \phi(x), \quad \phi(x)>0 .
$$

Remark 1.1. Obviously, every weight function which satisfies (1.2), for some $\mu \geq$ 0 , satisfies automatically (1.1), for every $\mu>0$. Moreover, it is not difficult to deduce from (1.1) that

$$
\phi(x+y) \geq C_{\phi}^{-1} e^{-\mu|x|} \phi(y)
$$

is also satisfied, for every $x, y \in \mathbb{R}^{n}$. Estimates (1.1) and (1.3) imply, in particular, that

$$
C_{\phi}^{-1} e^{-\mu R} \phi(x) \leq \sup _{\left|x_{0}\right| \leq R} \phi\left(x-x_{0}\right) \leq C_{\phi} e^{\mu R} \phi(x) .
$$

The following examples of weight functions are of fundamental significance for our purposes:

$$
\phi_{\varepsilon, x_{0}}(x)=e^{-\varepsilon\left|x-x_{0}\right|}, \varepsilon \in \mathbb{R}, x_{0} \in \mathbb{R}^{n} .
$$

Obviously, these weights have growth rate $|\varepsilon|$ and satisfy (1.1) uniformly with respect to $x_{0} \in \mathbb{R}^{n}$ (i.e., the constant $C_{\phi_{\varepsilon}, x_{0}}$ in (1.1) is independent of $x_{0}$ ).

Analogously, the model example of a weight function with polynomial growth rate is the following:

$$
\varphi_{\mu, x_{0}}(x)=\left(\left(1+\left|x^{1}-x_{0}^{1}\right|^{2}\right) \cdots\left(1+\left|x^{n}-x_{0}^{n}\right|^{2}\right)\right)^{\mu / 2}, \quad x_{0} \in \mathbb{R}^{n}, \quad \mu \in \mathbb{R} .
$$

Obviously, weights (1.6) have polynomial growth rate $|\mu|$ and also satisfy (1.2) uniformly with respect to $x_{0} \in \mathbb{R}$.

Definition 1.2. Let $\Omega \subset \mathbb{R}^{n}$ be some (unbounded) domain in $\mathbb{R}^{n}$ and let $\phi$ be a weight function with growth rate $\mu$. We set

$$
L_{\phi}^{p}(\Omega)=\left\{u \in D^{\prime}(\Omega):\|u, \Omega\|_{\phi, 0, p}^{p} \equiv \int_{\Omega} \phi(x)|u(x)|^{p} d x<\infty\right\} .
$$

Analogously, the weighted Sobolev space $W_{\phi}^{l, p}(\Omega), l \in \mathbb{N}$, is defined as the space of distributions whose derivatives up to the order $l$ belong to $L_{\phi}^{p}(\Omega)$.

In order to simplify the notations, we will write below $W_{\{\varepsilon\}}^{s, p}$ instead of $W_{e^{-\varepsilon|x|}}^{s, p}$.

We also define another class of weighted Sobolev spaces as follows:

$$
W_{b, \phi}^{l, p}(\Omega)=\left\{u \in D^{\prime}(\Omega):\|u, \Omega\|_{b, \phi, l, p}^{p}=\sup _{x_{0} \in \Omega} \phi\left(x_{0}\right)\left\|u, \Omega \cap B_{x_{0}}^{1}\right\|_{l, p}^{p}<\infty\right\} .
$$

Here and below, we denote by $B_{x_{0}}^{R}$ the ball in $\mathbb{R}^{n}$ of radius $R$, centered at $x_{0}$ and $\|u, V\|_{l, p}$ stands for $\|u\|_{W^{l, p}(V)}$.

We will write $W_{b}^{l, p}$ instead of $W_{b, 1}^{l, p}$. 


\section{Proposition 1.1.}

1. Let $u$ belong to $L_{\phi}^{p}(\Omega)$, where $\phi$ is a weight function with growth rate $\mu$. Then, for any $1 \leq q \leq \infty$, the following estimate is valid:

$$
\left(\int_{\Omega} \phi\left(x_{0}\right)^{q}\left(\int_{\Omega} e^{-\varepsilon\left|x-x_{0}\right|}|u(x)|^{p} d x\right)^{q} d x_{0}\right)^{1 / q} \leq C \int_{\Omega} \phi(x)|u(x)|^{p} d x,
$$

for every $\varepsilon>\mu$, where the constant $C$ depends only on $\varepsilon, \mu$ and the constant $C_{\phi}$ from (1.1) (and is independent of $\Omega$ ).

2. Let $u$ belong to $L_{\phi}^{\infty}(\Omega)$. Then, the following analogue of estimate (1.7) is valid:

$$
\sup _{x_{0} \in \Omega}\left\{\phi\left(x_{0}\right) \sup _{x \in \Omega}\left\{e^{-\varepsilon\left|x-x_{0}\right|}|u(x)|\right\}\right\} \leq C \sup _{x \in \Omega}\{\phi(x)|u(x)|\} .
$$

The proof of this proposition can be found in [17] or [39].

In order to study nonlinear RDS (0.1), we need some regularity assumptions on the domain $\Omega \subset \mathbb{R}^{n}$, which are assumed to be valid throughout of the paper.

We assume that there exists a positive number $R_{0}>0$ such that, for every point $x_{0} \in \Omega$, there exists a smooth domain $V_{x_{0}} \subset \Omega$ such that

$$
B_{x_{0}}^{R_{0}} \cap \Omega \subset V_{x_{0}} \subset B_{x_{0}}^{R_{0}+1} \cap \Omega \text {. }
$$

Moreover, we also assume that there exists a diffeomorphism $\theta_{x_{0}}: B_{0}^{2} \rightarrow B_{x_{0}}^{R_{0}+2}$ such that $\theta_{x_{0}}(x)=x_{0}+p_{x_{0}}(x), \theta_{x_{0}}\left(B_{0}^{1}\right)=V_{x_{0}}$ and

$$
\left\|p_{x_{0}}\right\|_{C^{N}}+\left\|p_{x_{0}}^{-1}\right\|_{C^{N}} \leq K
$$

where the constant $K$ is independent of $x_{0} \in \Omega$ and $N$ is large enough. For simplicity, we assume from now on that (1.9) and (1.10) hold for $R_{0}=2$.

We note that, in case $\Omega$ is bounded, conditions (1.9) and (1.10) are equivalent to the following: the boundary $\partial \Omega$ is a smooth manifold. Now, for unbounded domains, the sole smoothness of the boundary is not sufficient to obtain the regular structure of $\Omega$ as $|x| \rightarrow \infty$, since some uniform with respect to $x_{0} \in \Omega$ smoothness conditions are required. It is however more convenient to formulate these conditions in the form (1.9) and (1.10).

Proposition 1.2. Let the domain $\Omega$ satisfy conditions (1.9) and (1.10), the weight function $\phi$ satisfy condition (1.1) and $R$ be some positive number. Then, the following estimates are valid:

$$
C_{2} \int_{\Omega} \phi(x)|u(x)|^{p} d x \leq \int_{\Omega} \phi\left(x_{0}\right) \int_{\Omega \cap B_{x_{0}}^{R}}|u(x)|^{p} d x d x_{0} \leq C_{1} \int_{\Omega} \phi(x)|u(x)|^{p} d x .
$$

The proof of this proposition is given in [17] or [39].

Corollary 1.1. Let (1.9) and (1.10) hold. Then, the following norm is equivalent to the usual norm in in $W_{\phi}^{l, p}(\Omega)$ :

$$
\|u, \Omega\|_{\phi, l, p}=\left(\int_{\Omega} \phi\left(x_{0}\right)\left\|u, \Omega \cap B_{x_{0}}^{R}\right\|_{l, p}^{p} d x_{0}\right)^{1 / p} .
$$


In particular, norms (1.12) are equivalent, for $R \in \mathbb{R}_{+}$.

To study equation (0.1), we also need weighted Sobolev spaces of fractional order $s \in \mathbb{R}_{+}$(and not for $s \in \mathbb{Z}$ only). We first recall (see [35] for details) that, if $V$ is a bounded domain, a classical norm in the space $W^{s, p}(V), s=[s]+l, 0<l<1$, $[s] \in \mathbb{Z}_{+}$, can be defined by

$$
\|u, V\|_{s, p}^{p}=\|u, V\|_{[s], p}^{p}+\sum_{|\alpha|=[s]} \int_{x \in V} \int_{y \in V} \frac{\left|D^{\alpha} u(x)-D^{\alpha} u(y)\right|^{p}}{|x-y|^{n+l p}} d x d y .
$$

It is not difficult to prove, arguing as in Proposition 1.2 (see [17]) and using this representation, that, for any bounded domain $V$ with a sufficiently smooth boundary

$$
C_{1}\|u, V\|_{s, p}^{p} \leq \int_{x_{0} \in V}\left\|u, V \cap B_{x_{0}}^{R}\right\|_{s, p}^{p} d x_{0} \leq C_{2}\|u, V\|_{s, p}^{p}
$$

This justifies the following definition.

Definition 1.3. We define the space $W_{\phi}^{s, p}(\Omega)$, for $s \in \mathbb{R}_{+}$, as the space of distributions whose norm (1.12) is finite.

It is not difficult to check that these norms are also equivalent for different $R>0$.

In order to consider problem (0.1) with nonhomogeneous boundary conditions, we need the following proposition.

Proposition 1.3. Let the domain $\Omega$ satisfy assumptions (1.9) and (1.10).Then, for every $\varepsilon>0$ and $x_{0} \in \mathbb{R}^{n}$, the following estimate is valid:

$$
\int_{\partial \Omega} e^{-\varepsilon\left|x-x_{0}\right|} d S \leq C_{\varepsilon}
$$

where the constant $C_{\varepsilon}$ is independent of $x_{0}$.

Proof. Let $\vec{n}(x) \in W_{b}^{1, \infty}(\Omega)$ be an arbitrary extention of the normal vector field from $\partial \Omega$ inside of the domain $\Omega$ (the existence of such an extention is guaranteed by conditions (1.9) and (1.10)). Then, due to the Gauss' formula

$$
\int_{\partial \Omega} \phi_{\varepsilon, x_{0}} d S=\int_{\Omega} \operatorname{div}\left(\phi_{\varepsilon, x_{0}} \vec{n}(x)\right) d x \leq C\|\vec{n}, \Omega\|_{b, 1, \infty}\left\|\phi_{\varepsilon, x_{0}}, \Omega\right\|_{1,1} \leq C_{\varepsilon}
$$

and Proposition 1.3 is proved

Corollary 1.2. Let the assumptions of Proposition 1.3 hold. Then,

$$
\int_{\partial \Omega} e^{-\varepsilon\left|x-x_{0}\right|} d S \leq C_{\varepsilon} e^{-\varepsilon / 2 \operatorname{dist}\left(x_{0}, \partial \Omega\right)}
$$

where $\operatorname{dist}\left(x_{0}, \partial \Omega\right)$ denotes the distanse from the point $x_{0}$ to the boundary $\partial \Omega$.

Indeed,

$$
\int_{\partial \Omega} e^{-\varepsilon\left|x-x_{0}\right|} d S \leq \sup _{x \in \partial \Omega}\left\{e^{-\varepsilon / 2\left|x-x_{0}\right|}\right\} \int_{\partial \Omega} e^{-\varepsilon / 2\left|x-x_{0}\right|} d S \leq C_{\varepsilon} e^{-\varepsilon / 2 \operatorname{dist}\left(x_{0}, \partial \Omega\right)} .
$$


Definition 1.4. Analogously to Definition 1.2, we define the weighted Sobolev spaces of functions defined on the boundary $\partial \Omega$. For instance, the weighted space $W_{\phi}^{l, p}(\partial \Omega)$ is determined by the following norm:

$$
\left\|u_{0}, \partial \Omega\right\|_{\phi, l, p}^{p}:=\int_{\partial \Omega} \phi(s)\left\|u_{0}, \partial \Omega \cap B_{s}^{1}\right\|_{l, p}^{p} d S
$$

The spaces $W_{b, \phi}^{l, p}(\partial \Omega)$ are defined analogously.

We now note that the weight functions (1.5) satisfy the conditions (1.1) uniformly with respect to $x_{0} \in \mathbb{R}^{n}$, consequently, all the estimates obtained above for the arbitrary weights will be valid for family (1.5) uniformly with respect to $x_{0} \in \mathbb{R}^{n}$. Since these estimates are of fundamental significance for what follows, we write them explicitly in the following propositions.

Proposition 1.4. Let $u$ belong to $L_{\{\delta\}}^{p}(\Omega)$, for $0<\delta<\varepsilon$. Then, the following estimate holds uniformly with respect to $y \in \mathbb{R}^{n}$ :

$$
\begin{aligned}
\left(\int_{\Omega} e^{-q \delta\left|x_{0}-y\right|}\left(\int_{\Omega} e^{-\varepsilon\left|x-x_{0}\right|}|u(x)|^{p} d x\right)^{q}\right. & \left.d x_{0}\right)^{1 / q} \leq \\
& \leq C_{\varepsilon, q} \int_{\Omega} e^{-\delta|x-y|}|u(x)|^{p} d x .
\end{aligned}
$$

Moreover, if $u \in L_{\{\delta\}}^{\infty}(\Omega), \delta<\varepsilon$, then

$$
\sup _{x_{0} \in \Omega}\left\{e^{-\delta\left|x_{0}-y\right|} \sup _{x \in \Omega}\left\{e^{-\varepsilon\left|x-x_{0}\right|}|u(x)|\right\}\right\} \leq C_{\varepsilon, \delta} \sup _{x \in \Omega}\left\{e^{-\delta|x-y|}|u(x)|\right\} \text {. }
$$

Proposition 1.5. Let $u$ belong to $W_{b, \phi}^{l, p}(\Omega)$ and $\phi$ be a weight function with growth rate $\mu<\varepsilon$. Then

$$
\begin{aligned}
& C_{1}\|u, \Omega\|_{b, \phi, l, p}^{p} \leq \\
& \quad \leq \sup _{x_{0} \in \Omega}\left\{\phi\left(x_{0}\right) \int_{x \in \Omega} e^{-\varepsilon\left|x-x_{0}\right|}\left\|u, \Omega \cap B_{x}^{1}\right\|_{l, p}^{p} d x\right\} \leq C_{2}\|u, \Omega\|_{b, \phi, l, p}^{p} .
\end{aligned}
$$

For the proof of this corollary, see [39].

The following analogue of Proposition 1.5 is valid for polinomial weights (1.6).

Proposition 1.6. Let $\phi$ be a weight function with polynomial growth rate $\mu<N$. Then, the following estimate is valid:

$$
\begin{aligned}
& C_{1} \sup _{x_{0} \in \Omega} \phi\left(x_{0}\right) u\left(x_{0}\right) \leq \\
& \leq \sup _{x \in \Omega}\left\{\phi(x) \sup _{y \in \Omega}\left(\left(1+\left|x_{1}-y_{1}\right|^{2}\right) \cdots\left(1+\left|x_{n}-y_{n}\right|^{2}\right)\right)^{-N / 2} u(y)\right\} \leq \\
& \leq C_{2} \sup _{x_{0} \in \Omega} \phi\left(x_{0}\right) u\left(x_{0}\right) .
\end{aligned}
$$

The proof of this proposition is completely analogous to that of Proposition 1.5 (see e.g. [43]). 
In conclusion of this Section, we inroduce the anysotropic Sobolev spaces of functions defined on $\mathbb{R}_{+} \times \Omega$ or $\mathbb{R}_{+} \times \partial \Omega$.

Definition 1.5. We denote by $W^{\left(l_{1}, l_{2}\right), q}([T, T+1] \times \Omega)$ the classical SobolevSlobodetskij space of functions which have $t$-derivatives up to the order $l_{1}$ and $x$-derivatives up to the order $l_{2}$ belonging to $L^{q}$ (see e.g. [28]). We recall that, for integer $l_{i} \geq 0$, the norm in this space is defined by

$$
\begin{aligned}
& \|u\|_{W^{\left(l_{1}, l_{2}\right), q}([T, T+1] \times \Omega)}^{q}:=\left\|\partial_{t}^{l_{1}} u\right\|_{L_{b}^{q}([T, T+1] \times \Omega)}^{q}+ \\
& +\left\|D_{x}^{l_{2}} u\right\|_{L_{b}^{q}([T, T+1] \times \Omega)}^{q}+\|u\|_{L_{b}^{q}([T, T+1] \times \Omega)}^{q},
\end{aligned}
$$

where $D_{x}^{l_{2}}$ denotes a collection of all $x$-derivatives of the order $l_{2}$, and, for the noninteger $l_{i}$, can be defined by the interpolation, analogously to (1.13) (see [28] or [35]).

We now define, analogously to Definition 1.2 , the spaces $W_{b}^{\left(l_{1}, l_{2}\right), q}\left(\mathbb{R}_{+} \times \Omega\right)$, $W_{b}^{\left(l_{1}, l_{2}\right), q}\left(\mathbb{R}_{+} \times \partial \Omega\right)$, and the corresponding spaces of functions on $\mathbb{R} \times \Omega$. For instance, the norm in the space $W_{b}^{\left(l_{1}, l_{2}\right), q}\left(\mathbb{R}_{+} \times \partial \Omega\right)$ is defined by the following expression:

$$
\|u\|_{W_{b}^{\left(l_{1}, l_{2}\right), q}\left(\mathbb{R}_{+} \times \partial \Omega\right)}:=\sup _{\substack{T \in \mathbb{R}_{+}, x_{0} \in \partial \Omega}}\|u\|_{W^{\left(l_{1}, l_{2}\right), q\left([T, T+1] \times\left(\partial \Omega \cap B_{x_{0}}^{1}\right)\right.}} .
$$

Moreover, let $\phi=\phi(t, x)$ be a weight function of variables $(t, x)$ with exponential growth rate $\mu$ (see Definition 1.1). Then, we define the spaces $W_{\phi}^{\left(l_{1}, l_{2}\right), q}\left(\mathbb{R}_{+} \times \Omega\right.$ ) and $W_{\phi}^{\left(l_{1}, l_{2}\right), q}\left(\mathbb{R}_{+} \times \partial \Omega\right)$ in a standard way. For instance,

$$
\|u\|_{W_{\phi}^{\left(l_{1}, l_{2}\right), q}\left(\mathbb{R}_{+} \times \partial \Omega\right)}^{q}:=\int_{(T, s) \in \mathbb{R}_{+} \times \partial \Omega} \phi(T, s)\|u\|_{W^{\left(l_{1}, l_{2}\right), q\left([T, T+1] \times\left(\partial \Omega \cap B_{s}^{1}\right)\right.}}^{q} d S d T .
$$

$\S 2$ A PRiori estimates, EXistence of solutions, Uniqueness.

In this Section, we derive several a priori estimates for the solutions of reactiondiffusion system (0.1) with nonhomogeneous boundary conditions

$$
\left\{\begin{array}{l}
\partial_{t} u=a \Delta_{x} u-\left(L(x), \nabla_{x}\right) u-\lambda_{0} u-f(u)+g(x), \quad x \in \Omega, \\
\left.u\right|_{\partial \Omega}=u^{0}(t, x),\left.\quad u\right|_{t=0}=u_{0}(x)
\end{array}\right.
$$

in the unbounded domain $\Omega \subset \mathbb{R}^{n}$ satisfying the assumptions of the previous Section. Moreover, based on these estimates, we derive the existence of a solution $u(t)$ for (2.1), its uniqueness and obtain several estimates for differences of solutions of (2.1) which will be used below for studying the attractor of this system.

We recall, that $u(t)=\left(u^{1}(t, x), \cdots, u^{k}(t, x)\right)$ is the vector-valued function, $a$ is the constant $k \times k$-matrix satisfying the condition $a+a^{*}>0, \lambda_{0}>0$, the vector field $L \in C_{b}^{1}\left(\mathbb{R}^{n}, \mathbb{R}^{n}\right)$ satisfies the inequality

$$
\|\operatorname{div} L\|_{0, \infty} \leq \lambda_{0} / 2
$$

and the nonlinear term $f(u)$ satisfies the assumptions

$$
\begin{cases}\text { 1. } & f \in C^{3}\left(\mathbb{R}^{k}, \mathbb{R}^{k}\right) \\ \text { 2. } & f(u) . u \geq-C \\ \text { 3. } & f^{\prime}(u) \geq-K\end{cases}
$$


Moreover, we impose the additional growth restrictions for the nonlinearity $f(u)$ :

$$
\begin{cases}\text { 1. } & |f(u)| \leq C\left(1+|u|^{p}\right) \\ \text { 2. }\left|f^{\prime}(u)\right|^{p /(p-1)} \leq C(1+|f(u)|+|u|),\end{cases}
$$

where the exponent $p>1$ is arbitrary, for $n \leq 4$, and $p<1+\frac{4}{n-4}$, for $n \geq 5$.

We also assume that the external force $g$ belongs to the space $L_{b}^{q}(\Omega)$, for some $q>n+1$, the initial data $u_{0}$ belongs to the space $W_{b}^{2, q}(\Omega)$ and the boundary data $u^{0}$ belongs to the following trace space:

$$
\begin{aligned}
\Psi_{b}\left(\mathbb{R}_{+} \times \partial \Omega\right):=\left.\operatorname{Tr}\right|_{\partial \Omega}\left\{u, \partial_{t} u \in W_{b}^{(1,2), q}\left(\mathbb{R}_{+} \times \Omega\right)\right\} \equiv \\
\equiv\left\{u^{0}, \partial_{t} u^{0} \in W_{b}^{(1-1 /(2 q), 2-1 / q), q}\left(\mathbb{R}_{+} \times \partial \Omega\right)\right\}
\end{aligned}
$$

(see Definition 1.5 and [28]). Moreover, we assume that the first compatibility condition

$$
\left.u_{0}\right|_{\partial \Omega}=\left.u^{0}\right|_{t=0}
$$

is satisfied.

By definition, a solution of (2.1) is a function

$$
u \in L^{\infty}\left(\mathbb{R}_{+}, W_{b}^{2, q}(\Omega)\right) \cap C\left([0, \infty), L_{b}^{q}(\Omega)\right)
$$

which satisfies equation (2.1) in the sense of distributions.

Remark 2.1. It follows from the Sobolev's embedding theorem and from our choice of the exponent $q(q>n / 2)$ that the solution $u$ belongs to $L^{\infty}\left(\mathbb{R}_{+} \times \Omega\right)$, consequently, the nonlinear term in $(2.1)$ is well-defined and belongs to $L^{\infty}$. Therefore, it follows from (2.7) and from equation (2.1) that

$$
\partial_{t} u \in L^{\infty}\left(\mathbb{R}_{+}, L_{b}^{q}(\Omega)\right) .
$$

Moreover, it can be shown by standard arguments (see e.g. [39]) that

$$
u \in C\left([0, T], W_{e^{-\varepsilon|x|}}^{2, q}(\Omega)\right) \cap C^{1}\left([0, T], L_{e^{-\varepsilon|x|}}^{q}(\Omega)\right),
$$

for every $T>0$ and every $\varepsilon>0$. We, however, note that, in contrast to the case of bounded domains, for generic $u_{0} \in \Phi$, the corresponding solution $u(t)$ is not continuous at $t=0$ as a function with values in $W_{b}^{2, q}(\Omega)$ (see e.g. [33] for the conditions on $u_{0}$ which guarantee this continuity).

The main result of this Section is the following theorem.

Theorem 2.1. Let the above assumptions hold and let $u(t)$ be a solution of (2.1). Then, the following estimate is valid:

$$
\begin{aligned}
\left\|u(t), \Omega \cap B_{x_{0}}^{1}\right\|_{2, q} & \leq Q\left(\|u(0)\|_{W_{b}^{2, q}(\Omega)}\right) e^{-\alpha t}+ \\
& +Q\left(\left\|\left.u\right|_{\partial \Omega}\right\|_{\Psi_{b}\left(\mathbb{R}_{+} \times \partial \Omega\right)}\right) e^{-\alpha \operatorname{dist}\left(x_{0}, \partial \Omega\right)}+Q\left(\|g\|_{L_{b}^{q}(\Omega)}\right),
\end{aligned}
$$

where $\alpha$ is a positive constant which depends only on the equation and $Q$ is a monotonic function which also depends only on the equation (and is independent of $u, u_{0}$ and $\left.u^{0}\right)$.

Proof. Estimate (2.9) has been proved in [43], for the particular case of $u^{0}=0$ and $L=0$. In general case, although the presence of nonzero drift term $\left(L, \nabla_{x}\right) u$ is not essential for that proof (due to assumption (2.2)), but the nonhomogeneous boundary conditions require the additional accuracy and the additional technicalities which will be indicated below. 
Lemma 2.1. Let the above assumptions hold. Then, the following estimate is valid, for every $x_{0} \in \Omega, \mu>0$ and for $\varepsilon>0$ small enough:

$$
\begin{aligned}
& \left(|u(T)|^{2}, \phi_{\varepsilon, x_{0}}\right)+\int_{T}^{T+1}\left(\left|\nabla_{x} u(t)\right|^{2}, \phi_{\varepsilon, x_{0}}\right) d t \leq \\
& \leq C e^{-\alpha T}\left(|u(0)|^{2}, \phi_{\varepsilon, x_{0}}\right)+C\left(|g|^{2}, \phi_{\varepsilon, x_{0}}\right)+C+ \\
& \quad+\mu \int_{0}^{T} e^{-\alpha(T-t)}\left(\phi_{\varepsilon, x_{0}},\left|\nabla_{x} u(t)\right|^{2}\right)_{\partial \Omega} d t+C_{\mu} e^{-\varepsilon \operatorname{dist}\left(x_{0}, \partial \Omega\right)}\left\|u^{0}\right\|_{\Psi_{b}}^{2},
\end{aligned}
$$

where the positive constants $C, \alpha, \varepsilon$ are independent of $x_{0}, C_{\mu}$ depends only on $\mu>0$, and $(u, v)$ and $(u, v)_{\partial \Omega}$ stand for the inner product in $L^{2}(\Omega)$ and in $L^{2}(\partial \Omega)$ respectively.

Proof. Multiplying equation (2.1) by $u(t) e^{-\varepsilon\left|x-x_{0}\right|}$ (with $\varepsilon>0$ small enough), integrating by parts and using the dissipativity assumption $f(u) . u \geq-C$, the positiveness of $a$, assumption (2.2) and the obvious estimate

$$
\left\|\nabla_{x}\left(e^{-\varepsilon\left|x-x_{0}\right|}\right)\right\| \leq \varepsilon e^{-\varepsilon\left|x-x_{0}\right|},
$$

we derive that

$$
\begin{aligned}
& \partial_{t}\left(|u(t)|^{2}, \phi_{\varepsilon, x_{0}}\right)+\alpha\left(|u(t)|^{2}, \phi_{\varepsilon, x_{0}}\right)+\alpha\left(\left|\nabla_{x} u(t)\right|^{2}, \phi_{\varepsilon, x_{0}}\right) \leq \\
& \leq C\left(1+\left(|g|^{2}, \phi_{\varepsilon, x_{0}}\right)+\left(\left|u^{0}(t)\right|^{2}, \phi_{\varepsilon, x_{0}}\right)_{\partial \Omega}+\left(\left|u^{0}(t)\right| \cdot\left|\nabla_{n} u(t)\right|, \phi_{\varepsilon, x_{0}}\right)_{\partial \Omega}\right),
\end{aligned}
$$

where $\alpha>0$ is an appropriate positive constant which is independent of $x_{0}$ (see e.g. [17] or [43] for details). Applying the Hölder inequality to the last term in the right-hand side of (2.12) and using inequality (1.16), together with the Gronwall inequality, we obtain the assertion of the lemma.

Lemma 2.2. Let the above assumptions hold. Then, the following estimate is valid, for sufficiently small $\varepsilon>0$ :

$$
\begin{aligned}
& (2.13)\left(|u(T)|^{2}+\left|\nabla_{x} u(T)\right|^{2}, \phi_{\varepsilon, x_{0}}\right)+\int_{T}^{T+1}\left(\left|\Delta_{x} u(t)\right|^{2}, \phi_{\varepsilon, x_{0}}\right) d t \leq C+ \\
& +C e^{-\alpha T}\left(|u(0)|^{2}+\left|\nabla_{x} u(0)\right|^{2}, \phi_{\varepsilon, x_{0}}\right)+C\left(|g|^{2}, \phi_{\varepsilon, x_{0}}\right)+C e^{-\alpha \operatorname{dist}\left(x_{0}, \partial \Omega\right)} Q\left(\left\|u^{0}\right\|_{\Psi_{b}}\right),
\end{aligned}
$$

where the positive constants $C, \alpha$ and the monotonic function $Q$ are independent of $x_{0}$.

Proof. Multiplying equation (2.1) by the expression

$$
\sum_{i=1}^{n} \partial_{x_{i}}\left(\phi_{\varepsilon, x_{0}}(x) \partial_{x_{i}} u(t)\right):=\phi_{\varepsilon, x_{0}} \Delta_{x} u(t)+\nabla_{x} \phi_{\varepsilon, x_{0}} \cdot \nabla_{x} u(t)
$$

and setting $\varepsilon>0$ small enough, we obtain, after the standard integration by parts and using the monotonicity assumption $f^{\prime}(u) \geq-K$ and the inequality (2.11), that

$$
\begin{aligned}
& \partial_{t}\left(\left|\nabla_{x} u(t)\right|^{2}, \phi_{\varepsilon, x_{0}}\right)+\alpha\left(\left|\nabla_{x} u(t)\right|^{2}, \phi_{\varepsilon, x_{0}}\right)+\alpha\left(\left|\Delta_{x} u(t)\right|^{2}, \phi_{\varepsilon, x_{0}}\right) \leq \\
& \leq\left(2 K+C\|L\|_{0, \infty}^{2}\right)\left(\left|\nabla_{x} u(t)\right|^{2}, \phi_{\varepsilon, x_{0}}\right)+C\left(|g|^{2}, \phi_{\varepsilon, x_{0}}\right)+ \\
&+C\left(\left(\left|\partial_{t} u^{0}(t)\right|+\left|f\left(u^{0}(t)\right)\right|\right) \cdot\left|\nabla_{n} u(t)\right|, \phi_{\varepsilon, x_{0}}\right)_{\partial \Omega}
\end{aligned}
$$


We now recall that, due to our choice of the exponent $q, u^{0} \in C_{b}\left(\mathbb{R}_{+} \times \Omega\right)$ and, consequently, the last term in the right-hand side of $(2.15)$ can be estimated as follows:

$$
\begin{aligned}
C\left(\left(\left|\partial_{t} u^{0}(t)\right|+\right.\right. & \left.\left.\left|f\left(u^{0}(t)\right)\right|\right) \cdot\left|\nabla_{n} u(t)\right|, \phi_{\varepsilon, x_{0}}\right)_{\partial \Omega} \leq \\
& \leq C_{\mu} e^{-\varepsilon / 2 \operatorname{dist}\left(x_{0}, \partial \Omega\right)} Q\left(\left\|u^{0}\right\|_{\Psi_{b}}\right)+\mu\left(\left|\nabla_{n} u(t)\right|^{2}, \phi_{\varepsilon, x_{0}}\right)_{\partial \Omega}
\end{aligned}
$$

where the constant $\mu>0$ can be chosen arbitrarily small.

Applying the Gronwall inequality to relation (2.15) and estimating the last two terms in the right-hand side of it by (2.10) and (2.16), we have, after simple computations

$$
\begin{gathered}
\left(|u(T)|^{2}+\left|\nabla_{x} u(T)\right|^{2}, \phi_{\varepsilon, x_{0}}\right)+\int_{T}^{T+1}\left(\left|\Delta_{x} u(t)\right|^{2}, \phi_{\varepsilon, x_{0}}\right) d t \leq \\
\leq C\left(e^{-\alpha t}\left(|u(0)|^{2}+\left|\nabla_{x} u(0)\right|^{2}, \phi_{\varepsilon, x_{0}}\right)+1+\left(|g|^{2}, \phi_{\varepsilon, x_{0}}\right)\right)+ \\
+C_{\mu} e^{-\alpha \operatorname{dist}\left(x_{0}, \partial \Omega\right)} Q\left(\left\|u^{0}\right\|_{\Psi_{b}}\right)+\mu \sup _{t \in[0, T]} e^{-\alpha(T-t)} \int_{t}^{t+1}\left(\left|\nabla_{n} u(s)\right|^{2}, \phi_{\varepsilon, x_{0}}\right)_{\partial \Omega} d s
\end{gathered}
$$

where $Q$ is an appropriate monotonic function, $\alpha>0$ is a certain positive constant and $\mu>0$ is arbitrary.

Due to the regularity theorem for the Laplace operator in weighted Sobolev spases (see, e.g. [35]), we have

$$
\|u(t)\|_{W_{\phi_{\varepsilon}, x_{0}}^{2,2}(\Omega)} \leq C\left(\left\|\Delta_{x} u(t)\right\|_{L_{\phi_{\varepsilon}, x_{0}}^{2}(\Omega)}+\|u(t)\|_{L_{\phi_{\varepsilon, x_{0}}}^{2}(\Omega)}+\|u(t)\|_{W_{\phi_{\varepsilon, x_{0}}}^{3 / 2,2}(\partial \Omega)}\right)
$$

and, consequently, the last term in (2.17) can be estimated as follows:

$$
\begin{aligned}
\left(\left|\nabla_{n} u(s)\right|^{2}, \phi_{\varepsilon, x_{0}}\right)_{\partial \Omega} \leq C\left(\left|\Delta_{x} u(s)\right|^{2}, \phi_{\varepsilon, x_{0}}\right)+ & C\left(|u(s)|^{2}, \phi_{\varepsilon, x_{0}}\right)+ \\
& +C e^{-\varepsilon / 2 \operatorname{dist}\left(x_{0}, \partial \Omega\right)}\left\|u^{0}\right\|_{\Psi_{b}}^{2} .
\end{aligned}
$$

Inserting this estimate to the right-hant of (2.17) and using (2.10), we derive

$$
\begin{gathered}
\left(|u(T)|^{2}+\left|\nabla_{x} u(T)\right|^{2}, \phi_{\varepsilon, x_{0}}\right)+\int_{T}^{T+1}\left(\left|\Delta_{x} u(t)\right|^{2}, \phi_{\varepsilon, x_{0}}\right) d t \leq \\
\leq C_{1}\left(e^{-\alpha t}\left(|u(0)|^{2}+\left|\nabla_{x} u(0)\right|^{2}, \phi_{\varepsilon, x_{0}}\right)+1+\left(|g|^{2}, \phi_{\varepsilon, x_{0}}\right)\right)+ \\
+C_{\mu}^{\prime} e^{-\alpha \operatorname{dist}\left(x_{0}, \partial \Omega\right)} Q\left(\left\|u^{0}\right\|_{\Psi_{b}}\right)+C \mu \sup _{t \in[0, T]} e^{-\alpha(T-t)} \int_{t}^{t+1}\left(\left|\Delta_{x} u(s)\right|^{2}, \phi_{\varepsilon, x_{0}}\right) d s .
\end{gathered}
$$

There remains to note that estimate (2.20) implies (2.13) in a standard way, if $C \mu<1 / 2$ (see [17] or [42]) and Lemma 2.2 is proved.

Our next task is to obtain the analogous to (2.13) estimate for the $W_{\phi_{\varepsilon, x_{0}}}^{2,2}$-norm. To this end, we introduce the following 'norm', which depends on $\varepsilon>0$ and $x_{0} \in \Omega$ :

$$
\|v\|_{D_{\varepsilon, x_{0}}}^{2}:=\|v\|_{W_{\phi_{\varepsilon}, x_{0}}^{2,2}(\Omega)}^{2}+\|f(v)\|_{L_{\phi_{\varepsilon, x_{0}}}^{2}(\Omega)}^{2} .
$$


Lemma 2.3. Let the above assumptions hold and let $\varepsilon>0$ be small enough. Then, the following estimate is valid for the solutions of equation (2.1):

$$
\begin{aligned}
& \|u(t)\|_{D_{\varepsilon, x_{0}}}^{2} \leq \\
\leq & C e^{(2 K+1) t}\left(\|u(0)\|_{D_{\varepsilon, x_{0}}}^{2}+1+\|g\|_{L_{\phi_{\varepsilon, x_{0}}}^{2}(\Omega)}^{2}+e^{-\alpha \operatorname{dist}\left(x_{0}, \partial \Omega\right)} Q\left(\left\|u^{0}\right\|_{\Psi_{b}}\right)\right),
\end{aligned}
$$

where the constant $K$ is the same as in (2.3), $\alpha>0$ and the constant $C$ and the function $Q$ are independent of $x_{0}$ and $\varepsilon$.

Proof. We give below only the formal deducing of estimate (2.22) which can be justified in a the standard way (using, e.g. the uniqueness of a solution of (2.1) in the class (2.7) which is verified in Theorem 2.2).

We differentiate equation (2.1) with respect to $t$ and denote $\theta(t):=\partial_{t} u(t)$. Then, this function satisfies the following equation:

$$
\left\{\begin{array}{l}
\partial_{t} \theta=a \Delta_{x} \theta-\left(L, \nabla_{x}\right) \theta-\lambda_{0} \theta-f^{\prime}(u) \theta \\
\theta(0)=a \Delta_{x} u_{0}-\left(L, \nabla_{x}\right) u_{0}-\lambda_{0} u_{0}-f\left(u_{0}\right)+g,\left.\quad \theta\right|_{\partial \Omega}=\partial_{t} u^{0}
\end{array}\right.
$$

Let us now fix the extention $w(t)\left(w(t), \partial_{t} w(t) \in W_{b}^{(1,2), q}\left(\mathbb{R}_{+} \times \Omega\right)\right)$ of the boundary condition $u^{0} \in \Psi_{b}$ inside of the domain $\Omega$ such that

$$
\begin{cases}\text { 1. } & \|w\|_{W_{b}^{(1,2), q}\left(\mathbb{R}^{+} \times \Omega\right)}+\left\|\partial_{t} w\right\|_{W_{b}^{(1,2), q}\left(\mathbb{R}^{+} \times \Omega\right)} \leq C\left\|u^{0}\right\|_{\Psi_{b}} \\ \text { 2. } & w(t, x) \equiv 0, \text { if } \operatorname{dist}\left(x_{0}, \Omega\right) \geq 1\end{cases}
$$

The existence of such an extention is and immediate corollary of the definition of $\Psi_{b}$ (see (2.5)). Then, the function $\theta_{1}(t):=\theta(t)-\partial_{t} w(t)$ satisfies the following equation:

$$
\left\{\begin{array}{l}
\partial_{t} \theta_{1}=a \Delta_{x} \theta_{1}-\left(L, \nabla_{x}\right) \theta_{1}-f^{\prime}(u) \theta_{1}+h_{w}(t)-f^{\prime}(u) \partial_{t} w \\
\left.\theta_{1}\right|_{t=0}=\left.\theta\right|_{t=0}-\left.\partial_{t} w\right|_{t=0},\left.\quad \theta_{1}\right|_{\partial \Omega}=0
\end{array}\right.
$$

where $h_{w}(t):=\partial_{t}^{2} w(t)-a \Delta_{x} \partial_{t} w(t)-\left(L, \nabla_{x}\right) \partial_{t} w(t)-\lambda_{0} \partial_{t} w(t)$. Multiplying (2.25) by $\theta_{1}(t) \phi_{\varepsilon, x_{0}}$, integrating over $\Omega$ and using that $\varepsilon>0$ is small enough and assumption $f^{\prime}(u) \geq K$, we derive, after the standard estimates, that

$$
\begin{aligned}
& \frac{1}{2}\left(\left|\theta_{1}(t)\right|^{2}, \phi_{\varepsilon, x_{0}}\right)+\frac{\lambda_{0}}{4}\left(\left|\theta_{1}(t)\right|^{2}, \phi_{\varepsilon, x_{0}}\right) \leq \\
& \leq K\left(\left|\theta_{1}(t)\right|^{2}, \phi_{\varepsilon, x_{0}}\right)+C\left(\left|h_{w}(t)\right|^{2}, \phi_{\varepsilon, x_{0}}\right)-\left(f^{\prime}(u(t)) \partial_{t} w(t) . \theta_{1}(t), \phi_{\varepsilon, x_{0}}\right),
\end{aligned}
$$

where the constant $C>0$ is independent of $x_{0}$. Estimating the last term in (2.26) by Hölder inequality and using assumption $(2.4)(2)$, the embedding $W_{b}^{(1,2), q}\left(\mathbb{R}_{+} \times \Omega\right) \subset$ $C\left(\mathbb{R}_{+} \times \Omega\right)$ and estimates (2.24), we have

$$
\begin{aligned}
\left(f^{\prime}(u) \partial_{t} w \cdot \theta_{1}, \phi_{\varepsilon, x_{0}}\right) \leq \mu & \left(\left|f^{\prime}(u)\right|^{2 p /(p-1)}, \phi_{\varepsilon, x_{0}}\right)+\lambda_{0} / 4\left(\left|\theta_{1}\right|^{2}, \phi_{\varepsilon, x_{0}}\right)+ \\
+C_{\mu}\left(\left|\partial_{t} w\right|^{2 p}, \phi_{\varepsilon, x_{0}}\right) \leq & \mu\left(|f(u)|^{2}, \phi_{\varepsilon, x_{0}}\right)+C\left(1+\left(|u|^{2}, \phi_{\varepsilon, x_{0}}\right)\right)+ \\
& +\lambda_{0} / 4\left(\left|\theta_{1}\right|^{2}, \phi_{\varepsilon, x_{0}}\right)+C_{\mu} e^{-\alpha \operatorname{dist}\left(x_{0}, \partial \Omega\right)}\left\|u^{0}\right\|_{\Psi_{b}}^{2 p}
\end{aligned}
$$


where the constant $\mu>0$ can be chosen arbitrarily small.

Inserting estimate (2.27) to the right-hand side of (2.26), applying the Gronwall inequality to the obtained relation and using (2.13) and (2.24) (in order to estimate the function $\left.\partial_{t} h_{w}(t)\right)$, we derive that

$$
\begin{aligned}
& \left(\left|\partial_{t} u(T)\right|^{2}, \phi_{\varepsilon, x_{0}}\right) \leq C e^{2 K T}\left(\|u(0)\|_{D_{\varepsilon, x_{0}}}^{2}+1+\left(|g|^{2}, \phi_{\varepsilon, x_{0}}\right)\right)+ \\
& +C_{\mu} e^{2 K T-\alpha \operatorname{dist}\left(x_{0}, \partial \Omega\right)} Q\left(\left\|u^{0}\right\|_{\Psi_{b}}\right)+\mu \int_{0}^{T} e^{2 K(T-t)}\left(|f(u(t))|^{2}, \phi_{\varepsilon, x_{0}}\right) d t,
\end{aligned}
$$

where the constant $\mu$ can be chosen arbitrarily small.

After obtaining estimate (2.28) for the $L^{2}$-norm of the $t$-derivative, we may consider parabolic equation (2.1) as an elliptic boundary value problem at a fixed point $T$ :

$$
a \Delta_{x} u(T)-\left(L, \nabla_{x}\right) u(T)-f(u(T))=h_{u}:=\partial_{t} u(T)-g,\left.\quad u(T)\right|_{\partial \Omega}=u^{0}(T),
$$

with the right-hand side $h_{u}$ belonging to the space $L_{\phi_{\varepsilon, x_{0}}}^{2}(\Omega)$. Arguing as in the proof of Lemma 2.2 (multiplying the equation by expression (2.14) and using estimate (2.18)), we derive the following estimate:

$$
\|u(T)\|_{W_{\phi_{\varepsilon}, x_{0}}^{2,2}(\Omega)}^{2} \leq C\left(1+\left\|h_{u}\right\|_{L_{\phi_{\varepsilon}, x_{0}}^{2}(\Omega)}^{2}+e^{-\alpha \operatorname{dist}\left(x_{0}, \partial \Omega\right)} Q\left(\left\|u^{0}\right\|_{\Psi_{b}}\right)\right) .
$$

Estimates (2.28) and (2.30) and equation (2.1) imply now that

$$
\begin{gathered}
\left(|f(u(T))|^{2}, \phi_{\varepsilon, x_{0}}\right) \leq C_{1} e^{2 K T}\left(\|u(0)\|_{D_{\varepsilon, x_{0}}}^{2}+1+\left(|g|^{2}, \phi_{\varepsilon, x_{0}}\right)\right)+ \\
+C_{\mu}^{\prime} e^{2 K T-\alpha \operatorname{dist}\left(x_{0}, \partial \Omega\right)} Q\left(\left\|u^{0}\right\|_{\Psi_{b}}\right)+C_{2} \mu \int_{0}^{T} e^{2 K(T-t)}\left(|f(u(t))|^{2}, \phi_{\varepsilon, x_{0}}\right) d t .
\end{gathered}
$$

Setting $\mu=1 / C_{2}$ and applying the Gronwall inequality to relation (2.31), we finally have

$$
\begin{aligned}
\left(|f(u(T))|^{2}, \phi_{\varepsilon, x_{0}}\right) \leq C_{3} e^{(2 K+1) T}\left(\|u(0)\|_{D_{\varepsilon, x_{0}}}^{2}+1+\left(|g|^{2}, \phi_{\varepsilon, x_{0}}\right)\right)+ \\
+C_{4} e^{(2 K+1) T} e^{-\alpha \operatorname{dist}\left(x_{0}, \partial \Omega\right)} Q\left(\left\|u^{0}\right\|_{\Psi_{b}}\right) .
\end{aligned}
$$

Combining this estimate with (2.28) and (2.30), we obtain estimate (2.22) which proves Lemma 2.3.

We now note that the obtained estimate of the $W_{\phi_{\varepsilon, x_{0}}}^{2,2}$-norm diverges exponentially with respect to $t \rightarrow \infty$ which is not good from the attractors point of view. In order to remove this divergence, we need the following smoothing property.

Lemma 2.4. Let the above assumptions hold. Then, the following estimate is valid, for any solution $u(t)$ of problem (2.1):

$$
\begin{aligned}
& \|u(1)\|_{W_{\phi p \varepsilon, x_{0}}^{2,2}(\Omega)}^{2} \leq \\
& \quad \leq C\left(1+\|u(0)\|_{W_{\phi_{\varepsilon}, x_{0}}^{1,2}(\Omega)}^{2 p}+\|g\|_{L_{\phi_{\varepsilon}, x_{0}}^{2}(\Omega)}^{2 p}+e^{-\alpha \operatorname{dist}\left(x_{0}, \partial \Omega\right)} Q\left(\left\|u^{0}\right\|_{\Psi_{b}}\right)\right),
\end{aligned}
$$


for a certain monotonic function $Q$, positive constant $\alpha>0$ and for a sufficiently small positive $\varepsilon>0$.

Proof. Let us fix an arbitrary $x_{0} \in \Omega$ and a sufficiently small $\varepsilon>0$. Then, it follows from estimates (2.13) and (2.18) that

$$
\begin{aligned}
& \int_{0}^{1}\|u(t)\|_{W_{\phi_{\varepsilon}, x_{0}}^{2,2}(\Omega)}^{2} d t \leq \\
& \quad \leq C\left(1+\|u(0)\|_{W_{\phi_{\varepsilon}, x_{0}}^{1,2}(\Omega)}^{2}+\|g\|_{L_{\phi_{\varepsilon}, x_{0}}^{2}(\Omega)}^{2}+e^{-\alpha \operatorname{dist}\left(x_{0}, \partial \Omega\right)} Q\left(\left\|u^{0}\right\|_{\Psi_{b}}\right)\right)
\end{aligned}
$$

It follows from (2.33) that there exists a point $T=T\left(x_{0}\right) \in[0,1]$ such that

$$
\begin{aligned}
& \|u(T)\|_{W_{\phi_{\varepsilon}, x_{0}}^{2,2}(\Omega)}^{2} \leq \\
& \quad \leq C\left(1+\|u(0)\|_{W_{\phi_{\varepsilon}, x_{0}}^{1,2}(\Omega)}^{2}+\|g\|_{L_{\phi_{\varepsilon}, x_{0}}^{2}(\Omega)}^{2}+e^{-\alpha \operatorname{dist}\left(x_{0}, \partial \Omega\right)} Q\left(\left\|u^{0}\right\|_{\Psi_{b}}\right)\right) .
\end{aligned}
$$

Moroeover, it is proved in [43] that the first growth restriction of (2.4), together with Sobolev embedding theorem, imply the following estimate:

$$
\|f(u(T))\|_{L_{\phi_{p \varepsilon, x_{0}}}^{2}(\Omega)}^{2} \leq C\left(1+\|u(T)\|_{W_{\phi_{\varepsilon, x_{0}}}^{2,2}(\Omega)}^{2 p}\right)
$$

Estimates (2.34) and (2.35) imply that

$$
\begin{aligned}
& \|u(T)\|_{D_{p \varepsilon, x_{0}}}^{2} \leq \\
& \quad \leq C_{1}\left(1+\|u(0)\|_{W_{\phi_{\varepsilon}, x_{0}}^{1,2}(\Omega)}^{2 p}+\|g\|_{L_{\phi_{\varepsilon, x_{0}}}^{2}(\Omega)}^{2 p}+e^{-\alpha \operatorname{dist}\left(x_{0}, \partial \Omega\right)} Q\left(\left\|u^{0}\right\|_{\Psi_{b}}\right)\right) .
\end{aligned}
$$

Applying now estimate (2.22) (where $\varepsilon$ is replaced by $p \varepsilon$ ) at the initial moment $t=T$ instead of $t=0$ and using (2.36), we derive estimate (2.32). Lemma 2.4 is proved.

Thus, we have proved the analogue of estimate (2.9) for $q=2$.

Lemma 2.5. Let the above assumptions hold. Then

$$
\begin{aligned}
\| u(t), \Omega & \cap B_{x_{0}}^{1} \|_{2,2} \leq \\
& \leq Q\left(\left\|u_{0}\right\|_{W_{b}^{2,2}(\Omega)}\right) e^{-\alpha t}+Q\left(\|g\|_{L_{b}^{2}(\Omega)}\right)+e^{-\alpha \operatorname{dist}\left(x_{0}, \Omega\right)} Q\left(\left\|u^{0}\right\|_{\Psi_{b}}\right),
\end{aligned}
$$

for some positive $\alpha$ and certain monotonic function $Q$.

Indeed, this lemma is a simple corollary of estimates (2.13), (2.22) and (2.32).

Estimate $(2.9)$ of the $W^{2, q}$-norm of the solution $u$ can be now deduced from (2.37) in a standard way, using the regularity theorems for the linear parabolic equation and the first growth condition of (2.4) for improving steps by steps the regularity of solution $u(t)$ (see [43]). Theorem 2.1 is proved. 
Corollary 2.1. Let the assumptions of Theorem 2.1 hold. Then, the following estimate is valid:

$$
\|u(t)\|_{W_{b}^{2, q}(\Omega)} \leq Q\left(\|u(0)\|_{W_{b}^{2, q}(\Omega)}\right) e^{-\alpha t}+Q\left(\left\|u^{0}\right\|_{\Psi_{b}}\right)+Q\left(\|g\|_{L_{b}^{q}(\Omega)}\right)
$$

for the appropriate monotonic function $Q$ and positive constant $\alpha>0$.

Indeed, estimate (2.38) is an immediate corollary of estimate (2.9).

As usual, after obtaining a priori estimate (2.9), one can easily verify the existence of a solution for problem (2.1).

Theorem 2.2. Let the above assumptions hold. Then, for every $u_{0} \in W_{b}^{2, q}(\Omega)$ which satisfies the compatibility condition (2.6), equation (2.1) possesses a unique solution $u(t)$. Moreover, the following estimate holds, for every two solutions $u_{1}(t)$ and $u_{2}(t)$ of equation (2.1):

$$
\begin{aligned}
\left\|u_{1}(T)-u_{2}(T)\right\|_{L_{\phi_{\varepsilon}, x_{0}}^{2}(\Omega)}^{2}+\int_{T}^{T+1}\left\|u_{1}(t)-u_{2}(t)\right\|_{W_{\phi_{\varepsilon}, x_{0}}^{1,2}(\Omega)}^{2} d t \leq \\
\leq C e^{2 K T}\left\|u_{1}(0)-u_{2}(0)\right\|_{L_{\phi_{\varepsilon, x_{0}}}^{2}(\Omega)}^{2}
\end{aligned}
$$

where the constant $K$ is the same as in (2.3), $\varepsilon>0$ is a small parameter, and the constant $C$ depends only on the equation.

Proof. The existence of a solution of (2.1), in case $\Omega$ is bounded, can be deduced from a priori estimate (2.38) using the Leray-Schauder fixed point principle (see e.g. [37]). The existence of a solution in the unbounded domain $\Omega$ can be proved after that, approximating the unbounded domain $\Omega$ by the bounded ones $\Omega_{N}$ and passing to the limit $N \rightarrow \infty$ (see e.g. [17] or [43] for the details).

Let us now prove estimate (2.39) which immediately implies the uniqueness. Let $u_{1}(t)$ and $u_{2}(t)$ be two solutions of $(2.1)$ and let $v(t)=u_{1}(t)-u_{2}(t)$. Then, this function satisfies the equation

$$
\partial_{t} v=a \Delta_{x} v-\left(L, \nabla_{x}\right) v-\lambda_{0} v-l(t) v,\left.\quad v\right|_{\partial \Omega}=0,\left.\quad v\right|_{t=0}=u_{1}(0)-u_{2}(0)
$$

where $l(t):=\int_{0}^{1} f^{\prime}\left(s u_{1}(t)+(1-s) u_{2}(t)\right) d s$. We note that, according to our assumptions on $f$, we have $l(t) \geq-K$, consequently, multiplying equation (2.40) by $v(t) \phi_{\varepsilon, x_{0}}$, integrating over the $x \in \Omega$ and arguing as in the proof of Lemma 2.1, we derive estimate (2.39) and finishes the proof of Theorem 2.2.

Corollary 2.2. Let the above assumptions hold and let the boundary condition $u^{0} \in \Psi_{b}\left(\mathbb{R}_{+} \times \Omega\right)$ be independent of $t$ (i.e. $u^{0}(t, x) \equiv u^{0}(x) \in W_{b}^{2-1 / q, q}(\partial \Omega)$ ). Then, problem (2.1) generates a semigroup $\left\{S_{t}, t \geq 0\right\}$ in the phase space $\Phi_{b}(\Omega):=$ $W_{b}^{2, q}(\Omega) \cap\left\{\left.u_{0}\right|_{\partial \Omega}=u^{0}\right\}:$

$$
S_{t}: \Phi_{b}(\Omega) \rightarrow \Phi_{b}(\Omega), \quad u(t)=S_{t} u_{0},
$$

where $u(t)$ is the solution of (2.1) with $u(0)=u_{0}$. 
Theorem 2.3. Let the assumptions of Theorem 2.1 hold. Then, for every two solutions $u_{1}(t), u_{2}(t) \in \Phi_{b}$ and for every $\varepsilon>0$, the following estimate is valid:

$$
\left\|u_{1}(t)-u_{2}(t)\right\|_{W_{\phi_{\varepsilon}, x_{0}}^{2, q}(\Omega)} \leq C e^{K t}\left\|u_{1}(0)-u_{2}(0)\right\|_{W_{\phi_{\varepsilon}, x_{0}}^{2, q}(\Omega)}
$$

where the constants $C, K$ depend on $\left\|u_{i}\right\|_{W_{b}^{2, q}},\left\|u^{0}\right\|_{\Psi_{b}}$ and $\varepsilon$, but are independent of $x_{0} \in \Omega$.

Moreover, the following version of smoothing property is valid for solutions $u_{i}(t)$ :

$$
\left\|u_{1}(1)-u_{2}(1)\right\|_{W_{\phi_{\varepsilon}, x_{0}}^{2, q}(\Omega)} \leq C_{1}\left\|u_{1}(0)-u_{2}(0)\right\|_{L_{\phi_{\varepsilon}, x_{0}}^{q}(\Omega)}
$$

where $C_{1}$ is also independent of $x_{0} \in \Omega$.

Proof. The proof of these estimates is based on a standard analysis of linear equation (2.40) and can be obtained in the spirit of the proof of Theorem 2.1, but essentially simpler, since equation (2.40) is linear and the coefficient $l(t)$ is smooth enough:

$$
\|l(t)\|_{W_{b}^{1, q} \cap C_{b}(\Omega)} \leq Q\left(\left\|u_{i}(0)\right\|_{W_{b}^{2, q}},\left\|u^{0}\right\|_{\Psi_{b}}\right)
$$

(thanks to (2.9) and due to the facts that $f \in C^{2}$ and $W_{b}^{2, q} \subset C$, see e.g. [17] or [43] for the details).

\section{$\S 3$ The global attractor And UpPeR BOUNDS OF ITS KOLMOGOROV'S $\varepsilon$-ENTROPY.}

In this Section, we will only consider the autonomous case of equation (2.1), i.e. we assume that

$$
u^{0}(t, x) \equiv u^{0}(x) \in W_{b}^{2-1 / q, q}(\partial \Omega)
$$

Then, according to Corollary 2.2, this equation generates a semigroup $\left\{S_{t}, t \geq 0\right\}$ in the phase space $\Phi_{b}(\Omega)$ by expression (2.41). Moreover, according to estimate (2.38), this semigroup possesses a bounded absorbing set $\mathcal{B}$ in the phase space $\Phi_{b}(\Omega)$, i.e., for any other bounded subset $B \subset \Phi_{b}(\Omega)$, there exists $T=T(B)$ such that

$$
S_{t} B \subset \mathcal{B} \text { if } t \geq T
$$

but, nevertheless, in contrast of the case of bounded domains, the compact attractor in $\Phi_{b}(\Omega)$ for equation (2.1) may not exist in the case of unbounded domains, e.g. the Chafee-Infante equation in $\mathbb{R}^{n}\left(k=1, f(u)=u^{3}-\lambda u, \lambda>\lambda_{0}\right)$ does not possess a compact attractor in the topology of $W_{b}^{2, q}\left(\mathbb{R}^{n}\right)$ (see, e.g. [39]).

That is the reason why (following [20], [21], [31], [32], [33]), we consider below the attractor $\mathcal{A}$ of semigroup (2.41) which attracts bounded subsets of $\Phi_{b}(\Omega)$ only in a local topology of the space $\Phi_{l o c}=W_{l o c}^{2, q}(\Omega)$ (i.e., $\mathcal{A}$ is the $\left(\Phi_{b}, \Phi_{l o c}\right.$ )-attractor of (2.41) in notations of [4]).

We recall that the space $\Phi_{l o c}(\Omega)$ is reflexive metrizable F-space which is generated by semi-norms $\left\|\cdot \Omega \cap B_{x_{0}}^{1}\right\|_{2, q}, x_{0} \in \Omega$. 
Definition 3.1. A set $\mathcal{A} \subset \Phi_{b}(\Omega)$ is the attractor of the semigroup $S_{t}$ if the following assumptions hold:

1. The set $\mathcal{A}$ is compact in $\Phi_{\text {loc }}(\Omega)$.

2. The set $\mathcal{A}$ is strictly invariant with respect to $S_{t}$, i.e.

$$
S_{t} \mathcal{A}=\mathcal{A}, \text { for } t \geq 0 \text {. }
$$

3. The set $\mathcal{A}$ is an attracting set for $S_{t}$ in local topology, i.e., for every neighborhood $\mathcal{O}(\mathcal{A})$ of $\mathcal{A}$ in the topology of the space $\Phi_{\text {loc }}(\Omega)$ and for every bounded in uniform topology subset $B \subset \Phi_{b}(\Omega)$, there exists $T=T(\mathcal{O}, B)$ such that

$$
S_{t} B \subset \mathcal{O}(\mathcal{A}) \text { if } t \geq T \text {. }
$$

We also recall that the first condition means that the restriction $\left.\mathcal{A}\right|_{\Omega_{1}}$ is compact in the space $W^{2, q}\left(\Omega_{1}\right)$, for every bounded $\Omega_{1} \subset \Omega$.

Analogously, the third condition means that, for every bounded $\Omega_{1} \subset \Omega$, every bounded $B$ in $\Phi_{b}(\Omega)$ and every $W^{2, q}\left(\Omega_{1}\right)$-neighborhood $\mathcal{O}\left(\left.\mathcal{A}\right|_{\Omega_{1}}\right)$ of the restriction $\left.\mathcal{A}\right|_{\Omega_{1}}$, there exists $T=T\left(\Omega_{1}, \mathcal{O}, B\right)$ such that

$$
\left.\left(S_{t} B\right)\right|_{\Omega_{1}} \subset \mathcal{O}\left(\left.\mathcal{A}\right|_{\Omega_{1}}\right) \text { if } t \geq T \text {. }
$$

Theorem 3.1. Let the assumptions of Theorem 2.1 hold and let, in addition, (3.1) be satisfied. Then, the semigroup $S_{t}$, defined by (2.41), possesses an attractor $\mathcal{A}$, in the sense of Definition 3.1, which has the following structure:

$$
\mathcal{A}=\left.\mathcal{K}\right|_{t=0},
$$

where we denote by $\mathcal{K}$ the set of all solutions $u$ of (2.1), defined and bounded for all $t \in \mathbb{R}\left(\sup _{t \in \mathbb{R}}\|u(t)\|_{\Phi_{b}(\Omega)}<\infty\right)$.

The proof of this theorem is more or less standard and given, e.g. in [43] for the particular case $L(x) \equiv 0$ and $u^{0}(x) \equiv 0$. The proof in general case is completely analogous, so we omit it here.

We recall that the attractor $\mathcal{A}$, constructed in Theorem 3.1, is not compact in the phase space $\Phi_{b}(\Omega)$, but only its restrictions $\left.\mathcal{A}\right|_{\Omega \cap B_{x_{0}}^{R}}$ are compact in $W^{2, q}\left(\Omega \cap B_{x_{0}}^{R}\right)$, for every $R>0$ and $x_{0} \in \Omega$. Moreover, in contrast to the case of bounded domains, the fractal dimension of these restrictions may be infinite in many physically relevant examples (it will be the case, e.g. for the Chafee-Infante equation in $\mathbb{R}^{n}$ mentioned above (see e.g. [39])). That is the reason why, following [38], [39] and [41], we study the Kolmogorov's $\varepsilon$-entropy of these restrictions and its dependence on three parameters $\varepsilon, R$ and $x_{0}$.

For the convenience of the reader, we recall below the definition of Kolmogorov's $\varepsilon$-entropy. For the detailed study of this concept, see [27] and [35].

Definition 3.1. Let $\mathbb{M}$ be a metric space and let $K$ be a precompact subset in it. For a given $\varepsilon>0$, let $N_{\varepsilon}(K)=N_{\varepsilon}(K, \mathbb{M})$ be the minimal number of $\varepsilon$-balls in $\mathbb{M}$ which cover the set $K$ (this number is, obviously, finite by Hausdorff criteria). By definition, Kolmogorov's $\varepsilon$-entropy of $K$ in $\mathbb{M}$ is the following number:

$$
\mathbb{H}_{\varepsilon}(K)=\mathbb{H}_{\varepsilon}(K, \mathbb{M}):=\ln N_{\varepsilon}(K)
$$


The fractal dimension $\operatorname{dim}_{F}(K, \mathbb{M})$ can be defined as follows:

$$
\operatorname{dim}_{F}(K)=\operatorname{dim}_{F}(K, \mathbb{M}):=\limsup _{\varepsilon \rightarrow 0} \frac{\mathbb{H}_{\varepsilon}(K)}{\ln \frac{1}{\varepsilon}}
$$

Remark 3.1. We note that the fractal dimension $\operatorname{dim}_{F}(K)$ may be infinite for sufficiently large compacts $K$, but the Kolmogorov's $\varepsilon$-entropy is finite, for every $\varepsilon>0$ (due to the Hausdorff criteria). In particular, it is finite for the restrictions $\left.\mathcal{A}\right|_{\Omega \cap B_{x_{0}}^{R}}$ of the attractor $\mathcal{A}$ constructed in Theorem 3.1.

The following theorem gives the universal upper bounds of the $\varepsilon$-entropy for these restrictions.

Theorem 3.2. Let the assumptions Theorem 3.1 be valid and let

$$
\operatorname{vol}_{\Omega, x_{0}}(R)=\operatorname{vol}\left(\Omega \cap B_{x_{0}}^{R}\right) .
$$

Then, for every $R \in \mathbb{R}_{+}, x_{0} \in \Omega$, and $\varepsilon \leq \varepsilon_{0}<1$

$$
\mathbb{H}_{\varepsilon}\left(\left.\mathcal{A}\right|_{\Omega \cap B_{x_{0}}^{R}}, W_{b}^{2, q}\left(\Omega \cap B_{x_{0}}^{R}\right)\right) \leq C \operatorname{vol}_{\Omega, x_{0}}\left(R+K \ln \frac{1}{\varepsilon}\right) \ln \frac{1}{\varepsilon}
$$

where the constants $C, K$ and $\varepsilon_{0}$ are independent of $R$ and $x_{0} \in \Omega$.

Estimate (3.6) is, in fact, a corollary of the smoothing property (2.43) for differeces of solutions belonging to the attractor $\mathcal{A}$ (see e.g. [39] or [42] for the detailed proof).

Let us formulate now several corollaries of estimate (3.6) (see also [39] or [42]).

Corollary 3.1. Since $C_{b}(\Omega) \subset W_{b}^{2, q}(\Omega)$, then

$$
\mathbb{H}_{\varepsilon}\left(\mathcal{A}, C\left(\Omega \cap B_{x_{0}}^{R}\right)\right) \leq C \operatorname{vol}_{\Omega, x_{0}}\left(R+K \ln \frac{1}{\varepsilon}\right) \ln \frac{1}{\varepsilon}
$$

Corollary 3.2. Let $\Omega=\mathbb{R}^{n}$. Then, $\operatorname{vol}_{\Omega, x_{0}}(r)=c r^{n}$ and, consequently

$$
\mathbb{H}_{\varepsilon}\left(\mathcal{A}, W_{b}^{2, q}\left(B_{x_{0}}^{R}\right)\right) \leq \tilde{C}\left(R+K \ln \frac{1}{\varepsilon}\right)^{n} \ln \frac{1}{\varepsilon}
$$

Setting $R:=M \ln \frac{1}{\varepsilon}, M>0$, we have

$$
\mathbb{H}_{\varepsilon}\left(\mathcal{A}, W_{b}^{2, q}\left(B_{x_{0}}^{M \ln \frac{1}{\varepsilon}}\right)\right) \leq C_{M}\left(\ln \frac{1}{\varepsilon}\right)^{n+1}
$$

We note that estimate (3.8) gives the same type of upper bounds for $R=1$ and $R=M \ln \frac{1}{\varepsilon}$.

Corollary 3.3. Let $\Omega$ be a bounded domain. Then, Theorem 3.1 implies the following estimate:

$$
\mathbb{H}_{\varepsilon}\left(\mathcal{A}, W_{b}^{2, q}(\Omega)\right) \leq C \operatorname{vol}(\Omega) \ln \frac{1}{\varepsilon}
$$


which reflects the well-known fact that, in this case, the attractor $\mathcal{A}$ has the finite fractal dimension.

Corollary 3.4. Let $\Omega=\mathbb{R}^{k} \times \omega^{n-k}$ be a cylindrical domain ( $\omega$ is bounded). Then, estimate (3.6) gives the following bound of the $\varepsilon$-entropy of the attractor $\mathcal{A}$ :

$$
\mathbb{H}_{\varepsilon}\left(\mathcal{A}, W_{b}^{2, q}\left(\Omega \cap B_{x_{0}}^{R}\right)\right) \leq C\left(R+K \ln \frac{1}{\varepsilon}\right)^{k} \ln \frac{1}{\varepsilon}
$$

Corollary 3.5. Let the assumptions of Theorem 3.1 hold, $\Omega=\mathbb{R}^{n}$, and let $M>0$. Then

$$
\mathbb{H}_{\varepsilon}\left(\mathcal{A}, W_{e^{-M|x|}}^{2, q}(\Omega)\right) \leq C(M)\left(\ln \frac{1}{\varepsilon}\right)^{n+1}
$$

Proof. Since the attractor $\mathcal{A}$ is bounded in $W_{b}^{2, q}(\Omega)$, then there exists a number $R=R(M)$ such that

$$
\|\mathcal{A},\{|x|>R \ln 1 / \varepsilon\}\|_{e^{-M|x|}, 2, q} \leq \varepsilon / 2
$$

and, consequently

$$
\mathbb{H}_{\mathcal{E}}\left(\mathcal{A}, W_{e^{-M|x|}}^{2, q}(\Omega)\right) \leq \mathbb{H}_{\mathcal{\varepsilon} / 2}\left(\left.\mathcal{A}\right|_{\left.B_{0}^{R \ln 1 / \varepsilon}, W^{2, q}\left(B_{0}^{R \ln 1 / \varepsilon}\right)\right)}\right.
$$

Thus, there remains to estimate the entropy in the right-hand side of (3.14). To this end, we note that

$$
\left\|v, B_{0}^{R \ln 1 / \varepsilon}\right\|_{2, q} \leq(C R \ln 1 / \varepsilon)^{n / q}\left\|v, B_{0}^{R \ln 1 / \varepsilon}\right\|_{b, 2, q}
$$

Thus, estimate (3.9) implies that

$$
\begin{aligned}
& \mathbb{H}_{\varepsilon}\left(\left.\mathcal{A}\right|_{B_{0}^{R \ln 1 / \varepsilon}}, W^{2, q}\left(B_{0}^{R \ln 1 / \varepsilon}\right)\right) \leq \\
& \quad \leq \mathbb{H}_{\varepsilon /(C R \ln 1 / \varepsilon)^{n / q}}\left(\mathcal{A}, W_{b}^{2, q}\left(B_{0}^{R \ln 1 / \varepsilon}\right)\right) \leq C(R)\left(\ln \frac{1}{\varepsilon}\right)^{n+1}
\end{aligned}
$$

where $\ln \ln 1 / \varepsilon$ is majoranted by $\ln 1 / \varepsilon$. Corollary 3.5 is proved.

Corollary 3.6. Let the assumptions of previous corollary hold and let $\mathcal{K}$ be the same as in Theorem 3.1. Then, the following estimate is valid, for every $R>0$ :

$$
\mathbb{H}_{\mathcal{E}}\left(\mathcal{K}, L^{\infty}\left([0, R \ln 1 / \varepsilon], W_{b}^{2, q}\left(B_{0}^{R \ln 1 / \varepsilon}\right)\right) \leq C(R)\left(\ln \frac{1}{\varepsilon}\right)^{n+1}\right.
$$

Proof. Indeed, estimate (2.42), together with description (3.2), imply that, for every $T \geq 0$

$$
\mathbb{H}_{\mathcal{E}}\left(\mathcal{K}, L^{\infty}\left([0, T], W_{e^{-|x|}}^{2, q}\right)\right) \leq \mathbb{H}_{\varepsilon /\left(C e^{K T}\right)}\left(\mathcal{A}, W_{e^{-|x|}}^{2, q}\right)
$$


Inserting estimate (3.12) to this estimate and using obvious embedding $W_{b, e^{-2|x|}}^{2, q} \subset$ $W_{e^{-|x|}}^{2, q}$, we have

$$
\mathbb{H}_{\varepsilon}\left(\mathcal{K}, L^{\infty}\left([0, R \ln 1 / \varepsilon], W_{b, e^{-2|x|}}^{2, q}\left(B_{0}^{R \ln 1 / \varepsilon}\right)\right) \leq C_{R}\left(\ln \frac{1}{\varepsilon}\right)^{n+1}\right.
$$

We now note that

$$
\left\|v, B_{0}^{R \ln 1 / \varepsilon}\right\|_{b, 2, q} \leq e^{2 R \ln 1 / \varepsilon}\left\|v, B_{0}^{R \ln 1 / \varepsilon}\right\|_{b, e^{-2|x|}, 2, q}=\varepsilon^{-2 R}\left\|v, B_{0}^{R \ln 1 / \varepsilon}\right\|_{b, e^{-2|x|}, 2, q}
$$

and, consequently

$$
\begin{aligned}
& \mathbb{H}_{\mathcal{\varepsilon}}\left(\mathcal{K}, L^{\infty}\left([0, R \ln 1 / \varepsilon], W_{b}^{2, q}\left(B_{0}^{R \ln 1 / \varepsilon}\right)\right) \leq\right. \\
& \quad \leq \mathbb{H}_{\mathcal{E}^{2 R+1}}\left(\mathcal{K}, L^{\infty}\left([0, R \ln 1 / \varepsilon], W_{b, e^{-2|x|}}^{2, q}\left(B_{0}^{R \ln 1 / \varepsilon}\right)\right) \leq C_{R}^{\prime}\left(\ln \frac{1}{\varepsilon}\right)^{n+1}\right.
\end{aligned}
$$

Corollary 3.6 is proved.

Remark 3.2. Since $L^{\infty} \subset W_{b}^{2, q}\left(\mathbb{R}^{n}\right)$, then estimate (3.17) implies, in particular, the following estimate:

$$
\mathbb{H}_{\varepsilon}\left(\mathcal{K}, L^{\infty}\left([0, R \ln 1 / \varepsilon] \times B_{0}^{R \ln 1 / \varepsilon}\right)\right) \leq C_{R}\left(\ln \frac{1}{\varepsilon}\right)^{n+1}
$$

Moreover, arguing as in the proof of Corollary 3.5 and using the invariantness of $\mathcal{K}$, we have

$$
\mathbb{H}_{\varepsilon}\left(\mathcal{K}, L_{e^{-M|t|-M|x|}}^{\infty}\left(\mathbb{R} \times \mathbb{R}^{n}\right)\right) \leq C_{M}\left(\ln \frac{1}{\varepsilon}\right)^{n+1} .
$$

\section{$\S 4$ QuANTIATATIVE CHARACTERISTICS FOR THE SPATIAL AND TEMPORAL DYNAMICS.}

In this Section, we introduce several characteristics for the dynamical system, generated by equation (2.1) which generalize the concept of topological entropy to the case of unbounded domains. For simplicity, we restrict ourselves to consider only the case of spatially homogeneous equation $(2.1)$ in $\Omega=\mathbb{R}^{n}$, i.e. we assume that

$$
L(x) \equiv L \in \mathbb{R}^{n}, \quad g(x) \equiv g \in \mathbb{R}^{n} .
$$

In this case, the attractor $\mathcal{A}$ of equation (2.1) possesses an additional structure, namely, the group $\left\{T_{h}, h \in \mathbb{R}^{n}\right\}$ of spatial shifts acts on it:

$$
T_{h} \mathcal{A}=\mathcal{A}, \quad\left(T_{h} u_{0}\right)(x):=u_{0}(x+h), \quad h \in \mathbb{R}^{n} .
$$

We recall that, by the definition of the attractor, the dynamical semigroup $\left\{S_{t}, t \geq\right.$ $0\}$ defined by (2.41) also acts on the attractor. Moreover, this semigroup evidently commutes with group (4.2) of spatial shifts:

$$
S_{t} \mathcal{A}=\mathcal{A}, \quad T_{h} S_{t}=S_{t} T_{h}, \quad \forall t \in \mathbb{R}_{+}, h \in \mathbb{R}^{n} .
$$


Thus, the extended $(n+1)$-parametrical semigroup $\left\{\mathbb{S}_{(t, h)}, t \in \mathbb{R}_{+}, h \in \mathbb{R}^{n}\right\}$ acts on the attractor:

$$
\mathbb{S}_{(t, h)} \mathcal{A}=\mathcal{A}, \quad\left(\mathbb{S}_{(t, h)} u_{0}\right)(x):=S_{t} u_{0}(x+h), \quad t \in \mathbb{R}_{+}, \quad h \in \mathbb{R}^{n} .
$$

Extended semigroup (4.4) can be interpreted as a dynamical system (with multidymensional 'time') acting on the phace space $\mathcal{A}$, and, consequently, can be studied from the dynamical point of view.

We first recall the definition of the topological entropy (see e.g. [25]) adopted to the case of multidimensional 'time'. To this end, we endow our attractor $\mathcal{A}$ by the metric of the space $L_{\phi}^{\infty}\left(\mathbb{R}^{n}\right)$, where the weight $\phi$ has exponential growth rate (see Section 1) and satisfies the following assumption:

$$
\lim _{|x| \rightarrow \infty} \phi(x)=0
$$

It is not difficult to verify that, due to the fact that $\mathcal{A}$ is bounded in $L^{\infty}\left(\mathbb{R}^{n}\right)$, the topologies induced on $\mathcal{A}$ by the embeddings $\mathcal{A} \subset L_{\phi}^{\infty}\left(\mathbb{R}^{n}\right)$ and $\mathcal{A} \subset L_{\text {loc }}^{\infty}\left(\mathbb{R}^{n}\right)$ coinside (see e.g. [43]). In particular, this topology is independent of the particular choice of the weight $\phi$. For every $R>0$, we define a new metric on the attractor $\mathcal{A}$ by the following expression:

$$
\mathrm{d}_{R, \phi}\left(u_{0}, v_{0}\right):=\sup _{(t, h) \in[0, R]^{n+1}}\left\|\mathbb{S}_{(t, h)} u_{0}-\mathbb{S}_{(t, h)} v_{0}\right\|_{L_{\phi}^{\infty}}
$$

Definition 4.1. The topological entropy of semigroup (4.4) is the following number:

$$
\widehat{h}_{n+1}(\mathcal{A}):=\lim _{\varepsilon \rightarrow 0} \limsup _{R \rightarrow \infty} \frac{1}{R^{n+1}} \mathbb{H}_{\varepsilon}\left(\mathcal{A}, \mathrm{d}_{R, \phi}\right)
$$

where the symbol $\mathbb{H}_{\mathcal{E}}\left(\mathcal{A}, \mathrm{d}_{R, \phi}\right)$ denotes the Kolmogorov's $\varepsilon$-entropy of the set $\mathcal{A}$ in the space generated by the metric $\mathrm{d}_{R, \phi}$ (obviously, $\mathbb{H}_{\mathcal{E}}\left(\mathcal{A}, \mathrm{d}_{R, \phi}\right)$ is a nondecreasing function of $\varepsilon$, so limit (4.7) exists).

It is well known (see e.g. [25]), that topological entropy (4.7) depends only on the topology in $\mathcal{A}$ and independent of the particular choice of the metric on it. In particular, quantity (4.7) is independent of $\phi$. Moreover, it is not difficult to verify (analogously to [43]) that

$$
\widehat{h}_{n+1}(\mathcal{A})=\lim _{\varepsilon \rightarrow 0} \limsup _{R \rightarrow \infty} \frac{1}{R^{n+1}} \mathbb{H}_{\varepsilon}\left(\mathcal{K}, L^{\infty}\left([0, R]^{n+1}\right)\right),
$$

where the set $\mathcal{K}$ is defined in Theorem 3.1.

Proposition 4.1. The following limit exists, for every $\varepsilon>0$ :

$$
\overline{\mathbb{H}}_{\mathcal{E}}(\mathcal{K}):=\lim _{\substack{R_{i} \rightarrow+\infty \\ i=1, ., n+1}} \frac{1}{R_{1} \cdots R_{n+1}} \mathbb{H}_{\mathcal{E}}\left(\mathcal{K}, L^{\infty}\left(\left[0, R_{1}\right] \times \cdots \times\left[0, R_{n+1}\right]\right)\right)
$$

Proof. Indeed, let

$$
\Phi\left(R_{1}, \cdots, R_{n+1}\right):=\mathbb{H}_{\varepsilon}\left(\mathcal{K}, L^{\infty}\left(\left[0, R_{1}\right] \times \cdots \times\left[0, R_{n+1}\right]\right)\right) .
$$


Then, it follows from the invariantness of $\mathcal{K}$ under the spatial and temporal shifts that this function is subadditive with respect to every arguments:

$$
\begin{aligned}
\Phi\left(R_{1}, \cdots, R_{i}^{\prime}+R_{i}^{\prime \prime}, \cdots, R_{n+1}\right) \leq \Phi\left(R_{1}, \cdots,\right. & \left.R_{i}^{\prime}, \cdots, R_{n+1}\right)+ \\
& +\Phi\left(R_{1}, \cdots, R_{i}^{\prime \prime}, \cdots, R_{n+1}\right)
\end{aligned}
$$

and, cosequently

$$
\lim _{\substack{R_{i} \rightarrow+\infty \\ i=1, \ldots, n+1}} \Phi\left(R_{1}, \cdots, R_{n+1}\right)=\inf _{\substack{R_{i}>0 \\ i=1, \ldots, n+1}} \Phi\left(R_{1}, \cdots, R_{n+1}\right)
$$

which finishes the proof of Proposition 4.1.

Corollary 4.1. The following expressions can be considered as the equivalent definitions of the topological entropy $\widehat{h}_{n+1}(\mathcal{A})$ :

$$
\begin{aligned}
\widehat{h}_{n+1}(\mathcal{A}) & =\lim _{\varepsilon \rightarrow 0} \lim _{R \rightarrow+\infty} \frac{1}{R^{n+1}} \mathbb{H}_{\varepsilon}\left(\mathcal{K}, L^{\infty}\left([0, R]^{n+1}\right)\right)= \\
& =\lim _{\varepsilon \rightarrow 0} \lim _{R \rightarrow+\infty} \frac{1}{(2 R)^{n}} \lim _{T \rightarrow+\infty} \frac{1}{T} \mathbb{H}_{\varepsilon}\left(\mathcal{K}, L^{\infty}\left([0, T], L^{\infty}\left([-R, R]^{n}\right)\right)\right) .
\end{aligned}
$$

We note that the expression in the right-hand side of (4.12) is equivalent to the so called topological entropy per unit volume introduced in [12]. Thus, relation (4.12) gives, in particular, the dynamical and geometrical interpretation of the topological entropy per unit volume.

We note that, a priori, expression (4.7) may be infinite. The following theorem shows that it is not the case in our situation.

Theorem 4.1. Let the assumptions of Theorem 3.1 hold and let $\Omega=\mathbb{R}^{n}$ and (4.1) be satisfied. Then, the topological entropy defined by (4.7) is finite:

$$
\widehat{h}_{n+1}(\mathcal{A}) \leq C<\infty \text {. }
$$

Proof. Indeed, due to subadditivity (4.11) and due to (3.20), for every $R>\ln 1 / \varepsilon$, we have

$$
\begin{aligned}
\mathbb{H}_{\varepsilon}\left(\mathcal{K}, L^{\infty}\left([0, R]^{n+1}\right)\right) \leq\left(\frac{R}{\ln 1 / \varepsilon}+1\right)^{n+1} \mathbb{H}_{\varepsilon} & \left(\mathcal{K}, L^{\infty}\left([0, \ln 1 / \varepsilon]^{n+1}\right)\right) \leq \\
& \leq C_{1} R^{n+1}+C_{2} R^{n}(\ln 1 / \varepsilon)^{n+1}
\end{aligned}
$$

and, consequently

$$
\widehat{h}_{n+1}(\mathcal{A}) \leq C_{1}
$$

Theorem 4.1 is proved.

Let us study now the analogues of the quantities $\widehat{h}_{n+1}(\mathcal{A})$ for the $k$-parametrical subsemigroups of the extended dynamical system $\left\{\mathbb{S}_{(t, h)}, t \geq 0, h \in \mathbb{R}^{n}\right\}$. Let $V_{k} \subset \mathbb{R}_{t} \times \mathbb{R}_{x}^{n}$ be an arbitrary $k$-dimensional hyperplane, then we consider the following subsemigroup of extended dynamical system (4.4):

$$
\mathbb{S}_{(t, h)}^{V_{k}}:=\left\{\mathbb{S}_{(t, h)},(t, h) \in V_{k} \cap\left(R_{+} \times \mathbb{R}^{n}\right)\right\}
$$


It is evident, that

$$
\mathbb{S}_{(t, h)}^{\mathbb{R}_{t}}=S_{t} \text { and } \mathbb{S}_{(t, h)}^{\mathbb{R}_{x}^{n}}=T_{h}
$$

Let us fix now the orthonormal basis $\left\{e_{1}, \cdots, e_{k}\right\}$ in $V_{k}$ in such way that $e_{i} \subset \mathbb{R}_{x}^{n}$, for $i=2, \cdots, k$, and the semiaxis $\left\{h e_{1}, h \in \mathbb{R}_{+}\right\} \in \mathbb{R}_{+} \times \mathbb{R}^{n}$ and, analogously to (4.6), define a new metric on $\mathcal{A}$ :

$$
\mathrm{d}_{R, V_{k}}\left(u_{0}, v_{0}\right):=\sup _{\substack{0 \leq l_{i} \leq R \\ i=1, \cdots, k}}\left\|\mathbb{S}_{\sum_{i} l_{i} e_{i}} u_{0}-\mathbb{S}_{\sum_{i} l_{i} e_{i}} v_{0}\right\|_{L_{e^{-|x|}}^{\infty}\left(\mathbb{R}^{n}\right)}
$$

Definition 4.2. The (modified) toplogical entropy of semigroup (4.12) is the following number:

$$
\widehat{h}_{k}^{V_{k}}(\mathcal{A}):=\limsup _{\varepsilon \rightarrow 0}\left(\ln \frac{1}{\varepsilon}\right)^{k-n-1} \limsup _{R \rightarrow \infty} \frac{1}{R^{k}} \mathbb{H}_{\varepsilon}\left(\mathcal{A}, \mathrm{d}_{R, V_{k}}\right)
$$

Remark 4.2. We note that, in the case $k=n+1$, quantity (4.15), coinsides with expression (4.7) and, for $k \neq n+1$, it differs from the standard Definition 4.1 of the topological entropy by the factor $(\ln 1 / \varepsilon)^{k-n-1}$, which is introduced in order to provide the finiteness of limit (4.15) (see Theorem 4.2 below and the examples in Sections 5 and 7 ).

We also note that, although we have defined quantities (4.15) using a special basis in the space $V_{k}$, it is not difficult to verify that these quantities are, in fact, independent on the concrete choice of the basis and depend only on the subspace $V_{k}$.

It is also worth to emphasize that, in contrast to the case of $k=n+1$, quantities (4.15) are not topological invariants, but only Liptschitz continuious invariants and depend, consequently, on the particular choice of the metric, if $k<n+1$ (it is reasonable from many points of view to fix the exponentially decaying metric $L_{e^{-|x|}}^{\infty}$ on the attractor $\mathcal{A}$ (as it is implicitly done in Definition 4.2)).

Moroeover, although quantities (4.15) are not invariant under the Hölder continuous homeomorphisms, but, obviously

$$
\widehat{h}_{k}^{V_{k}}(F(\mathcal{A})) \leq \alpha^{k-n-1} \widehat{h}_{k}^{V_{k}}(\mathcal{A}),
$$

where $0<\alpha \leq 1$ is the Hölder constant of the homeomorphism $F$. Consequently, the property of the (modified) toipological entropy to be equal zero or to be strictly positive preserves under the Hölder continuous homeomorphisms, in particular, it preserves under the replacing of the initial weighted metric of $L_{e^{-|x|}}^{\infty}$ on the attractor by the metric of $L_{e^{-\mu\left|x-x_{0}\right|}}^{\infty}$, for every $\mu>0$ and $x_{0} \in \mathbb{R}^{n}$.

Remark 4.3. The most natural choices of the hyperplane $V_{k}$ are the following:

$$
\widehat{h}_{s p}(\mathcal{A}):=\widehat{h}_{n}^{\mathbb{R}_{x}^{n}}(\mathcal{A}) \text { and } \widehat{h}_{t}(\mathcal{A}):=\widehat{h}_{1}^{\mathbb{R}_{t}}(\mathcal{A}),
$$

which are responsible to the spatial and temporal complexity of the dynamics respectively. The fact that these quantitatives are striclty positive for a sufficiently large class of equations (2.1) will be verified in Sections 5 and 7 .

Let us formulate now the analogue of Proposition 4.1 and Corollary 4.1 for the entropies introduced in (4.15). 
Proposition 4.2. Let $W_{k}:=V_{k}^{\perp} \cap \mathbb{R}_{x}^{n}$, where $V_{k}^{\perp}$ stands for the orthogonal complement of the space $V_{k}$, let $x=\left(x^{\prime \prime}, x^{\prime}\right)$ correspond to the decomposition $\mathbb{R}_{x}^{n}=\left(V_{k} \cap \mathbb{R}_{x}^{n}\right) \times W_{k}$ and $e_{1}=e_{1}^{\prime}+e_{1}^{\prime \prime}$, where $e_{1}^{\prime} \in W_{k}, e_{1}^{\prime \prime} \in \mathbb{R}_{t}$. Then, quantity (4.15) can be defined in the following equivalent way:

$$
\widehat{h}_{k}^{V_{k}}(\mathcal{A})=\limsup _{\varepsilon \rightarrow 0}\left(\ln \frac{1}{\varepsilon}\right)^{k-n-1} \lim _{R \rightarrow \infty} \frac{1}{R^{k}} \mathbb{H}_{\varepsilon}\left(\mathcal{K}, L_{e^{-\left|x^{\prime}-t e_{1}^{\prime}\right|}}^{\infty}\left(V_{k}(R) \times W_{k}\right)\right)
$$

where $V_{k}(R):=\left[0, R e_{1}^{\prime \prime}\right] \times\left(\left[0, R e_{2}\right] \times \cdots \times\left[0, R e_{k}\right]\right)$ (if $e_{1} \in \mathbb{R}_{x}^{n}$, then $t=0$ and the set $\mathcal{K}$ should be replaced by $\mathcal{A}$, according to relation (3.2) and the vector $e_{1}^{\prime \prime}$ in the definition of $V_{k}(R)$ should be replaced by $\left.e_{1} \in \mathbb{R}_{x}^{n}\right)$.

Proof. We consider below only the most complicated case $e_{1}^{\prime \prime} \neq 0$ (the case $e_{1}^{\prime \prime}=0$ and, consequently, $V_{k} \subset \mathbb{R}_{x}^{n}$ is analogous, but even slightly more simple).

It follows from the definition of the set $\mathcal{K}$ and from (4.14) and (4.15) that

$$
\widehat{h}_{k}^{V_{k}}(\mathcal{A})=\limsup _{\varepsilon \rightarrow 0}\left(\ln \frac{1}{\varepsilon}\right)^{k-n+1} \limsup _{R \rightarrow+\infty} \frac{1}{R^{k}} \mathbb{H}_{\varepsilon}\left(\mathcal{K}, L_{\phi_{R}}^{\infty}\left(\left[0, R e_{1}^{\prime \prime}\right] \times \mathbb{R}_{x}^{n}\right)\right)
$$

where

$$
\phi_{R}(t, x):=\sup _{l^{\prime \prime} \in[0, R]^{k-1}} e^{-\left|\left(x^{\prime}-t e_{1}^{\prime}, x^{\prime \prime}-l^{\prime \prime}\right)\right|} .
$$

We note that $\phi_{R}(t, x) \equiv e^{-\left|x^{\prime}-t e_{1}^{\prime}\right|}$, if $x^{\prime \prime} \in[0, R]^{k-1}$ and

$$
\phi_{R}(t, x) \leq e^{-\left|x^{\prime}-t e_{1}^{\prime}\right|} e^{-\alpha \operatorname{dist}\left(x^{\prime \prime},[0, R]^{k-1}\right)},
$$

with an appropriate $\alpha>0$, if $x^{\prime \prime} \notin[0, R]^{k-1}$, consequently, there exists a constant $K>0$ which is independent of $R$ such that

$$
\begin{aligned}
\mathbb{H}_{\varepsilon}\left(\mathcal{K}, L_{e^{-\left|x^{\prime}-t e_{1}^{\prime}\right|}}^{\infty}\left(V_{k}(R)\right.\right. & \left.\left.\times W_{k}\right)\right) \leq \mathbb{H}_{\varepsilon}\left(\mathcal{K}, L_{\phi_{R}}^{\infty}\left(\left[0, R e_{1}^{\prime \prime}\right] \times \mathbb{R}_{x}^{n}\right) \leq\right. \\
\leq & \mathbb{H}_{\varepsilon}\left(\mathcal{K}, L_{e^{-\left|x^{\prime}-t e_{1}^{\prime}\right|}}^{\infty}\left(V_{k}(R+2 K \ln 1 / \varepsilon) \times W_{k}\right)\right)
\end{aligned}
$$

Multiplying (4.22) by $R^{-k}$ and passing to the limit $R \rightarrow \infty$, we derive (4.18). The existence of the limit as $R \rightarrow+\infty$ follows (as in Proposition 4.1) from the obvious subadditivity of the function

$$
\begin{aligned}
& \Phi_{k}\left(R_{1}, \cdots, R_{k}\right):= \\
& \quad=\mathbb{H}_{\mathcal{E}}\left(\mathcal{K}, L_{e^{-\left|x^{\prime}-t e_{1}^{\prime}\right|}}^{\infty}\left(\left[0, R_{1} e_{1}^{\prime \prime}\right] \times\left[0, R_{2} e_{2}\right] \times \cdots \times\left[0, R_{k} e_{k}\right] \times W_{k}\right)\right) .
\end{aligned}
$$

Proposition 4.2 is proved.

We recall that, analogously to Definition 4.1 , the $k$-dimensional topological entropy introduced in (4.15) also, a priori, may be infinite. The following analogue of Theorem 4.1 shows that it is not the case in our situation. 
Theorem 4.2. Let the assumptions of Theorem 3.1 hold and let, in addition, $\Omega=$ $\mathbb{R}^{n}$ and (4.1) be satisfied. Then, for every $k \in[0, \cdots, n+1]$ and for every hyperplane $V_{k} \in \mathbb{R}_{t} \times \mathbb{R}_{x}^{n}$, the corresponding (modified) topological entropy is finite:

$$
\widehat{h}_{k}^{V_{k}}(\mathcal{A}) \leq C<\infty \text {. }
$$

Proof. Let us verify (4.24) only for the most complicated case $e_{1}^{\prime \prime} \neq 0$ (the case $e_{1}^{\prime \prime}=0$ can be considered analogously). Using the subadditivity of function (4.23), we derive that, for $R>>\ln 1 / \varepsilon$, the following estimate is valid:

$$
\begin{aligned}
\mathbb{H}_{\varepsilon}\left(\mathcal{K}, L_{e^{-\left|x^{\prime}-t e_{1}^{\prime}\right|}}^{\infty}\right. & \left.\left(V_{k}(R) \times W_{k}\right)\right) \leq \\
& \leq\left(\frac{R}{\ln 1 / \varepsilon}+1\right)^{k} \mathbb{H}_{\varepsilon}\left(\mathcal{K}, L_{e^{-\left|x^{\prime}-t e_{1}^{\prime}\right|}}^{\infty}\left(V_{k}(\ln 1 / \varepsilon) \times W_{k}\right)\right)
\end{aligned}
$$

We now note that, if $t \in[0, \ln 1 / \varepsilon]$, then

$$
\varepsilon e^{-\left|x^{\prime}\right|} \leq e^{-\left|x^{\prime}-t e_{1}^{\prime}\right|} \leq \frac{1}{\varepsilon} e^{-\left|x^{\prime}\right|}
$$

and, consequently, (due to the boundedness of $\mathcal{K}$ in $L^{\infty}$ and estimate (3.20))

$$
\begin{aligned}
\mathbb{H}_{\varepsilon}\left(\mathcal{K}, L_{e^{-\left|x^{\prime}-t e_{1}^{\prime}\right|}}^{\infty}\left(V_{k}(\ln 1 / \varepsilon) \times W_{k}\right)\right) \leq \mathbb{H}_{\varepsilon^{2}}\left(\mathcal{K}, L_{e^{-\left|x^{\prime}\right|}}^{\infty}\left(V_{k}(\ln 1 / \varepsilon) \times W_{k}\right)\right) \leq \\
\leq \mathbb{H}_{\varepsilon^{2}}\left(\mathcal{K}, L^{\infty}\left(V_{k}(\ln 1 / \varepsilon) \times[0, K \ln 1 / \varepsilon]^{n+1-k}\right)\right) \leq \\
\leq \mathbb{H}_{\varepsilon^{2}}\left(\mathcal{K}, L^{\infty}\left(\left[0, K_{1} \ln \frac{1}{\varepsilon^{2}}\right]^{n+1}\right)\right) \leq C\left(\ln \frac{1}{\varepsilon}\right)^{n+1}
\end{aligned}
$$

Inserting the obtained estimate to (4.25), we have

$$
\mathbb{H}_{\mathcal{E}}\left(\mathcal{K}, L_{e^{-\left|x^{\prime}-t e_{1}^{\prime}\right|}}^{\infty}\left(V_{k}(R) \times W_{k}\right)\right) \leq C_{1} R^{k}\left(\ln \frac{1}{\varepsilon}\right)^{n+1-k}+C_{2} R^{k-1}\left(\ln \frac{1}{\varepsilon}\right)^{n+1}
$$

Inserting this estimate to (4.18), we derive the finiteness of $\widehat{h}_{k}^{V_{k}}(\mathcal{A})$ and finish the proof of Theorem 4.2.

The following theorem clarifies the relations between the topological entropies which correspond to different $k$.

Theorem 4.3. Let the assumptions of Theorem 4.1 hold and let the toplogical entropy which corresponds to some $k$-dimensional hyperplane $V_{k}(0<k \leq n+1)$ be strictly positive:

$$
\widehat{h}_{k}^{V_{k}}(\mathcal{A})>0 .
$$

Then, for every $k^{\prime}<k$ and every $k^{\prime}$-dimensional hyperplane $V_{k^{\prime}}^{\prime} \subset V_{k}$, the corresponding topological entropy is also strictly positive:

$$
\widehat{h}_{k^{\prime}}^{V_{k^{\prime}}^{\prime}}(\mathcal{A})>0
$$


Proof. We first note that it is sufficient to consider only the case $k^{\prime}=k-1$, i.e. $V_{k}=V_{k^{\prime}} \times\{\mathbb{R} e\}$, where $e \in \mathbb{R}_{t} \times \mathbb{R}_{x}^{n}$. There are two main possibilities (the other ones can be easily reduced to them):

1. $V_{k^{\prime}} \subset \mathbb{R}_{x}^{n}$, but $V_{k}$ contains the temporal directions $\left(e \notin \mathbb{R}_{x}^{n}\right)$.

2. We add the spatial direction $e \in \mathbb{R}_{x}^{n}$.

Let us consider the first case. We fix the orthonormal basis $\left\{e_{1}, \cdots, e_{k}\right\}$ in $V_{k}$ in such way that $\left\{e_{2}, \cdots, e_{k}\right\}$ is an orthonormal basis in $V_{k^{\prime}} \subset \mathbb{R}_{x}^{n}$. Let us assume now that the assertion of the theorem is wrong and

$$
\widehat{h}_{k-1}^{V_{k^{\prime}}}(\mathcal{A})=0 .
$$

Then, according to Proposition 4.2

$$
\lim _{\varepsilon \rightarrow 0}\left(\ln \frac{1}{\varepsilon}\right)^{k-n-2} \limsup _{R \rightarrow \infty} \frac{1}{R^{k-1}} \mathbb{H}_{\varepsilon}\left(\mathcal{A}, L_{\phi_{R}}^{\infty}\left(\mathbb{R}^{n}\right)\right)=0
$$

where $\phi_{R}(x):=\sup _{l^{\prime \prime} \in[0, R]^{k-1}} e^{-\mid\left(x^{\prime}, x^{\prime \prime}-l^{\prime \prime} \mid\right.}\left(x=x^{\prime}+x^{\prime \prime}, x^{\prime} \in V_{k-1}^{\perp}, x^{\prime \prime} \in V_{k-1}\right)$.

We note that the weight $\phi_{R}(x)$ has exponential growth rate $\mu=1$, moreover this weight satisfies (1.1) with the constant $C_{\phi_{R}} \leq C$ where $C$ is independent of $R$. Consequently, it follows from estimates (2.43) and (1.19) that, for every $u_{0}, v_{0} \in \mathcal{A}$, the following estimate is valid:

$$
\left\|S_{1} u_{0}-S_{1} v_{0}\right\|_{W_{b, \phi_{R}^{q}}^{2, q}\left(\mathbb{R}^{n}\right)} \leq C_{1}\left\|u_{0}-v_{0}\right\|_{L_{b, \phi_{R}^{q}}^{q}\left(\mathbb{R}^{n}\right)} \leq C_{2}\left\|u_{0}-v_{0}\right\|_{L_{\phi_{R}}^{\infty}\left(\mathbb{R}^{n}\right)},
$$

where the constants $C_{i}$ are independent of $R$ and $u_{0}, v_{0} \in \mathcal{A}$ (see [39] and [43]). Estimate (4.31), together with the invariantness of the attractor, imply that

$$
\mathbb{H}_{\varepsilon / C_{2}}\left(\mathcal{A}, W_{b, \phi_{R}^{q}}^{2, q}\left(\mathbb{R}^{n}\right)\right) \leq \mathbb{H}_{\varepsilon}\left(\mathcal{A}, L_{\phi_{R}}^{\infty}\left(\mathbb{R}^{n}\right)\right) .
$$

Arguing analogously, but using estimate (2.42) with $T=\ln 1 / \varepsilon$ instead of (2.43), we have

$$
\begin{aligned}
\mathbb{H}_{\varepsilon}(\mathcal{K}, & \left.L_{\phi_{R}}^{\infty}\left([0, \ln 1 / \varepsilon] \times \mathbb{R}^{n}\right)\right) \leq \\
& \left.\leq \mathbb{H}_{\varepsilon / C_{1}}\left(\mathcal{K}, L^{\infty}\left([0, \ln 1 / \varepsilon], W_{b, \phi_{R}^{q}}^{2, q} \mathbb{R}^{n}\right)\right)\right) \leq \mathbb{H}_{\varepsilon^{m} / C_{2}}\left(\mathcal{A}, L_{\phi_{R}}^{\infty}\left(\mathbb{R}^{n}\right)\right)
\end{aligned}
$$

where $m:=K+1$ is independent of $\varepsilon$ and $\phi_{R}$. Using inequality (4.26), we deduce from (4.33) that

$$
\begin{aligned}
& \mathbb{H}_{\varepsilon}\left(\mathcal{K}, L_{e^{-\left|x^{\prime}-t e_{1}^{\prime}\right|}}^{\infty}\left(\left[0,(\ln 1 / \varepsilon) e_{1}^{\prime \prime}\right] \times V_{k-1}^{\prime}(R) \times W_{k-1}\right)\right) \leq \\
& \leq \mathbb{H}_{\varepsilon^{2}}\left(\mathcal{K}, L_{e^{-\left|x^{\prime}\right|}}^{\infty}\left(\left[0,(\ln 1 / \varepsilon) e_{1}^{\prime \prime}\right] \times V_{k-1}^{\prime}(R) \times W_{k-1}\right)\right) \leq \\
& \quad \leq \mathbb{H}_{\varepsilon^{2}}\left(\mathcal{K}, L_{\phi_{R}}^{\infty}\left([0, \ln 1 / \varepsilon] \times \mathbb{R}^{n}\right)\right) \leq \mathbb{H}_{\varepsilon^{2 m} / C_{3}}\left(\mathcal{A}, L_{\phi_{R}}^{\infty}\left(\mathbb{R}^{n}\right)\right)
\end{aligned}
$$

where $V_{k-1}^{\prime}(R):=\left[0, R e_{2}\right] \times \cdots \times\left[0, R e_{k}\right]$.

Estimates (4.30) and (4.34) imply that, for every $\mu>0$, there is $\varepsilon_{0}$ such that, for every $\varepsilon>\varepsilon_{0}$

$$
\lim _{R \rightarrow \infty} \frac{1}{R^{k-1}} \mathbb{H}_{\varepsilon}\left(\mathcal{K}, L_{e^{-\left|x^{\prime}-t e_{1}^{\prime}\right|}}^{\infty}\left(\left[0, \ln \frac{1}{\varepsilon} e_{1}^{\prime \prime}\right] \times V_{k-1}^{\prime}(R) \times W_{k-1}\right)\right) \leq \mu\left(\ln \frac{1}{\varepsilon}\right)^{n+2-k} .
$$


Using the subadditivity of function (4.23), we derive from the last estimate that

$$
\begin{aligned}
\lim _{T \rightarrow \infty} \frac{1}{T} \lim _{R \rightarrow \infty} \frac{1}{R^{k-1}} \mathbb{H}_{\varepsilon}\left(\mathcal{K}, L_{e^{-\left|x^{\prime}-t e_{1}^{\prime}\right|}}^{\infty}\left(\left[0, T e_{1}^{\prime \prime}\right] \times V_{k-1}^{\prime}(R) \times W_{k-1}\right)\right) & \\
& \leq \mu\left(\ln \frac{1}{\varepsilon}\right)^{n+1-k}
\end{aligned}
$$

and, consequently

$$
\begin{aligned}
\widehat{h}_{k}^{V_{k}}(\mathcal{A})=\limsup _{\varepsilon \rightarrow 0}\left(\ln \frac{1}{\varepsilon}\right)^{k-n-1} & \lim _{T \rightarrow \infty} \frac{1}{T} \lim _{R \rightarrow \infty} \frac{1}{R^{k-1}} \mathbb{H}_{\varepsilon}(\mathcal{K}, \\
& \left.L_{e^{-\left|x^{\prime}-t e_{1}^{\prime}\right|}}\left(\left[0, T e_{1}^{\prime \prime}\right] \times V_{k-1}^{\prime}(R) \times W_{k-1}\right)\right)=0
\end{aligned}
$$

which contradicts to assumption (4.27).

Let us now consider the second case and assume again that the assertion of the theorem is wrong, i.e., (4.29) is true. We fix the orthonormal basis $\left\{e_{1}, \cdots, e_{k}\right\}$ in $V_{k}$ in such way that $e_{i} \in \mathbb{R}_{x}^{n}$, for $i=2, \cdots, k$, and $\left\{e_{1}, \cdots, e_{k-1}\right\}$ be the orthonormal basis in $V_{k-1}$. Let us assume also that $e_{1}^{\prime \prime} \neq 0$ (the other case $e_{1}^{\prime \prime}=0$ is completely analogous). Let $x=\left(x^{\prime \prime}, x^{\prime}, y\right)$ be the decomposition of $x \in \mathbb{R}^{n}$ which corresponds to the decomposition $\mathbb{R}_{x}^{n}=\left(V_{k-1} \cap \mathbb{R}_{x}^{n}\right) \oplus W_{k} \oplus\left\{\mathbb{R}_{k}\right\}$. Then, using Proposition 4.2 and the fact that $W_{k-1}=W_{k} \oplus\left\{\mathbb{R} e_{k}\right\}$, we have

$$
\begin{aligned}
\lim _{\varepsilon \rightarrow 0}\left(\ln \frac{1}{\varepsilon}\right)^{k-n-2} \limsup _{R \rightarrow \infty} \frac{1}{R^{k-1}} \mathbb{H}_{\varepsilon}(\mathcal{K}, \\
L_{\left.e^{-\left|\left(x^{\prime}-t e_{1}^{\prime}, y\right)\right|}\left(V_{k-1}(R) \times W_{k-1}\right)\right)=0 .}
\end{aligned}
$$

Using the obvious inequality

$$
e^{-\left|\left(x^{\prime}-t e_{1}^{\prime}, y\right)\right|} \geq \varepsilon e^{-\left|x^{\prime}-t e_{1}^{\prime}\right|} \text { for }|y| \leq \ln 1 / \varepsilon,
$$

we derive from (4.35) that

$$
\begin{aligned}
\lim _{\varepsilon \rightarrow 0}\left(\ln \frac{1}{\varepsilon}\right)^{k-n-2} \limsup _{R \rightarrow \infty} & \frac{1}{R^{k-1}} \mathbb{H}_{\varepsilon}(\mathcal{K}, \\
& \left.L_{e^{-\left|x^{\prime}-t e_{1}^{\prime \prime}\right|}}^{\infty}\left(V_{k-1}(R) \times\left[0, e_{k} \ln \frac{1}{\varepsilon}\right] \times W_{k}\right)\right)=0 .
\end{aligned}
$$

Arguing now as in the end of the proof for the first case (after obtaining estimate (4.34)) and using the subadditivity of function (4.23), we derive that $\widehat{h}_{k}^{V_{k}}(\mathcal{A})=0$ which contradicts to (4.27) and finishes the proof of Theorem 4.3.

Remark 4.4. We have proved, in a fact, a slightly more strong result, namely, we have obtained the following estimate:

$$
\widehat{h}_{k}^{V_{k}}(\mathcal{A}) \leq L \widehat{h}_{k-1}^{V_{k-1}}(\mathcal{A}), \quad V_{k-1} \subset V_{k},
$$

for the appropriate constant $L$ which is independent of $k$ and $V_{k}$. This result can be considered as a generalization of the well known relation between the fractal dimension and the topological entropy for the Lipschitz continuous dynamical sytems with one dimensional 'time':

$$
h_{\text {top }}(\mathcal{A}) \leq L \operatorname{dim}_{F}(\mathcal{A})
$$

Indeed, for one dimensional 'time' $(n=0)$, we have $\widehat{h}_{0}(\mathcal{A})=\operatorname{dim}_{F}(\mathcal{A})$ and $\widehat{h}_{1}(\mathcal{A})=$ $h_{\text {top }}(\mathcal{A})$. 
Corollary 4.2. Let the assumptions of Theorem 4.1 hold and let, in addition, topological entropy (4.8) is strictly positive. Then, for every $k \in[0, \cdots, n+1]$ and for every $k$-dimensional hyperplane $V_{k}$, the corresponding topological entropy is also strictly positive: $\widehat{h}_{k}^{V_{k}}(\mathcal{A})>0$.

On the other hand, if the 0-dimensional entropy is equal to zero, i.e.

$$
\lim _{\varepsilon \rightarrow 0}\left(\ln \frac{1}{\varepsilon}\right)^{-n-1} \mathbb{H}_{\varepsilon}\left(\mathcal{A}, L^{\infty}\left(B_{0}^{\ln 1 / \varepsilon}\right)\right)=0,
$$

then all the entropies, defined by (4.14) are equal to zero.

Remark 4.5. It is possible to consider the spatial and temporal dynamics from the unified point of view. Indeed, let us endow the set $\mathcal{K}$, defined in Theorem 3.1 by the topology of the space $L_{e^{-|(t, x)|}}^{\infty}\left(\mathbb{R}^{n+1}\right)$ and consider the $(n+1)$-parametrical semigroup of spatial and temporal shifts $\left\{\mathbb{T}_{(s, h)}, s \in \mathbb{R}_{+}, h \in \mathbb{R}^{n}\right\}$ acting in this space. Then, by definition, the set $\mathcal{K}$ is strictly invariant under this semigroup:

$$
\mathbb{T}_{(s, h)} \mathcal{K}=\mathcal{K}, \quad\left(\mathbb{T}_{(s, h)}\right) u(t, x):=u(t+s, x+h), \quad s \in \mathbb{R}_{+}, \quad h \in \mathbb{R}^{n}
$$

and, consequently, we may define all quantities (4.15) for semigroup (4.39) as well. We denote them by $\widehat{h}_{k}^{V_{k}}(\mathcal{K})$. We claim that

$$
C_{1} \widehat{h}_{k}^{V_{k}}(\mathcal{K}) \leq \widehat{h}_{k}^{V_{k}}(\mathcal{A}) \leq C_{2} \widehat{h}_{k}^{V_{k}}(\mathcal{K})
$$

Indeed, let $\mathcal{K}^{+}:=\left.\mathcal{K}\right|_{t>0}$, then, according to (2.42), we have the Liptschitz continuous isomorphism between the sets

$$
\left(\mathcal{A}, W_{b, e^{-q|x|}}^{2, q}\left(\mathbb{R}^{n}\right)\right) \rightarrow\left(\mathcal{K}^{+}, L_{e^{-K|t|}}^{\infty}\left(\mathbb{R}_{+}, W_{b, e^{-q|x|}}^{2, q}\left(\mathbb{R}^{n}\right)\right)\right)
$$

which is realized by the solving operator of problem (2.1) (if $K$ is large enough, see also (4.31) and [43]). We also note that, due to (4.31), the entropies of $\mathcal{A}$ computed in the $L_{e^{-|x|}}^{\infty}$-metric coinsides with the ones computed in the metric of the left hand side of (4.41). Therefore, the topological entropies of $\mathcal{K}^{+}$computed in the metric of the right-hand side of (4.41) coinsides with the corresponding topological entropies of $\mathcal{A}$. Using now estimates (4.16) for estimating the entropies in the unified metric $e^{-|(t, x)|}$, we easily derive the following analogue of estimates (4.40):

$$
C_{1} \widehat{h}_{k}^{V_{k}}\left(\mathcal{K}^{+}\right) \leq \widehat{h}_{k}^{V_{k}}(\mathcal{A}) \leq C_{2} \widehat{h}_{k}^{V_{k}}\left(\mathcal{K}^{+}\right)
$$

Arguing as in the proof of Theorem 4.3, we derive that

$$
\widehat{h}_{k}^{V_{k}}\left(\mathcal{K}^{+}\right) \leq \widehat{h}_{k}^{V_{k}}(\mathcal{K}) \leq C_{3} \widehat{h}_{k}^{V_{k}}\left(\mathcal{K}^{+}\right)
$$

which implies estimate (4.40).

\section{$\S 5$ LOWER BOUNDS OF THE $\varepsilon$-ENTROPY AND SPATIAL CHAOS.}

In this Section, following [39] and [43], we derive the lower bounds for the Kolmogorov's $\varepsilon$-entropy of the attractor $\mathcal{A}$ of spatially homogeneous equation (2.1) in 
$\Omega=\mathbb{R}^{n}$ (assumptions (4.1) are assumed to be satisfied) and obtain a topological description of the spatial complexity of this attractor.

We first note that it follows from conditions (2.3) that equation (2.1) possesses at least one spatially homogeneous equilibrium $z_{0} \in \mathbb{R}^{n}, f\left(z_{0}\right)+\lambda_{0} z_{0}=g$. Without loss of generality, we may assume that $z_{0} \equiv 0(f(0)=g=0)$ and, consequently, equation (2.1) has the following view:

$$
\partial_{t} u=a \Delta_{x} u-\left(L, \nabla_{x}\right) u+B u-\bar{f}(u), \quad \bar{f}(0)=\bar{f}^{\prime}(0)=0,
$$

where $B:=-\lambda_{0} u-f^{\prime}(0) \in \mathcal{L}\left(\mathbb{R}^{k}, \mathbb{R}^{k}\right)$ and $\bar{f}(u):=f(u)-f^{\prime}(0) u$. Our main assumption is the following: the equilibrium $z_{0}=0$ is exponentially unstable, i.e.

$$
\sigma\left(a \Delta_{x}-\left(L, \nabla_{x}\right)+B\right) \cap\{\operatorname{Re} z>0\} \neq \varnothing,
$$

where $\sigma(T)$ denotes the spectrum of the operator $T$.

In order to formulate the result on the infinite dimensional unstable manifold for equation (5.1), we need the following classical spaces (see e.g. [27]).

Definition 5.1. We denote by $\mathbb{B}_{\sigma}\left(\mathbb{R}^{n}\right)=\mathbb{B}_{\sigma}\left(\mathbb{R}^{n}, \mathbb{C}\right)$ the subspace of $L^{\infty}\left(\mathbb{R}^{n}, \mathbb{C}\right)$ which consists of all functions $\phi$ the Fourier transform $\widehat{\phi}$ of which satisfies

$$
\operatorname{supp} \widehat{\phi} \subset[-\sigma, \sigma]^{n} \text {. }
$$

Analogously, we denote by $\mathbb{B}_{\xi, \sigma}\left(\mathbb{R}^{n}\right), \xi \in \mathbb{R}^{n}$ a slightly general class of functions which consists of functions $\phi$ the Fourier transform $\widehat{\phi}$ of which satisfies

$$
\operatorname{supp} \widehat{\phi} \subset \xi+[-\sigma, \sigma]^{n} \text {. }
$$

We recall that the space $\mathbb{B}_{\xi, \sigma}$ is isomorphic to $\mathbb{B}_{\sigma}$ and this homeomorphism is given by multiplication on the function $e^{i \xi \cdot x}$.

Theorem 5.1. Let the assumptions of Theorem 4.1 hold, equation (2.1) have the form (5.1), and assumption (5.2) be satisfied. Then, for every $N>>1$, there exist a positive number $\sigma>0$, a vector $\xi_{0} \in \mathbb{R}^{n}, \sigma<\left|\xi_{0}\right|$, a positive number $r=r(N)>0$ and a map

$$
\mathcal{U}_{0}: B\left(r, 0, \mathbb{B}_{\xi_{0}, \sigma}\right) \rightarrow \mathcal{A}, \quad T_{h} \circ \mathcal{U}_{0}=\mathcal{U}_{0} \circ T_{h},
$$

where $B(r, v, V)$ denotes the $r$-ball of the space $V$ centered at $v$, such that, for every $x_{0} \in \mathbb{R}^{n}$, the following estimates hold:

$$
\left\{\begin{array}{l}
\left\|\mathcal{U}_{0}\left(u_{1}\right)-\mathcal{U}_{0}\left(u_{2}\right), B_{x_{0}}^{1}\right\|_{2, q} \leq C_{N} \sup _{x \in \Omega} \frac{\left\|u_{1}-u_{2}, B_{x}^{1}\right\|_{0, \infty}}{\left(1+\left|x-x_{0}\right|^{2 N}\right)^{1 / 2}}, \\
\left\|u_{1}-u_{2}, B_{x_{0}}^{1}\right\|_{0, \infty} \leq C_{N} \sup _{x \in \Omega} \frac{\left\|\mathcal{U}_{0}\left(u_{1}\right)-\mathcal{U}_{0}\left(u_{2}\right), B_{x}^{1}\right\|_{2, q}}{\left(1+\left|x-x_{0}\right|^{2 N}\right)^{1 / 2}},
\end{array}\right.
$$

where the constant $C_{N}$ depends only on $N$ and is independednt of $x_{0}$.

Moreover, there exists a vector $\vec{l} \in \mathbb{R}^{k}$ and a linear operator $S: \mathbb{B}_{\xi_{0}, \sigma}\left(\mathbb{R}^{n}\right) \rightarrow$ $W_{b}^{2, q}\left(\mathbb{R}^{n}, \mathbb{R}^{k}\right)$ such that

$$
S\left(u_{0}\right) . \vec{l} \equiv \operatorname{Re} u_{0}, \quad \text { for every } u_{0} \in \mathbb{B}_{\xi_{0}, \sigma}\left(\mathbb{R}^{n}\right)
$$

and, for every $u_{0} \in B\left(r, 0, \mathbb{B}_{\xi_{0}, \sigma}\right)$, the following estimate hold:

$$
\left\|\mathcal{U}_{0}\left(u_{0}\right)-S\left(u_{0}\right)\right\|_{W_{b}^{2, q}\left(\mathbb{R}^{n}\right)} \leq C\left\|u_{0}\right\|_{L_{b}^{\infty}\left(\mathbb{R}^{n}\right)}^{2} .
$$

The detailed proof of this theorem is given in [43], for the case $L=0$. The general case $L \neq 0$ is completely analogous, so we omit the proof here (see also Section 7 where the proof of the analogous result will be given in a more complicated situation).

Estimate (5.8), together with the information about the $\varepsilon$-entropy in the spaces $\mathbb{B}_{\sigma}$ allows to obtain the lower bounds for the attractor of equation (2.1). 
Theorem 5.2. Let the assumptions of Theorem 5.1 hold. Then, the $\varepsilon$-entropy of the attractor $\mathcal{A}$ of this equation possesses the following estimate:

$$
\mathbb{H}_{\varepsilon}\left(\mathcal{A}, L^{\infty}\left(B_{0}^{R}\right)\right) \geq C R^{n} \ln \frac{1}{\varepsilon}, \quad \varepsilon \leq \varepsilon_{0}<1,
$$

which shows that (3.8) is sharp, if $R \gg \ln 1 / \varepsilon$ and for $R \sim \ln \frac{1}{\varepsilon}$.

Moreover, for every $\delta>0$, there exists a constant $C_{\delta}>0$ such that

$$
\mathbb{H}_{\varepsilon}\left(\mathcal{A}, L^{\infty}\left(B_{0}^{1}\right)\right) \geq C_{\delta}\left(\ln \frac{1}{\varepsilon}\right)^{n+1-\delta}, \quad \varepsilon \leq \varepsilon_{0}<1
$$

and, consequently, (3.8) is sharp for the case $R \ll \ln \frac{1}{\varepsilon}$ as well.

Proof. Let $u_{0}^{1}, u_{0}^{2} \in B\left(\mu, 0, \mathbb{B}_{\xi_{0}, \sigma}\right.$ ) and $\mu \leq r$ (where $r, \sigma, \xi_{0}$ are the same as in Theorem 5.1). Then, for every $R>0$

$$
\left\|\mathcal{U}_{0}\left(u_{0}^{1}\right)-\mathcal{U}_{0}\left(u_{0}^{2}\right)\right\|_{L^{\infty}\left(B_{0}^{R}\right)} \geq\left\|\operatorname{Re}\left(u_{0}^{1}-u_{0}^{2}\right)\right\|_{L^{\infty}\left(B_{0}^{R}\right)}-C \mu^{2},
$$

where $C$ is independent of $R$. Indeed, according to (5.8) and (5.7)

$$
\begin{aligned}
& \left\|\mathcal{U}_{0}\left(u_{0}^{1}\right)-\mathcal{U}_{0}\left(u_{0}^{2}\right)\right\|_{L^{\infty}\left(B_{0}^{R}\right)} \geq \\
& \geq\left\|S u_{0}^{1}-S u_{0}^{2}\right\|_{L^{\infty}\left(B_{0}^{R}\right)}-\left\|\mathcal{U}_{0}\left(u_{0}^{1}\right)-S u_{0}^{1}\right\|_{L^{\infty}\left(\mathbb{R}^{n}\right)}-\left\|\mathcal{U}_{0}\left(u_{0}^{2}\right)-S u_{0}^{2}\right\|_{L^{\infty}\left(\mathbb{R}^{n}\right)} \geq \\
& \geq\left\|S u_{0}^{1}-S u_{0}^{2}\right\|_{L^{\infty}\left(B_{0}^{R}\right)}-C_{1}\left(\left\|u_{0}^{1}\right\|_{\mathbb{B}_{\sigma, \xi_{0}}}^{2}+\left\|u_{0}^{2}\right\|_{\mathbb{B}_{\sigma, \xi_{0}}}^{2}\right) \geq \\
& \geq\left\|\operatorname{Re}\left(u_{0}^{1}-u_{0}^{2}\right)\right\|_{L^{\infty}\left(B_{0}^{R}\right)}-C \mu^{2} .
\end{aligned}
$$

Thus,

$$
\begin{array}{r}
\mathbb{H}_{\varepsilon / 4}\left(\mathcal{A}, L_{b}^{\infty}\left(B_{0}^{R}\right)\right) \geq \mathbb{H}_{\varepsilon}\left(B\left(\left(\frac{\varepsilon}{2 C}\right)^{1 / 2}, 0, \mathbb{B}_{\xi_{0}, \sigma}^{R e}\right), C_{b}\left(B_{0}^{R}\right)\right)= \\
=\mathbb{H}_{(2 C \varepsilon)^{1 / 2}}\left(B\left(1,0, \mathbb{B}_{\xi_{0}, \sigma}^{R e}\right), C_{b}\left(B_{0}^{R}\right)\right)
\end{array}
$$

where, by definition, $\mathbb{B}_{\xi_{0}, \sigma}^{\mathrm{Re}}\left(\mathbb{R}^{n}, \mathbb{R}\right):=\operatorname{Re} \mathbb{B}_{\xi_{0}, \sigma}\left(\mathbb{R}^{n}, \mathbb{C}\right)$. To complete the proof of the theorem, we need the following lemma.

Lemma 5.1. The $\varepsilon$-entropy of the unit balls in the spaces $\mathbb{B}_{\xi_{0}, \sigma}$ and $\mathbb{B}_{\xi_{0}, \sigma}^{\mathrm{Re}}$ possesses estimates (5.9) and $(5.10)$ (where $\mathcal{A}$ is replaced by $B\left(1,0, \mathbb{B}_{\xi_{0}, \sigma}\right)$ or $B\left(1,0, \mathbb{B}_{\xi_{0}, \sigma}^{\mathrm{Re}}\right)$ respectively).

The proof of the lemma is given, e.g. in [27] or [39] for the spaces $\mathbb{B}_{\xi_{0}, \sigma}$. The case of $\mathbb{B}_{\xi_{0}, \sigma}$ is completely analogous (see also [43]).

Estimating entropy in the right-hand side of (5.12) by Lemma 5.1, we immediately obtain estimates (5.9) and (5.10) for the $\varepsilon$-entropy of the attractor and finish the proof of Theorem 5.2.

Corollary 5.1. Let the assumptions of Theorem 5.1 hold. Then, the (modified) topological entropy $\widehat{h}_{s p}(\mathcal{A})$ (see (4.17)) is strictly positive:

$$
\begin{gathered}
\widehat{h}_{s p}(\mathcal{A})>0 \\
37
\end{gathered}
$$


and, consequently (due to Theorem 4.3), the topological entropies $\widehat{h}_{k}^{V_{k}}(\mathcal{A})$ are also strictly positive, for all $V_{k} \subset \mathbb{R}_{x}^{n}$.

Remark 5.1. It follows from (5.13) that the (classical) topological entropy of the group $\left\{T_{h}, h \in \mathbb{R}^{n}\right\}$ acting on the attractor (which is defined by (4.15) without the stabilizing factor $\left.(\ln 1 / \varepsilon)^{-1}\right)$ is infinite. The same is true for all its subsemigroups $\left\{T_{h}, h \in V_{k}\right\}$ if $V_{k} \subset \mathbb{R}_{x}^{n}$.

Theorem 5.1 allows also to obtain some topological description of the spatial complexity of the attractor $\mathcal{A}$.

Corollary 5.2. Let the assumptions of Theorem 5.1 hold and let

$$
\mathbb{K}:=B\left(r, 0, \mathbb{B}_{\xi_{0}, \sigma}\right) \text { endowed by the topology of } L_{\text {loc }}^{\infty}\left(\mathbb{R}^{n}\right),
$$

where $r, \sigma$ and $\xi_{0}$ be the same as in Theorem 5.1. Then, the map $\mathcal{U}_{0}$ realizes a homeomorphism

$$
\mathcal{U}_{0}:\left(\mathbb{K}, T_{h}\right) \rightarrow\left(\mathcal{U}_{0}(\mathbb{K}), T_{h}\right) \subset\left(\mathcal{A}, T_{h}\right)
$$

Moreover, this homeomorphism preserves the topological entropy $\widehat{h}_{s p}$

$$
0<\widehat{h}_{s p}(\mathbb{K})=\widehat{h}_{s p}\left(\mathcal{U}_{0}(\mathbb{K})\right) \leq \widehat{h}_{s p}(\mathcal{A})
$$

Proof. The fact that the map $\mathcal{U}_{0}$ is a homeomorphism in a local topology is an immediate corollary of estimates (5.6). The homeomorphism of 'dynamical systems' follows from the fact that $\mathcal{U}_{0}$ commutes with $T_{h}$ (see (5.5)). Relations (5.16) can be easily derived from Lemma 5.1 and from estimates (5.6) (see [43]). Corollary 5.2 is proved.

Thus, we have constructed the embedding of the model 'dynamical system' $\left(\mathbb{K}, T_{h}\right)$ to the spatial dynamics on the attractor. In order to clarify the chaotic nature of this model dynamical sytem, we restict ourselves to consider the discrete dynamics $\left\{T_{h}, h \in \alpha \mathbb{Z}^{n}\right\}$ for the appropriate $\alpha>0$. For this case, the description of the dynamics $\left(\mathbb{K}, T_{h}\right)$ can be essentially simplified.

Definition 5.2. Let $\mathbb{D}:=\{z \in \mathbb{C}:|z| \leq 1\}$ be a unit disk in $\mathbb{C}$. We set $\mathcal{M}:=\mathbb{D}^{\mathbb{Z}^{n}}$ and endow this space by the standard Tikhonov's topology. We define a group $\left\{\mathcal{T}_{l}, l \in \mathbb{Z}^{n}\right\}$ on $\mathcal{M}$ as follows:

$$
\left(\mathcal{T}_{l} v\right)(m):=v(l+m), \quad l, m \in \mathbb{Z}^{n}, \quad v \in \mathcal{M},
$$

where the space $\mathcal{M}$ is interpreted as a space of all functions $v: \mathbb{Z}^{n} \rightarrow \mathbb{D}$.

Propositrion 5.1. There exist a positive number $\alpha>0$ and a continuous map $\kappa: \mathcal{M} \rightarrow \mathbb{K}$ such that

$$
T_{\alpha l} \kappa\left(v_{0}\right)=\kappa\left(\mathcal{T}_{l} v_{0}\right), \quad l \in \mathbb{Z}^{n}, \quad v_{0} \in \mathcal{M}
$$

Moreover, for every weight function with polinomial growth rate $\mu<1$, the following estimate is valid:

$$
C_{1}\left\|v_{1}-v_{2}\right\|_{L_{\phi}^{\infty}\left(\mathbb{Z}^{n}\right)} \leq\left\|\kappa\left(v_{1}\right)-\kappa\left(v_{2}\right)\right\|_{L_{\phi}^{\infty}\left(\mathbb{R}^{n}\right)} \leq C_{2}\left\|v_{1}-v_{2}\right\|_{L_{\phi}^{\infty}\left(\mathbb{Z}^{n}\right)},
$$


for every $v_{1}, v_{2} \in \mathcal{M}$ (where the constants $C_{i}$ depend only on $\mu$ and $C_{\phi}$ from assumption (1.2)).

If we define the (modified) topological entropy for $\left(\mathcal{M}, \mathcal{T}_{l}\right)$ as follows:

$$
\widehat{h}_{s p}(\mathcal{M}):=\limsup _{\varepsilon \rightarrow 0}\left(\ln \frac{1}{\varepsilon}\right)^{-1} \lim _{T \rightarrow \infty} \frac{1}{T^{n}} \mathbb{H}_{\varepsilon}\left(\mathcal{M}, L^{\infty}\left([0, T]^{n}\right)\right)
$$

(compare with (4.15) and (4.18)), then the map $\kappa$ preserves the topological entropy (up to the multiplier $\left.\alpha^{n}\right)$ :

$$
0<\alpha^{-n} \widehat{h}_{s p}(\mathcal{M})=\widehat{h}_{s p}(\kappa(\mathcal{M})) \leq \widehat{h}_{s p}(\mathbb{K})
$$

The proof of Proposition 5.1 is based on the classical Kotelnikov-Cartrait interpolation formula (see [27]) for functions from the class $\mathbb{B}_{\sigma}$ and is given in [43].

Combining Theorem 5.2 and Proposition 5.1, we obtain the following result.

Theorem 5.3. Let the assumptions of Theorem 5.1 hold. Then, there exists a number $\alpha>0$ and a homeomorphic embedding

$$
\tau:\left(\mathcal{M}, \mathcal{T}_{l}\right) \rightarrow\left(\mathcal{A}, T_{\alpha l}\right), \quad l \in \mathbb{Z}^{n}
$$

which preserves the (modified) topological entropy

$$
0<\alpha^{-n} \widehat{h}_{s p}(\mathcal{M})=\widehat{h}_{s p}(\tau(\mathcal{M})) \leq \widehat{h}_{s p}(\mathcal{A})
$$

Indeed, it is sufficient to take $\tau:=\mathcal{U}_{0} \circ \kappa$.

Remark 5.2. The dynamical system $\left(\mathcal{M}, \mathcal{T}_{l}\right)$ can be considered as one of possible ways to generalize the symbolic dynamics (Bernoulli shifts, see e.g. [25]) to the case of infinite (and even continual) number of symbols. Indeed, considering the closed invariant subset $\mathcal{M}_{N} \subset \mathcal{M}$ which consists of functions $v: \mathbb{Z}^{n} \rightarrow\left\{a_{1}, \cdots, a_{N}\right\}$, where $a_{i} \in \mathbb{D}$ are different complex numbers, we obtain from (5.22) the embedding of the symbolic dynamics with finite number $N$ symbols to the dynamical system of spatial shifts on the attractor. Thus, the construction of embedding (5.22) clarifies the nature of the spatial chaotisity on the attractor of (2.1), in particular, it explains why we should use the factor $\left(\ln \frac{1}{\varepsilon}\right)^{-1}$ for the proper definition of the topological entropy $\widehat{h}_{s p}$ of the spatial dynamics on the attractor (see, e.g. [43] for a more detailed study of the phenomena of spatial chaos).

Thus, embedding (5.22) shows that the spatial dynamics on the attractor $\mathcal{A}$ is extremely chaotic. In particular, this embedding allows to realize (up to a homeomorphism) every finite dimensional dynamics by restricting the group of shitfts $\left\{T_{h}, h \in \mathbb{R}^{n}\right\}$ to the appropriate spatially invariant subset of $\mathcal{A}$. To be more precise, the following result holds.

Corollary 5.3. Let the assumptions of Theorem 5.1 hold, let $K \subset \mathbb{R}^{N}$ be an arbitray compact set in $\mathbb{R}^{N}, N \in \mathbb{N}$, and let $F_{1}, \cdots, F_{n}: K \rightarrow K$ be arbitrary pairwise commutative homeomorphisms, i.e.

$$
F_{i} \circ F_{j}=F_{j} \circ F_{i}, \quad i, j \in\{1, \cdots, n\} .
$$


Then, there exist a positive number $\gamma=\gamma(N)>0$ and a homeomorphism

$$
\widehat{\tau}: K \rightarrow \widehat{\tau}(K) \subset \mathcal{A}
$$

such that

$$
T_{\gamma l} \widehat{\tau}(k)=\widehat{\tau}\left(F_{1}^{l_{1}} \circ \cdots \circ F_{n}^{l_{n}}(k)\right), \quad k \in K, \quad l \in \mathbb{Z}^{n},
$$

where $F_{i}^{l_{i}}$ denotes the $l_{i}$-th iteration of the map $F_{i}$.

Proof. Indeed, due to Theorem 5.3, it is sufficient to construct only the embedding $\bar{\tau}: K \rightarrow \mathcal{M}$ which satisfies (5.26). Moroever, without loss of generality, we may assume that $K \subset \mathbb{D}^{M^{n}}$, for the appropriate $M \in \mathbb{N}$, and $\left\{(k)_{m_{1}, \cdots, m_{n}} \in \mathbb{D}, \quad m_{i}=\right.$ $0,1, \cdots, M-1\}$ is the corresponding coordinate system in it. We define the map $\bar{\tau}: K \rightarrow \mathcal{M}$ by the following formula:

$\bar{\tau}(k)(l):=\left(F_{1}^{k_{1}} \circ \cdots \circ F_{n}^{k_{n}}(k)\right)_{r_{1}, \cdots, r_{n}}, l_{i}=k_{i} M+r_{i}, k_{i} \in \mathbb{Z}, r_{i} \in\{0, \cdots, M-1\}$

Then, obviously, this map satisfies (5.26) with $\gamma=M$ and the continuity of this embedding follows immediately from the fact that $F_{i}$ are homeomorphisms. Corollary 5.3 is proved.

In order to study the spatial complexity of an individual point at the attractor, it is natural to introduce (following [43]) the following quantity.

Definition 5.3. Let $u_{0} \in \mathcal{A}$. Then, by definition, the (modified) spatial entropy of $u_{0}$ is the following number:

$$
\widehat{h}_{s p}\left(u_{0}\right):=\widehat{h}_{s p}\left(H\left(u_{0}\right)\right), \text { where } H\left(u_{0}\right):=\left[T_{h} u_{0}, h \in \mathbb{R}^{n}\right]_{L_{l o c}^{\infty}\left(R^{n}\right)},
$$

where $[\cdot]_{V}$ denotes the closure in the space $V$.

Corollary 5.4. Let the assumptions of Theorem 5.1 hold. Then, for every point $u_{0} \in \mathcal{A}$, its spatial entropy is finite. Moreover, there exist points $u_{0} \in \mathcal{A}$ the spatial entropy of which is strictly positive:

$$
0<\widehat{h}_{s p}\left(u_{0}\right)<\infty
$$

Indeed, the first assertion of the corollary follows immediately from Theorem 4.2 and the second one follows from Theorem 5.1 and from the obvious fact that the dynamical system $\left\{\mathcal{M}, \mathcal{T}_{l}\right\}$ is topologically transitive (i.e. possesses dense orbits).

Remark 5.3. It is proved in [43] that, under some natural assumptions on (2.1), spatial entropy (5.27) preserves under the temporal evolution:

$$
\widehat{h}_{s p}\left(S_{t} u_{0}\right)=\widehat{h}_{s p}\left(u_{0}\right), \quad u_{0} \in \mathcal{A} \text {. }
$$


$\S 6$ The spatial Dynamical system in the EXTended Phase spaCe.

Our next task is to extend Theorem 5.3 to the case of temporal dynamics. To this end, we construct the auxiliary dynamical system for which the direction $t$ will be 'spatial' and, applying the algorithm of studying the spatial chaos given in Section 5 to that system, we obtain the description of the temporal chaos for initial system (2.1).

As in Sections 4 and 5, we consider only spatially homogeneous case

$$
\Omega=\mathbb{R}^{n}, \quad g \equiv 0, \quad L(x):=L \in \mathbb{R}^{n}, \quad f(0)=0 .
$$

Moreover, without loss of generality, we may assume that the vector $L$ has the form

$$
L:=L e_{1}, \quad e_{1}:=(1,0, \cdots, 0), \quad L \in \mathbb{R}_{+}
$$

(the general case may be reduced to this one by the appropriate spatial rotation).

We now introduce the following auxiliary parabolic boundary value problem in the half-space $x=\left(x_{1}, x^{\prime}\right) \in \mathbb{R}_{+} \times \mathbb{R}^{n-1}$ :

$$
\left\{\begin{array}{l}
\partial_{t} u=a\left(\partial_{x_{1}}^{2} u+\Delta_{x^{\prime}} u\right)-L \partial_{x_{1}} u-\lambda_{0} u-f(u), \quad t \in \mathbb{R}, \quad x^{\prime} \in \mathbb{R}^{n-1}, \\
\left.u\right|_{x_{1}=0}=u^{0}, \quad x_{1} \geq 0
\end{array}\right.
$$

We consider this boundary problem as a (formal) evolution equation with respect to the variable $x_{1}$. In order to indicate this idea in a more clear way, we introduce new variables

$$
\eta:=x_{1}, \quad y=\left(y_{1}, \cdots, y_{n}\right)=\left(y_{1}, y^{\prime}\right):=\left(t, x^{\prime}\right) .
$$

In these variables problem (6.3) has the following view:

$$
\left\{\begin{array}{l}
a\left(\partial_{\eta}^{2} u+\Delta_{y^{\prime}} u\right)-L \partial_{\eta} u-\lambda_{0} u-f(u)=\partial_{y_{1}} u, \quad y \in \mathbb{R}^{n}, \quad \eta \geq 0, \\
\left.u\right|_{\eta=0}=u^{0} .
\end{array}\right.
$$

The following theorem shows that problem (6.5) defines indeed a dynamical system with respect to $\eta$, if $L$ is large enough.

Theorem 6.1. Let the assumptions of Theorem 2.1 hold and (6.2) be satisfied. We also assume that there exists a nonnegative constant $\Lambda_{0} \geq 0$ such that

$$
L \Lambda_{0}-\left(a_{+}-2 a_{-}\left(a_{+}\right)^{-1} a_{-}\right) \Lambda_{0}^{2}-K>0,
$$

where $a_{+}:=1 / 2\left(a+a^{*}\right), a_{-}:=1 / 2\left(a-a^{*}\right)$ and $K$ is the same as in (2.3). Then, for every $u^{0} \in \Psi_{b}\left(\mathbb{R} \times \mathbb{R}^{n-1}\right):=\left\{u^{0}, \partial_{t} u^{0} \in W_{b}^{(1-1 /(2 q), 2-1 / q), q}\left(\mathbb{R}^{n}\right)\right\}$, problem $(6.5)$ has a unique bounded solution $u(\eta, y)$, i.e. the unique solution in the class

$$
u, \partial_{t} u \in W_{b}^{(1,2), q}\left(\mathbb{R}_{t} \times\left(\mathbb{R}_{+, x_{1}} \times \mathbb{R}_{x^{\prime}}^{n-1}\right)\right) .
$$

Proof. The existence of a solution for problem (6.5) follows from Theorems 2.1 and 2.2 (even without assumption (6.6)). Indeed, it follows from these theorems that problem (6.5) with additional conditions $y_{1}>-N$ and $\left.u\right|_{y_{1}=-N}=u_{0}$ (where 
$u_{0} \in W_{b}^{2, q}\left(\mathbb{R}_{+} \times \mathbb{R}^{n}\right)$ such a function that $u_{0} \equiv 0$, if $\eta>1,\left\|u_{0}\right\|_{b, 2, q} \leq\left\|u^{0}\right\|_{\Psi_{b}}$, and the first compatibility condition at $\eta=0, y_{1}=-N$ is satisfied) has a solution $u_{N}$, for every $N \in \mathbb{N}$. Moreover, estimate (2.9) implies that

$$
\left\|u_{N}(t), B_{x_{0}}^{1}\right\|_{2, q}+\left\|\partial_{t} u_{N}(t), B_{x_{0}}^{1}\right\|_{0, q} \leq Q\left(\left\|u^{0}\right\|_{\Psi_{b}}\right)+Q\left(\|g\|_{L_{b}^{q}}\right),
$$

where the function $Q$ is independent of $N$ and $x_{0}$. Passing now to the limit $N \rightarrow \infty$ and using uniform estimate (6.8), we easily derive the existence of a solution for problem (6.6). We also note that estimate (2.9) implies the following estimate for this solution:

$$
\left\|u(t), B_{x_{0}}^{1}\right\|_{2, q}+\left\|\partial_{t} u(t), B_{x_{0}}^{1}\right\|_{0, q} \leq Q\left(\left\|u^{0}\right\|_{\Psi_{b}}\right) e^{-\alpha \eta}+Q\left(\|g\|_{L_{b}^{q}}\right),
$$

where $Q$ and $\alpha$ are independent of $t$ and $x_{0}$. We now recall that $\Psi_{b}\left(\mathbb{R}^{n}\right)$ is a trace space for space (6.7) to the hyperplane $x_{1}=0$ (see e.g. [28]), consequently, differentiating equation (6.5) with respect to $t \equiv y_{1}$ and applying the parabolic $L^{q}$-regularity theorem to the obtained equation, we derive from (6.9) that

$$
\begin{aligned}
\int_{T}^{T+1}\left(\left\|u(t), B_{x_{0}}^{1}\right\|_{2, q}^{q}+\left\|\partial_{t} u(t), B_{x_{0}}^{1}\right\|_{2, q}^{q}\right. & \left.+\left\|\partial_{t}^{2} u(t), B_{x_{0}}^{1}\right\|_{0, q}^{q}\right) d t \leq \\
& \leq Q\left(\left\|u^{0}\right\|_{\Psi_{b}}\right) e^{-\alpha x_{1}}+Q\left(\|g\|_{L_{b}^{q}}\right)
\end{aligned}
$$

where $Q$ and $\alpha$ are independent of $x_{0}$ and $T$. Thus, the existence of a solution of (6.5) in the class (6.7) is verified. Moreover, (due to the embedding theorem and the fact that $q>n+1)$, we have

$$
\|u\|_{C_{b}^{1}\left(\mathbb{R}_{+} \times \mathbb{R}^{n}\right)} \leq Q\left(\left\|u^{0}\right\|_{\Psi_{b}}\right)+Q\left(\|g\|_{L_{b}^{q}}\right)
$$

for the appropriate monotonic function $Q$.

Let us verify now the uniqueness of a solution under assumption (6.6). Indeed, let $u_{1}(\eta)$ and $u_{2}(\eta)$ be two solutions of problem (6.5) and let $v(\eta):=u_{1}(\eta)-u_{2}(\eta)$. Then, this function satisfies the following relation:

$$
\left\{\begin{array}{l}
a\left(\partial_{\eta}^{2} v+\Delta_{y^{\prime}} v\right)-L \partial_{\eta} v-\lambda_{0} v-l(\eta) v=\partial_{y_{1}} v, \quad y \in \mathbb{R}^{n}, \quad \eta \geq 0 \\
\left.v\right|_{\eta=0}=0
\end{array}\right.
$$

where $l(\eta):=\int_{0}^{1} f^{\prime}\left(s u_{1}(\eta)+(1-s) u_{2}(\eta)\right) d s$. Moroeover, due to (2.3) and (6.11), we have

$$
l(\eta) \geq-K \quad \text { and } \quad\|l\|_{C_{b}^{1}\left(\mathbb{R}_{+} \times \mathbb{R}^{n}\right)} \leq Q\left(\left\|\left.u_{i}\right|_{\eta=0}\right\| \|_{\Psi_{b}}\right) .
$$

Let us now introduce the variable $\theta(\eta):=e^{-\Lambda_{0} \eta} v(\eta)$, where $\Lambda_{0} \geq 0$ is the same as in (6.6). Then, we have the following equation:

$$
a\left(\partial_{\eta}^{2} \theta+\Delta_{y^{\prime}} \theta\right)-\left(L-2 a \Lambda_{0}\right) \partial_{\eta} \theta-\left(L \Lambda_{0}-a \Lambda_{0}^{2}-l(\eta)\right) \theta-\lambda_{0} \theta=\partial_{y_{1}} \theta
$$

Let us multiply equation (6.14) by $e^{-\varepsilon\left(\left|\eta-\eta_{0}\right|+\left|y-y_{0}\right|\right)} \theta(\eta):=\psi_{\varepsilon}(\eta, y) \theta$, where $\eta_{0} \geq 0$, $y_{0} \in \mathbb{R}^{n}$ and $\varepsilon$ is a small parameter which will be fixed below, and integrate over $(\eta, y) \in \mathbb{R}_{+} \times \mathbb{R}^{n}$ (since $v$ is bounded and $\Lambda_{0} \geq 0$ then $\theta$ is also bounded and, 
consequently, all integrals obtained below have a sense). Then, we derive, after the integration by parts, using inequality (2.11) and evident estimates, that

$$
\begin{gathered}
-\left\langle a_{+} \partial_{\eta} \theta . \partial_{\eta} \theta, \psi_{\varepsilon}\right\rangle-\left\langle a_{+} \nabla_{y^{\prime}} \theta \cdot \nabla_{y^{\prime}} \theta, \psi_{\varepsilon}\right\rangle+2 \Lambda_{0}\left|\left\langle a_{-} \partial_{\eta} \theta . \theta, \psi_{\varepsilon}\right\rangle\right|-\lambda_{0}\left\langle|\theta|^{2}, \psi_{\varepsilon}\right\rangle \\
-\left\langle\left(L \Lambda_{0}-a_{+} \Lambda_{0}^{2}-K\right) \theta . \theta, \psi_{\varepsilon}\right\rangle+C \varepsilon\left\langle\left|\partial_{\eta} \theta\right|^{2}+\left|\nabla_{y^{\prime}} \theta\right|^{2}+|\theta|^{2}, \psi_{\varepsilon}\right\rangle \geq 0,
\end{gathered}
$$

here and below $\langle u, v\rangle$ denotes the standard inner product in $L^{2}\left(\mathbb{R}_{+} \times \mathbb{R}^{n}\right)$. Estimating the third term in (6.15) as follows:

$$
2 \Lambda_{0}\left|\left\langle a_{-} \partial_{\eta} \theta . \theta, \psi_{\varepsilon}\right\rangle\right| \geq-1 / 2\left\langle a_{+} \partial_{\eta} \theta . \partial_{\eta} \theta, \psi_{\varepsilon}\right\rangle+2 \Lambda_{0}^{2}\left\langle a_{-}\left(a_{+}\right)^{-1} a_{-} \theta . \theta, \psi_{\varepsilon}\right\rangle
$$

we obtain the inequality

$$
\begin{array}{r}
1 / 2\left\langle a_{+} \partial_{\eta} \theta . \partial_{\eta} \theta, \psi_{\varepsilon}\right\rangle+\left\langle a_{+} \nabla_{y^{\prime}} \theta . \nabla_{y^{\prime}} \theta, \psi_{\varepsilon}\right\rangle+\lambda_{0}\left\langle|\theta|^{2}, \psi_{\varepsilon}\right\rangle+ \\
\left\langle\left(L \Lambda_{0}-\left(a_{+}-2 a_{-}\left(a_{+}\right)^{-1} a_{-}\right) \Lambda_{0}^{2}-K\right) \theta \cdot \theta, \psi_{\varepsilon}\right\rangle \leq \\
\leq C \varepsilon\left\langle\left|\partial_{\eta} \theta\right|^{2}+\left|\nabla_{y^{\prime}} \theta\right|^{2}+|\theta|^{2}, \psi_{\varepsilon}\right\rangle .
\end{array}
$$

Due to condition (6.6), the third term in (6.16) is nonnegative, consequently, there exists a positive constant $\mu>0$ (independent of $\varepsilon, \eta_{0}$ and $y_{0}$ ) such that

$$
(\mu-C \varepsilon)\left\langle\left|\partial_{\eta} \theta\right|^{2}+\left|\nabla_{y^{\prime}} \theta\right|^{2}+|\theta|^{2}, \psi_{\varepsilon}\right\rangle \leq 0,
$$

which implies that $\theta \equiv 0$ if $\varepsilon$ is small enough and finishes the proof of Theorem 6.1.

Corollary 6.1. Let the assumptions of Theorem 6.1 hold. Then, problem (6.5) defines a semigroup $\left\{\mathcal{S}_{\eta}, \eta \geq 0\right\}$ in the phase space $\Psi_{b}\left(\mathbb{R}^{n}\right)$ :

$$
\mathcal{S}_{\eta}: \Psi_{b} \rightarrow \Psi_{b}, \quad \eta \geq 0, \quad \mathcal{S}_{\eta} u^{0}:=u(\eta)
$$

where $u(\eta)$ is a solution of $(6.5)$, with $u(0)=u^{0}$. Moreover, this semigroup possesses the following estimate:

$$
\left\|\mathcal{S}_{\eta} u^{0}\right\|_{\Psi_{b}} \leq Q\left(\left\|u^{0}\right\|_{\Psi_{b}}\right) e^{-\alpha \eta}+Q\left(\|g\|_{L_{b}^{q}}\right)
$$

for the appropriate positive $\alpha>0$ and monotoinic function $Q$.

Indeed, estimate (6.19) is an immediate corollary of (6.10) and the definition of the trace space $\Psi_{b}$. The existence of the semigroup follows then from the main assertion of Theorem 6.1.

Our task now is to study dynamical system (6.18). At the first step, we show that this system is Lipschitz continuous in the spaces $\Psi_{\phi_{\varepsilon, y_{0}}}$, where $\phi_{\varepsilon, y_{0}}(y):=e^{-\varepsilon\left|y-y_{0}\right|}$ (see Definitions 1.2 and 1.5), if $\varepsilon>0$ is small enough.

Corollary 6.2. Let the assumptions of Theorem 6.1 hold and let $u_{1}(\eta)$ and $u_{2}(\eta)$ be two bounded solutions of (6.5), with different 'initial values'. Then, the following estimate is valid:

$$
\left\|u_{1}(\eta)-u_{2}(\eta)\right\|_{\Psi_{\phi_{\varepsilon}, y_{0}}} \leq C e^{\left(\Lambda_{0}-\varepsilon\right) \eta}\left\|u_{1}(0)-u_{2}(0)\right\|_{\Psi_{\phi_{\varepsilon}, y_{0}}},
$$


where $\varepsilon>0$ is small enough and the constant $C$ depends only on $\varepsilon$ and $\left\|u_{i}(0)\right\|_{\Phi_{b}}$, $i=1,2$ (but is independent of $y_{0} \in \mathbb{R}^{n}$ ).

Proof. Let, as in the proof of Theorem $6.1, \theta(\eta):=e^{-\Lambda_{0} \eta}\left(u_{1}(\eta)-u_{2}(\eta)\right)$. Then, this function satisfies equation (6.14), with non-zero boundary conditions $\left.\theta\right|_{\eta=0}=$ $u_{1}(0)-u_{2}(0)$. Let us now introduce a function $w(\eta, y):=\bar{w}(t, x)$ (the extention of the function $u_{1}(0)-u_{2}(0)$ inside of the domain $\left.\mathbb{R}_{+} \times \mathbb{R}^{n}\right)$ which belongs to class (6.7) and satisfies the following condition:

$$
\bar{w}(t, x) \equiv 0, \text { for } x_{1} \geq 1
$$

and the following inequality:

$$
\begin{aligned}
&\|\bar{w}\|_{W^{(1,2), q}\left([T, T+1] \times\left(B_{x_{0}}^{1} \cap \Omega_{+}\right)\right)}+\left\|\partial_{t} \bar{w}\right\|_{W^{(1,2), q}\left([T, T+1] \times\left(B_{x_{0}}^{1} \cap \Omega_{+}\right)\right)} \leq \\
& \leq C\left\|u_{1}(0)-u_{2}(0)\right\|_{\Psi\left([T-1, T+2] \times\left(B_{x_{0}}^{2} \cap \partial \Omega_{+}\right)\right)},
\end{aligned}
$$

where $\Omega_{+}:=\mathbb{R}_{+, x_{1}} \times \mathbb{R}_{x^{\prime}}^{n-1}$, the constant $C$ is independent of $T \in \mathbb{R}$ and $x_{0} \in \Omega_{+}$ and the space $\Psi$ is defined in (2.5). Such an extension exists since $\Psi_{b}$ is the trace space for functions of class $(6.7)$. Let $\theta_{1}(\eta):=\theta(\eta)-w(\eta)$. Then, this function, obviously, satisfies the non-homogeneous analogue of (6.14):

$$
\begin{array}{r}
a\left(\partial_{\eta}^{2} \theta_{1}+\Delta_{y^{\prime}} \theta_{1}\right)-\left(L-2 a \Lambda_{0}\right) \partial_{\eta} \theta_{1}-\left(L \Lambda_{0}-a \Lambda_{0}^{2}-l(\eta)\right) \\
\theta_{1}-\lambda_{0} \theta_{1}= \\
=\partial_{y_{1}} \theta_{1}+h(\eta)
\end{array}
$$

where $\left.\theta_{1}\right|_{\eta=0}=0$ and, according to (6.13), (6.21) and (6.22), the function $h(\eta):=$ $h(\eta, y)=\bar{h}(t, x)$ satisfies

$$
\begin{aligned}
& \|\bar{h}\|_{L^{q}\left([T, T+1] \times\left(B_{x_{0}}^{1} \cap \Omega_{+}\right)\right)}\left\|\partial_{t} \bar{h}\right\|_{L^{q}\left([T, T+1] \times\left(B_{x_{0}}^{1} \cap \Omega_{+}\right)\right)} \leq \\
& \leq C_{1}\left\|u_{1}(0)-u_{2}(0)\right\|_{\Psi\left([T-1, T+2] \times\left(B_{x_{0}}^{2} \cap \partial \Omega_{+}\right)\right)}
\end{aligned}
$$

where the constant $C_{1}$ depends on $\left\|u_{i}(0)\right\|_{\Psi_{b}}$, but is independent of $T \in \mathbb{R}$ and $x_{0}:=\left(\eta, x_{0}^{\prime}\right) \in \Omega$. We also note that the right-hand side of (6.24) vanishes if $\eta \geq 2$, since, in this case, $B_{x_{0}}^{2} \cap \partial \Omega_{+}=\varnothing$.

Multiplying now equation (6.23) by $e^{-\varepsilon\left|\eta-\eta_{0}\right|-\varepsilon\left|y-y_{0}\right|} \theta_{1}$ and arguing as in the proof of Theorem 6.1 , we derive that, for sufficiently small $\varepsilon>0$ the following estimate holds:

$$
\begin{aligned}
\left\langle\left|\partial_{\eta} \theta_{1}\right|^{2}+\left|\nabla_{y^{\prime}} \theta_{1}\right|^{2}+\left|\theta_{1}\right|^{2}, e^{-\varepsilon\left|\eta-\eta_{0}\right|-\varepsilon\left|y-y_{0}\right|}\right\rangle & \leq \\
& \leq C^{\prime \prime}\left\langle|h|^{2}, e^{-\varepsilon\left|\eta-\eta_{0}\right|-\varepsilon\left|y-y_{0}\right|}\right\rangle,
\end{aligned}
$$

where the constant $C^{\prime \prime}$ is independent of $\eta_{0}$ and $y_{0}$. Estimates (6.24) and (6.25) imply that

$$
\begin{aligned}
& \left\|\theta_{1}\right\|_{L^{2}\left([T, T+1] \times\left(B_{x_{0}}^{1} \cap \Omega_{+}\right)\right)} \leq \\
& \quad \leq C_{2} e^{-\varepsilon \eta}\left\|u_{1}(0)-u_{2}(0)\right\|_{\Psi_{\phi_{\varepsilon, y_{0}}}}, \quad y_{0}:=\left(T, x_{0}^{\prime}\right), \quad x_{0}:=\left(\eta, x_{0}^{\prime}\right),
\end{aligned}
$$

where $C_{2}$ depends on $\left\|u_{i}(0)\right\|_{\Psi_{b}}$, but is independent of $T \in \mathbb{R}$ and $x_{0} \in \Omega_{+}$. 
We now recall that, due to the standard interior regularity theory applied to parabolic equation (6.23) (see e.g. [28]), we have the following estimate:

$$
\begin{gathered}
\left\|\theta_{1}\right\|_{W^{(1,2), q}\left([T, T+1] \times\left(B_{x_{0}}^{1} \cap \Omega_{+}\right)\right)}+\left\|\partial_{t} \theta_{1}\right\|_{W^{(1,2), q}\left([T, T+1] \times\left(B_{x_{0}}^{1} \cap \Omega_{+}\right)\right)} \leq \\
\leq C_{3}\left(\left\|\theta_{1}\right\|_{L^{2}\left([T-1, T+1] \times\left(B_{x_{0}}^{2} \cap \Omega_{+}\right)\right)}+\|h\|_{L^{q}\left([T-1, T+1] \times\left(B_{x_{0}}^{2} \cap \Omega_{+}\right)\right)}+\right. \\
\left.+\left\|\partial_{t} h\right\|_{L^{q}\left([T-1, T+1] \times\left(B_{x_{0}}^{2} \cap \Omega_{+}\right)\right)}\right),
\end{gathered}
$$

where $C_{3}$ is independent of $T \in \mathbb{R}$ and $x_{0} \in \Omega_{+}$. Inserting estimates (6.26), (6.24) into the right-hand side of (6.27) and using the definition of the trace space $\Psi$, we obtain, after simple calculations, that

$$
\left\|\theta_{1}(\eta)\right\|_{\Psi\left(B_{y_{0}}^{1}\right)} \leq C_{4} e^{-\varepsilon \eta}\left\|u_{1}(0)-u_{2}(0)\right\|_{\Psi_{\phi_{\varepsilon}, y_{0}}\left(\mathbb{R}^{n}\right)}
$$

and, consequently

$$
\left\|u_{1}(\eta)-u_{2}(\eta)\right\|_{\Psi\left(B_{y_{0}}^{1}\right)} \leq C_{5} e^{\left(\Lambda_{0}-\varepsilon\right) \eta}\left\|u_{1}(0)-u_{2}(0)\right\|_{\Psi_{\phi_{\varepsilon}, y_{0}}\left(\mathbb{R}^{n}\right)},
$$

where the constants $C_{4}$ and $C_{5}$ depend on $\left\|u_{i}(0)\right\|_{\Psi_{b}}$ and $\varepsilon$, but are independent of $y_{0} \in \mathbb{R}^{n}$. Multiplying this estimate by $e^{-\varepsilon_{1}\left|y_{0}-z\right|}, \varepsilon_{1}<\varepsilon$, integrating over $y_{0} \in \mathbb{R}^{n}$ and using estimate (1.7), we derive (6.20). Corollary 6.2 is proved.

Corollary 6.3. Let the assumptions of Theorem 6.1 hold. Then, the semigroup $\left\{\mathcal{S}_{\eta}, \eta>0\right\}$ defined by expression (6.18) possesses a locally compact attractor $\mathcal{A}_{s p}$ (i.e. $\left(\Psi_{b}, \Psi_{l o c}\right)$-attractor, see Definition 3.1). Moreover, this attractor has the following structure:

$$
\mathcal{A}_{s p}=\left.\mathcal{K}\right|_{x_{1}=0}
$$

where $\mathcal{K}$ is the same as in Theorem 3.1.

Proof. Indeed, according to the attractors existence theorem for abstract semigroups (see e.g. [4], [24] or [34]), it is sufficient to verify the following conditions:

1. The semigroup (6.18) is $\Psi_{l o c}$-continuous on every $\Psi_{b}$-bounded set.

2. There exists an absorbing set $B \subset \Psi_{b}$ for this semigroup, which is compact in $\Psi_{l o c}$-topology.

We note that the first condition is an immediate corollary of estimate (6.20). Let us verify the second one. It follows from estimate (6.19) that the set

$$
B_{R}:=\left\{u^{0}:\left\|u^{0}\right\|_{\Psi_{b}} \leq R\right\}
$$

is an absorbing set for semigroup (6.18), if $R$ is large enough (which is not compact in $\left.\Psi_{l o c}\right)$. We claim, however, that the set $B:=\mathcal{S}_{1} B_{R}$ is a desired absorbing set which is (pre)compact in $\Psi_{l o c}$.

Indeed, it follows from (6.10) and from the embedding theorem $(q>n+1)$ that

$$
\|f(u)\|_{C_{b}^{1}\left(\mathbb{R}_{+} \times \mathbb{R}^{n}\right)} \leq C=C(R),
$$

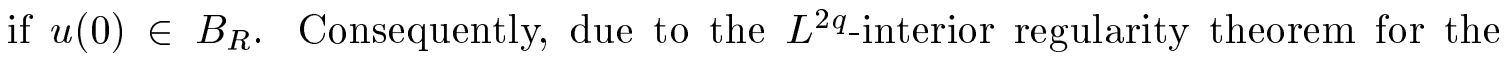
parabolic equations (see (6.27), where $q$ is replaced by $2 q$ ) and due to the explicit description of the corresponding trace space at $\eta=0$, we have

$$
\|u(1)\|_{W_{b}^{(1-1 /(4 q), 2-1 /(2 q)}\left(\mathbb{R}^{n}\right)}+\left\|\partial_{y_{1}} u(1)\right\|_{W_{b}^{(1-1 /(4 q), 2-1 /(2 q)}\left(\mathbb{R}^{n}\right)} \leq Q_{1}(R),
$$


if $u(0)=u^{0} \in B_{R}$. There now remains to note that the space in the left-hand side of $(6.31)$ is compactly embedded to $\Psi_{l o c}$.

Thus, all the conditions of the attractors existence theorem are verified for semigroup (6.18) and, consequently, it possesses a locally compact attractor $\mathcal{A}_{s p}$. Description (6.29) also follows from this abstract theorem and from the evident fact that the sets of all bounded trajectories for semigroups (6.18) and (2.41) coinside. Corollary 6.3 is proved.

Our next task is to verify that the semigroup $\left\{\mathcal{S}_{\eta}, \eta>0\right\}$ is differentiable with respect to the initial data $u^{0}$. To this end, we need the solvability result for the corresponding equation of variations.

Corollary 6.4. Let the assumptions of Theorem 6.1 hold, $u(\eta):=\mathcal{S}_{\eta} u^{0}, u^{0} \in \Psi_{b}$, be an arbitrary solution of (6.5), and a function $h(\eta, y)$ be such that

$$
e^{-\Lambda_{0} \eta} h(\eta, y), e^{-\Lambda_{0} \eta} \partial_{y_{1}} h(\eta, y) \in L_{b}^{q}\left(\mathbb{R}_{+} \times \mathbb{R}^{n}\right)
$$

Then, for every $w^{0} \in \Psi_{b}$, the following problem:

$$
\left\{\begin{array}{l}
a\left(\partial_{\eta}^{2} w+\Delta_{y^{\prime}} w\right)-L \partial_{\eta} w-\lambda_{0} w-f^{\prime}(u(\eta)) w=\partial_{y_{1}} w+h \\
\left.w\right|_{\eta=0}=w^{0}
\end{array}\right.
$$

has a unique solution in the class

$$
w \in L_{e^{-\Lambda_{0} \eta}}^{\infty}\left(\mathbb{R}_{+}, \Psi_{b}\left(\mathbb{R}^{n}\right)\right)
$$

and the following estimate is valid, for a sufficiently small $\varepsilon>0$ and for every $y_{0} \in \mathbb{R}^{n}$ :

$$
\begin{aligned}
& \|w(\eta)\|_{\Psi_{\phi_{\varepsilon}, y_{0}}}^{q} \leq C e^{q\left(\Lambda_{0}-\varepsilon\right) \eta}\left\|w^{0}\right\|_{\Psi_{\phi_{\varepsilon}, y_{0}}}^{q}+ \\
& +C e^{q \Lambda_{0} \eta} \int_{0}^{\infty} e^{-q \varepsilon\left|\eta-\eta_{0}\right|-q \Lambda_{0} \eta_{0}}\left(\left\|h\left(\eta_{0}\right)\right\|_{L_{\phi_{\varepsilon}, y_{0}}^{q}}^{q}+\left\|\partial_{y_{1}} h\left(\eta_{0}\right)\right\|_{L_{\phi_{\varepsilon}, y_{0}}^{q}}^{q}\right) d \eta_{0}
\end{aligned}
$$

where the constant $C$ depends on $\left\|u^{0}\right\|_{\Psi_{b}}$ and $\varepsilon$, but is independent of $y_{0}$.

The proof of this Corollary is completely analogous to that of Theorem 6.1 and Corollary 6.2, so we omit it here.

Remark 6.1. It is essential that we consider only such solutions $w$ of (6.33) which grow as $\eta \rightarrow+\infty$ not faster than $e^{\Lambda_{0} \eta}$. Usually, there exists a number of other solutions of (6.33) which grow faster than $e^{\Lambda_{0} \eta}$, but all these solutions are out of the consideration, due to assumption (6.34).

Theorem 6.2. Let the assumptions of Theorem 6.1 hold and let $u(\eta)$ and $u_{1}(\eta)$ be two arbitrary solutions of (6.5). We define the function $w(\eta)$ as a unique solution (in class (6.34)) of problem (6.33), with $h \equiv 0$ and $w(0)=u(0)-u_{1}(0)$. Then, the following estimate is valid, for sufficiently small $\varepsilon>0, \mu>0$, and for every $y_{0} \in \mathbb{R}^{n}$ :

$$
\left\|u(\eta)-u_{1}(\eta)-w(\eta)\right\|_{\Psi_{\phi_{\varepsilon}, y_{0}}} \leq C e^{\Lambda_{0} \eta}\left\|u(0)-u_{1}(0)\right\|_{\Psi_{b}}^{\mu}\left\|u(0)-u_{1}(0)\right\|_{\Psi_{\phi_{\varepsilon}, x_{0}}}
$$


where the constant $C$ depends on $\|u(0)\|_{\Psi_{b}},\left\|u_{1}(0)\right\|_{\Psi_{b}}, \varepsilon$, and $\mu$, but is independent of $y_{0} \in \mathbb{R}^{n}$.

Proof. We set $v(\eta):=u(\eta)-u_{1}(\eta)$ and $\theta(t):=v(t)-w(t)$. Then, the last function satisfies the following equation:

$$
\left\{\begin{array}{l}
a\left(\partial_{\eta}^{2} \theta+\Delta_{y^{\prime}} \theta\right)-L \partial_{\eta} \theta-\lambda_{0} \theta-f^{\prime}(u(\eta)) \theta=\partial_{y_{1}} \theta+h(\eta) \\
\left.\theta\right|_{\eta=0}=0
\end{array}\right.
$$

where

$$
h(\eta):=\int_{0}^{1}\left[f^{\prime}(u(\eta)-s v(\eta))-f^{\prime}(u(\eta))\right] d s v(\eta)
$$

Applying estimate (6.35) to equation (6.37), we have

$$
\begin{aligned}
& \quad\|\theta(\eta)\|_{\Psi_{\phi_{\varepsilon}, y_{0}}}^{q} \leq \\
& \leq C e^{q \Lambda_{0} \eta} \int_{0}^{\infty} e^{-q \varepsilon\left|\eta-\eta_{0}\right|-q \Lambda_{0} \eta_{0}}\left(\|h(\eta)\|_{L_{\phi_{\varepsilon}, y_{0}}^{q}\left(\mathbb{R}^{n}\right)}^{q}+\left\|\partial_{y_{1}} h(\eta)\right\|_{L_{\phi_{\varepsilon}, y_{0}}^{q}\left(\mathbb{R}^{n}\right)}^{q}\right) d \eta_{0} .
\end{aligned}
$$

Thus, there remains to obtain the appropriate estimates for function (6.38) and its $y_{1}$-derivative. To this end, we recall that $f^{\prime} \in C^{1}$ and, consequently

$$
\left|f^{\prime}\left(\xi_{1}\right)-f^{\prime}\left(\xi_{2}\right)\right| \leq Q_{\mu}\left(\left|\xi_{1}\right|+\left|\xi_{2}\right|\right)\left|\xi_{1}-\xi_{2}\right|^{\mu}
$$

for every $\xi_{i} \in \mathbb{R}^{k}$ and every $0 \leq \mu \leq 1$ (here $Q_{\mu}$ is a monotonic function depending on $\mu$ and $f$. Therefore, (due to the embedding $\Psi_{b} \subset C$ and Corollary 6.1)

$$
\|h(\eta)\|_{L_{\phi_{\varepsilon}, y_{0}}^{q}\left(\mathbb{R}^{n}\right)} \leq C\|v(\eta)\|_{\Psi_{b}}^{\mu}\|v(\eta)\|_{\Psi_{\phi_{\varepsilon}, y_{0}}}
$$

where $C$ depends on $\mu,\|u(0)\|_{\Psi_{b}}$ and $\left\|u_{1}(0)\right\|_{\Psi_{b}}$, but is independent of $y_{0}$. Estimating the right-hand side of $(6.41)$ by $(6.20)$, we have

$$
\|h(\eta)\|_{L_{\phi_{\varepsilon}, y_{0}}^{q}\left(\mathbb{R}^{n}\right)} \leq C_{1} e^{(1+\mu)\left(\Lambda_{0}-\varepsilon\right) \eta}\|v(0)\|_{\Psi_{b}}^{\mu}\|v(0)\|_{\Psi_{\phi_{\varepsilon}, y_{0}}} .
$$

Arguing analogously, but using the fact that $f^{\prime \prime} \in C^{1}$ and $C^{1} \subset \Psi$, we have

$$
\left\|\partial_{y_{1}} h(\eta)\right\|_{L_{\phi_{\varepsilon, y_{0}}}^{q}\left(\mathbb{R}^{n}\right)} \leq C_{2} e^{(1+\mu)\left(\Lambda_{0}-\varepsilon\right) \eta}\|v(0)\|_{\Psi_{b}}^{\mu}\|v(0)\|_{\Psi_{\phi_{\varepsilon}, y_{0}}} .
$$

Seting $\mu>0$ in such way that $(1+\mu)\left(\Lambda_{0}-\varepsilon\right) \leq \Lambda_{0}$ and inserting estimates (6.42) and (6.43) into the right-hand side of (6.39), we obtain estimate (6.36) after simple calculations. Theorem 6.2 is proved.

Corollary 6.5. Let the assumptions of Theorem 6.1 hold. Then, semigroup (6.18) is Frechet differntiable in $\Psi_{b}$, for every fixed $\eta \geq 0$ and its Frechet derivative $D_{u^{0}} \mathcal{S}_{\eta}\left(u^{0}\right) \xi:=w_{\xi}(\eta)$, where $\xi \in \Psi_{b}$ and $w_{\xi}(\eta)$ is a unique solution of (6.33), with $h \equiv 0$ and $w(0)=\xi$. Moreover,

$$
\mathcal{S}_{\eta} \in C^{1+\mu}\left(\Psi_{b}, \Psi_{b}\right)
$$


and the following estimates hold, for every $u_{1}^{0}, u_{2}^{0} \in \Psi_{b}$ :

$$
\begin{gathered}
\left\|\mathcal{S}_{\eta}\left(u_{1}^{0}\right)-\mathcal{S}_{\eta}\left(u_{2}^{0}\right)-D_{u^{0}} \mathcal{S}_{\eta}\left(u_{1}^{0}\right)\left(u_{1}^{0}-u_{2}^{0}\right)\right\|_{\Psi_{b}} \leq C e^{\Lambda_{0} \eta}\left\|u_{1}^{0}-u_{2}^{0}\right\|_{\Psi_{b}}^{1+\mu} \\
\left\|D_{u_{0}} \mathcal{S}_{\eta}\left(u_{1}^{0}\right)-D_{u_{0}} \mathcal{S}_{\eta}\left(u_{2}^{0}\right)\right\|_{\mathcal{L}\left(\Psi_{b}, \Psi_{b}\right)} \leq C e^{\Lambda_{0} \eta}\left\|u_{1}^{0}-u_{2}^{0}\right\|_{\Psi_{b}}^{\mu}
\end{gathered}
$$

where $\mu>0$ and the constant $C$ depends on $\left\|u_{i}^{0}\right\|_{\Psi_{b}}, i=1,2$.

Indeed, applying the supremum with respect to $y_{0} \in \mathbb{R}^{n}$ to the both sides of (6.36) and using (1.19), we obtain estimate (6.45). Estimate (6.46) is a standard corollary of (6.45).

Remark 6.2. In contrast to the case of semigroups, generated by the evolution equations, we cannot gurantee that $\mathcal{S}_{\eta} \in C^{2}$, even for the case where $f \in C^{\infty}$. In a fact, in order to obtain the regularity $C^{N}$, one should require, in addition, assumption (6.6) to be satisfied not only for $\Lambda=\Lambda_{0}$, but also for $\Lambda=2 \Lambda_{0}, \cdots, \Lambda=$ $N \Lambda_{0}$. Fortunately, regularity (6.44) is enough for what follows.

Remark 6.3. The method of introducing the spatial dynamical systems (and the so-called spatial reduction) was initiated in [26] in order to study the elliptic boundary problems in cylindrical domains from the dynamical point of view, see also [8], [36] and the references therein for the further developement of this method and its applications to elliptic boundary problems. Nevertheless, to the best of our knoledge, this method has never been directly aplied to study the spatial 'dynamics' generated by parabolic equations.

\section{$\S 7$ ThE SPATIAL DYNAMiCAL SYSTEM NEAR THE EXPONENTIALLY UNSTABLE EQUILIBRIUM.}

In this Section, we construct the infinite dymensional unstable manifold of zero equilibrium of the spatial dynamical system $\left\{\mathcal{S}_{\eta}, \eta \geq 0\right\}$ constructed in the previous Section. We recall that, due to Corollary 6.5, this semigroup is of the class $C^{1+\mu}$, for some positive $\mu>0$, and the linearized semigroup at $u^{0}=0$

$$
\mathcal{S}_{\eta}^{0}:=D_{u^{0}} \mathcal{S}_{\eta}(0)
$$

can be determined by the following expression: $\mathcal{S}_{\eta}^{0} v^{0}:=w(\eta)$, where the function $w(\eta)$ is a unique (due to Corollary 6.4) solution of the following problem:

$$
\left\{\begin{array}{l}
a\left(\partial_{\eta} w+\Delta_{y^{\prime}} w\right)-L \partial_{\eta} w-\lambda_{0} w-f^{\prime}(0) w=\partial_{y_{1}} w \\
\left.w\right|_{\eta=0}=v^{0}
\end{array}\right.
$$

which belongs to class (6.34). Moreover, estimate (6.35) allows to extend this semigroup in a unique way to the semigroup, acting in the weight space $\Psi_{\phi}$, for every weight function $\phi$ with sufficiently small exponential growth rate, in particular, for every $\phi$ with polynomial growth rate (see Definition 1.1).

The following proposition describes the spectrum of this linarized semigroup.

Proposition 7.1. Let the assumptions of Theorem 6.1 hold. Then, the spectrum of the operator $\mathcal{S}_{\eta}^{0}, \eta>0$, in $\Psi_{b}$ coinsides with its spectrum in $\Psi_{\phi}$, where $\phi$ is an 
arbitrary weight function with polynomial growth rate and can be found from the following expression:

$$
\begin{aligned}
\sigma\left(\mathcal{S}_{\eta}^{0}\right)=\{0\} \cup\left\{\lambda=e^{\beta \eta}\right. & : \exists \beta \in \mathbb{C}, \operatorname{Re} \beta \leq \Lambda_{0}, \exists \xi \in \mathbb{R}^{n}, \text { such that } \\
& \left.\operatorname{det}\left(a\left(\beta^{2}-\left|\xi^{\prime}\right|^{2}\right)-L \beta-\lambda_{0}-f^{\prime}(0)-i \xi_{1}\right)=0\right\} .
\end{aligned}
$$

The assertion of this Proposition is more or less standard and can be verified using the Fourier transform and the classical theorems on multiplicators in $L^{q}\left(\mathbb{R}^{n}\right)$ (see, e.g. [35]).

Our next task is to verify that linearized semigroup (7.1) is exponentially unstable, if (5.2) is fulfilled.

Proposition 7.2. Let the assumption of Theorem 6.1 hold and let, in addition, condition (5.2) is also fulfilled, i.e.

$$
\sigma\left(a\left(\partial_{\eta}^{2}+\Delta_{y^{\prime}}\right)-L \partial_{\eta}-\lambda_{0}-f^{\prime}(0)\right) \cap\{\operatorname{Re} \lambda>0\} \neq \varnothing .
$$

Then, linearized semigroup (7.1) is exponentially unstable, i.e

$$
\sigma\left(\mathcal{S}_{\eta}^{0}\right) \cap\{|\lambda|=\eta\} \neq \varnothing
$$

Proof. Indeed, it follows from (7.4) that there exist a point $\beta_{0} \in i \mathbb{R}$, a point $\xi^{\prime} \in$ $\mathbb{R}^{n-1}$ and a point $\lambda_{0}^{\prime}$, Re $\lambda_{0}^{\prime}>0$ such that

$$
\operatorname{det}\left(a\left(\beta_{0}^{2}-\left|\xi^{\prime}\right|^{2}\right)-L \beta_{0}-\lambda_{0}-f^{\prime}(0)-\lambda_{0}^{\prime}\right)=0
$$

On the other hand, condition (6.6) obviously implies that

$$
\operatorname{Re} \sigma\left(a\left(\Lambda_{0}^{2}-\left|\xi^{\prime}\right|^{2}\right)-L \Lambda_{0}-\lambda_{0}-f^{\prime}(0)\right)<0 .
$$

It now follows from (7.6), (7.7) and the continuity arguments that there exists a point $\beta \in \mathbb{C}, 0<\operatorname{Re} \beta<\Lambda_{0}$, such that

$$
\sigma\left(a\left(\beta^{2}-\left|\xi^{\prime}\right|^{2}\right)-L \beta_{0}-\lambda_{0}-f^{\prime}(0)\right) \cap\{i \mathbb{R}\} \neq \varnothing .
$$

Consequently, there exists $\xi_{1} \in \mathbb{R}$ such that

$$
\operatorname{det}\left(a\left(\beta^{2}-\left|\xi^{\prime}\right|^{2}\right)-L \beta-\lambda_{0}-f^{\prime}(0)-i \xi_{1}\right)=0 \text {. }
$$

The assertion of the proposition is an immediate corollary of (7.9) and (7.3).

We are now ready to formulate the main result of this Section which is the analogue of Theorem 5.1 for the spatial dynamical system.

Theorem 7.1. Let the assumptions of Theorem 6.1 hold and assumption (5.2) be satisfied. Then, for every $N>>1$, there exist a positive number $\sigma>0$, a vector $\xi_{0} \in \mathbb{R}^{n}, \sigma<\left|\xi_{0}\right|$, a positive number $r_{0}=r_{0}(N)>0$ and a commuting with the group $\left\{T_{h}^{y}, h \in \mathbb{R}^{n}\right\}$ of 'spatial' shifts map

$$
\mathcal{V}_{0}: B\left(r, 0, \mathbb{B}_{\xi_{0}, \sigma}\right) \rightarrow \underset{49}{\mathcal{A}_{s p},} \quad T_{h}^{y} \circ \mathcal{V}_{0}=\mathcal{V}_{0} \circ T_{h}^{y}
$$


such that, for every $y_{0} \in \mathbb{R}^{n}$, the following estimates hold:

$$
\left\{\begin{array}{l}
\left\|\mathcal{V}_{0}\left(u_{1}\right)-\mathcal{V}_{0}\left(u_{2}\right), B_{y_{0}}^{1}\right\|_{\Psi} \leq C_{N} \sup _{y \in \mathbb{R}^{n}} \frac{\left\|u_{1}-u_{2}, B_{y}^{1}\right\|_{0}, \infty}{\left(1+\left|y-y_{0}\right|^{2 N}\right)^{1 / 2}} \\
\left\|u_{1}-u_{2}, B_{y_{0}}^{1}\right\|_{0, \infty} \leq C_{N} \sup _{y \in \mathbb{R}^{n}} \frac{\left\|\mathcal{V}_{0}\left(u_{1}\right)-\mathcal{V}_{0}\left(u_{2}\right), B_{y}^{1}\right\|_{\Psi}}{\left(1+\left|y-y_{0}\right|^{2 N}\right)^{1 / 2}}
\end{array}\right.
$$

where the constant $C_{N}$ depends only on $N$ and independednt of $y_{0}$.

Moreover, there exist a vector $\vec{l} \in \mathbb{R}^{k}$ and a linear operator $\mathbb{S}_{0}: \mathbb{B}_{\xi_{0}, \sigma}\left(\mathbb{R}^{n}\right) \rightarrow$ $\Psi_{b}\left(\mathbb{R}^{n}\right)^{k}$ such that

$$
\mathbb{S}_{0}\left(u_{0}\right) . \vec{l} \equiv \operatorname{Re} u_{0}, \text { for every } u_{0} \in \mathbb{B}_{\xi_{0}, \sigma}\left(\mathbb{R}^{n}\right)
$$

and, for every $u_{0} \in B\left(r, 0, \mathbb{B}_{\xi_{0}, \sigma}\right)$, the following estimate holds:

$$
\left\|\mathcal{V}_{0}\left(u_{0}\right)-S_{0}\left(u_{0}\right)\right\|_{\Psi_{b}\left(\mathbb{R}^{n}\right)} \leq C\left\|u_{0}\right\|_{L_{b}^{\infty}\left(\mathbb{R}^{n}\right)}^{1+\mu},
$$

where $1 \geq \mu>0$ is the same as in (6.44).

Proof. Let $L:=\mathcal{S}_{1}, L_{0}:=\mathcal{S}_{1}^{0}$, and $P:=L-L_{0}$. Then, due to Corollary 6.5

$$
\left\|P v^{0}\right\|_{\Psi_{\phi_{\varepsilon}, y_{0}}} \leq C\left(\left\|v^{0}\right\|_{\Psi_{b}}\right)\left\|v^{0}\right\|_{\Psi_{b}}^{\mu}\left\|v^{0}\right\|_{\Psi_{\phi_{\varepsilon}, y_{0}}},
$$

where $C$ is independent of $y_{0}$.

According to description (6.29), it is sufficient construct a sufficiently large set of bounded backward solutions (i.e. defined for $\eta<0$ ) for problem (6.5) (which can be parametrized by the points from $\left.B\left(r, 0, \mathbb{B}_{\xi_{0}, \sigma}\right)\right)$. Instead of considering the continuous dynamics generated by (6.5), we consider the 'equivalent' discrete one

$$
v(m+1)=L_{0} v(m)+P v(m), \quad m \in \mathbb{Z}_{-},
$$

which acts on the space of sequences $\{v(m)\} \in L^{\infty}\left(\mathbb{Z}_{-}, \Psi_{b}\right)$. Our plan now is to solve (7.15) near zero equilibrium using the implicit function theorem. To this end, we first study the linear non-homogeneous analogue of (7.15)

$$
w(m+1)-L_{0} w(m)=h(m) .
$$

Lemma 7.1. Let the above assumptions hold. Then, for every $\alpha>r\left(L_{0}\right):=$ $\left|\sigma\left(L_{0}\right)\right|>1$ and for every $h \in L_{\alpha^{-m}}^{\infty}\left(\mathbb{Z}_{-}, \Psi_{b}\right)$, there exists a unique solution $w \in$ $L_{\alpha^{-m}}^{\infty}\left(\mathbb{Z}_{-}, \Psi_{b}\right)$ such that

$$
\|w\|_{L_{\alpha^{-m}}^{\infty}\left(\mathbb{Z}_{-}, \Psi_{b}\right)} \leq C\|h\|_{L_{\alpha^{-m}}^{\infty}\left(\mathbb{Z}_{-}, \Psi_{b}\right)}
$$

and, consequently, the linear operator

$$
\mathbb{T}_{\alpha}: L_{\alpha^{-m}}^{\infty}\left(\mathbb{Z}_{-}, \Psi_{b}\right) \rightarrow L_{\alpha^{-m}}^{\infty}\left(\mathbb{Z}_{-}, \Psi_{b}\right)
$$

is well defined by the expression $\left(\mathbb{T}_{\alpha} h\right)(l):=w(l)$. Moreover, for every $N \in \mathbb{N}$ and $y_{0} \in \mathbb{R}^{n}$

$$
\|w\|_{L_{\alpha^{-m}}^{\infty}\left(\mathbb{Z}_{-}, \Psi_{\varphi_{N}, y_{0}}\right)} \underset{50}{\left.C_{N}\|h\|_{L_{\alpha^{-m}}^{\infty}\left(\mathbb{Z}_{-}, \Psi_{\varphi_{N}, y_{0}}\right.}\right)}
$$


where the weight $\varphi_{N, y_{0}}$ is defined by (1.6) and the constant $C_{N}$ is independent of $y_{0} \in \mathbb{R}^{n}$.

Indeed, the operator $\mathbb{T}_{\alpha}$ is given by the following expression:

$$
\left(\mathbb{T}_{\alpha} h\right)(l):=\sum_{m=-\infty}^{l} L_{0}^{l-m} h(m) .
$$

The fact that definition (7.19) is correct, together with estimate (7.17), follows from the assumption $\alpha>\left|\sigma\left(L_{0}\right)\right|$ and from the standard formula for the spectral radius of $L_{0}$. The fact that the function $w:=\mathbb{T}_{\alpha} h$ satisfies (7.16) can be easily verified in a direct way. Estimate (7.18) can be obtained in the same way as (7.17) using, in addition, the fact that the spectra of $L_{0}$ in $\Psi_{b}$ and $\Psi_{\varphi_{N, y_{0}}}$ coinside (according to Proposition 7.1).

Let us consider now homogeneous problem $(7.16)(h \equiv 0)$.

Lemma 7.2. Let the above assumptions hold. Then, there exist a positive number $\alpha_{0}, r\left(L_{0}\right)>\alpha_{0}>1$, satisfying $\alpha_{0}^{1+\mu}>r\left(L_{0}\right)$, a vector $\xi_{0} \in \mathbb{R}^{n}$, a number $\sigma>0, \sigma<\left|\xi_{0}\right|$, a vector $\vec{l} \in \mathbb{R}^{k}$ and a linear operator

$$
\mathbb{S}: \mathbb{B}_{\xi_{0}, \sigma}\left(\mathbb{R}^{n}\right) \rightarrow L_{\alpha_{0}^{-m}}^{\infty}\left(\mathbb{Z}_{-}, \Psi_{b}\left(\mathbb{R}^{n}\right)\right)^{k}
$$

such that

1. $w(m):=\left(\mathbb{S} u_{0}\right)(m)$ is a solution of $(7.16)$ with $h \equiv 0$, for every $u_{0} \in \mathbb{B}_{\xi_{0}, \sigma}$.

2. $\left(\mathbb{S}_{0} u_{0}\right) . \vec{l} \equiv \operatorname{Re} u_{0}$, for every $u_{0} \in \mathbb{B}_{\xi_{0}, \sigma}$, where $\mathbb{S}_{0} u_{0}:=\left(\mathbb{S} u_{0}\right)(0)$.

3. For every $N \in \mathbb{N}$, every $y_{0} \in \mathbb{R}^{n}$ and every $u_{0} \in \mathbb{B}_{\xi_{0}, \sigma}$, the following estimate is valid:

$$
\left\|\mathbb{S} u_{0}\right\|_{L_{\alpha_{0}^{-m}}^{\infty}\left(\mathbb{Z}_{-}, \Psi_{\varphi_{q N}, y_{0}}\right)} \leq C_{N}\left\|u_{0}\right\|_{L_{\varphi_{N, y_{0}}}^{\infty}\left(\mathbb{R}^{n}\right)}
$$

where the constant $C_{N}$ is independent of $y_{0}$ and, consequently

$$
\left\|\mathbb{S} u_{0}\right\|_{L_{\alpha_{0}^{-m}}^{\infty}\left(\mathbb{Z}_{-}, \Psi_{b}\right)} \leq C\left\|u_{0}\right\|_{L^{\infty}\left(\mathbb{R}^{n}\right)}
$$

Proof. According to Proposition 7.2, there exist a point $\xi_{0} \in \mathbb{R}^{n}$ and a point $\beta_{0}=$ $\beta\left(\xi_{0}\right): 0<\operatorname{Re} \beta_{0}<\Lambda_{0}$ such that

$$
\operatorname{det}\left(a\left(\beta\left(\xi_{0}\right)^{2}-\left|\xi_{0}^{\prime}\right|^{2}\right)-L \beta\left(\xi_{0}\right)-\lambda_{0}-f^{\prime}(0)-i \xi_{0}^{1}\right)=0
$$

We note that equation (7.22) defines an algebraic ( $2 n$-sheeted) function $\widehat{\beta}(\xi)$. Consequently, without loss of generality, we may assume that $\left(\xi_{0}, \beta_{0}\left(\xi_{0}\right)\right)$ is not a branch point of this function. Moreover, without loss of generality, we may assume also that $\xi_{0} \neq 0$ and $(1+\mu) \operatorname{Re} \beta_{0}>\ln r\left(L_{0}\right)$, where $r\left(L_{0}\right)$ is a spectral radius of $L_{0}$ which, due to Proposition 7.1, can be calculated as follows:

$$
\ln r\left(L_{0}\right)=\max \left\{\operatorname{Re} \widehat{\beta}(\xi): \xi \in \mathbb{R}^{n}, \operatorname{Re} \widehat{\beta}(\xi) \leq \Lambda_{0}\right\}
$$


Thus, there exist a neighbourhood $B_{\xi_{0}}^{r^{\prime}}$ and smooth functions $\beta: B_{\xi_{0}}^{r^{\prime}} \rightarrow B_{\beta_{0}}^{r^{\prime \prime}}$ and $e: B_{\xi_{0}}^{r^{\prime}} \rightarrow \mathbb{C}^{k}$ such that $\beta\left(\xi_{0}\right)=\beta_{0}$ and

$$
\left(a\left(\beta(\xi)^{2}-\left|\xi^{\prime}\right|^{2}\right)-L \beta(\xi)-\lambda_{0}-f^{\prime}(0)-i \xi^{1}\right) e(\xi)=0, \text { for every } \xi \in B_{\xi_{0}}^{r^{\prime}}
$$

Moreover, we may assume (see e.g. [43]) that the eigenvalue $e(\xi)$ is normalized in such way that

$$
e(\xi) . \vec{l}=1, \quad \text { for every } \xi \in B_{\xi_{0}}^{r^{\prime}}
$$

for the appropriate constant vector $\vec{l} \in \mathbb{R}^{k}$.

We also assume that $r^{\prime}>0$ is small enough that $(1+\mu)\left(\operatorname{Re} \beta_{0}-r^{\prime \prime}\right)>\ln r\left(L_{0}\right)$ and define $\alpha_{0}:=e^{\operatorname{Re} \beta_{0}-r^{\prime \prime}}$. Moreover, we define the exponent $\sigma>0$ in such way that

$$
\operatorname{supp} \widehat{u}_{0} \subset B_{\xi_{0}}^{r^{\prime} / 2}, \text { for every } u_{0} \in \mathbb{B}_{\xi_{0}, \sigma}\left(\mathbb{R}^{n}\right) \text {, }
$$

where $\widehat{u}_{0}$ denotes the Fourier transform of the function $u_{0}$.

There now remains to define the operator $\mathbb{S}^{\prime}: \mathbb{B}_{\xi_{0}, \sigma} \rightarrow L_{\alpha_{0}^{-m}}^{\infty}\left(\mathbb{Z}_{-}, \Psi_{b}\left(\mathbb{R}^{n}, \mathbb{C}\right)\right)^{k}$ by the expression:

$$
\widehat{\mathbb{S}^{\prime}\left(\widehat{\left.u_{0}\right)(m)}\right.}(\xi):=e^{m \beta(\xi)} \widehat{u_{0}}(\xi) e(\xi), \quad u_{0} \in \mathbb{B}_{\xi_{0}, \sigma}
$$

and to define finally $\mathbb{S}\left(u_{0}\right):=\operatorname{Re} \mathbb{S}^{\prime}\left(u_{0}\right)$. Then, it is not difficult to verify (analogously to [43]) that the operator thus obtained satisfies all the assumptions of the lemma. Lemma 7.2 is proved.

Lemma 7.3. Let the above assumptions hold and let $\mathbb{S}_{0}$ be the same as in Lemma 7.2. Then, the following estimates hold:

$$
C_{N}^{-1}\left\|u_{0}\right\|_{L_{\varphi_{N}, y_{0}}^{\infty}\left(\mathbb{R}^{n}\right)} \leq\left\|\mathbb{S}_{0} u_{0}\right\|_{L_{\varphi_{N}, y_{0}}^{\infty}\left(\mathbb{R}^{n}\right)} \leq C_{N}\left\|u_{0}\right\|_{L_{\varphi_{N, y_{0}}}^{\infty}\left(\mathbb{R}^{n}\right)},
$$

where the constant $C_{N}$ is independent of $y_{0}$.

Indeed, the right inequality of (7.28) is an immediate corollary of Lemma 7.2 and the proof of the left one is based on the formula $\mathbb{S}_{0} u_{0}=\operatorname{Re} u_{0}$ and on the fact that every function from $\mathbb{B}_{\xi_{0}, \sigma}$, with $\sigma<\left|\xi_{0}\right|$, is determined in a unique way by its real part (see [43] for the details).

Let us verify some important properties of the operator $P$ acting on the space of sequences.

Lemma 7.4. Let the above assumptions hold. Then, the operator $\mathcal{P}$, determined by the expression

$$
(\mathcal{P} v)(m):=P v(m)
$$

is of the class

$$
\mathcal{P} \in C^{1+\mu}\left(L_{\alpha^{-m}}^{\infty}\left(\mathbb{Z}_{-}, \Psi_{b}\right), L_{\alpha^{-m(1+\mu)}}^{\infty}\left(\mathbb{Z}_{-}, \Psi_{b}\right)\right),
$$


for every $\alpha>1$ and the following estimate holds, for every $N$ and $y_{0}$ :

$$
\left.\|\mathcal{P} v\|_{L_{\alpha^{-m}(1+\mu)}^{\infty}\left(\mathbb{Z}_{-}, \Psi_{\varphi_{N}, y_{0}}\right)} \leq C_{N}\|v\|_{L_{\alpha^{-m}}^{\infty}\left(\mathbb{Z}_{-}, \Psi_{b}\right)}^{\mu}\|v\|_{L_{\alpha^{-m}}^{\infty}\left(\mathbb{Z}_{-}, \Psi_{\varphi_{N}, y_{0}}\right.}\right),
$$

where the constant $C_{N}$ is independent of $y_{0} \in \mathbb{R}^{n}$.

Proof. Estimate (7.31) is an immediate corollary of (7.14) and (1.7). Let us now verify the differentiability. Let $v_{1}, v_{2} \in L_{\alpha^{-m}}^{\infty}\left(\mathbb{Z}_{-}, \Psi_{b}\right)$. Then, due to Corollary 6.5

$$
\begin{aligned}
& (7.32) \quad\left\|P\left(v_{1}(m)\right)-P\left(v_{2}(m)\right)-D_{u_{0}} P\left(v_{1}(m)\right)\left(v_{1}(m)-v_{2}(m)\right)\right\|_{\Psi_{b}}= \\
& \left\|L\left(v_{1}(m)\right)-L\left(v_{2}(m)\right)-D_{u_{0}} L\left(v_{1}(m)\right)\left(v_{1}(m)-v_{2}(m)\right)\right\|_{\Psi_{b}} \leq C\left\|v_{1}(m)-v_{2}(m)\right\|_{\Psi_{b}}^{1+\mu} .
\end{aligned}
$$

Multiplying (7.32) by $\alpha^{-(1+\mu) m}$ and taking the supremum over $m \in \mathbb{Z}_{-}$, we obtain that the map $\mathcal{P}$ is differentiable (and verify the evident formula for its derivative). There remains to verify that the derivative is Hölder continuous. Let, in addition, $\xi \in L_{\alpha^{-m}}^{\infty}\left(\mathbb{Z}_{-}, \Psi_{b}\right)$ be an arbitrary sequence. Then, due to (6.46)

$$
\begin{aligned}
& \left\|\left[D_{u_{0}} P\left(v_{1}(m)\right)-D_{u_{0}} P\left(v_{2}(m)\right)\right] \xi(m)\right\|_{\Psi_{b}}= \\
& \quad=\left\|\left[D_{u_{0}} L\left(v_{1}(m)\right)-D_{u_{0}} L\left(v_{2}(m)\right)\right] \xi(m)\right\|_{\Psi_{b}} \leq C\left\|v_{1}-v_{2}\right\|_{\Psi_{b}}^{\mu}\|\xi(m)\|_{\Psi_{b}} .
\end{aligned}
$$

Multiplying now this relation by $\alpha^{-(1+\mu) m}$ and taking the supremum over $m \in \mathbb{Z}_{-}$, we verify that the derivative is indeed Hölder continuous and finish the proof of Lemma 7.4.

We are now ready to complete the proof of the theorem. To this end, we rewrite equation (7.15) as follows:

$$
v=\mathbb{S} u_{0}+\mathbb{T}_{\alpha_{0}^{1+\mu}} \mathcal{P} v
$$

where $\alpha_{0}>1$ is the same as in Lemma $7.2, u_{0} \in \mathbb{B}_{\xi_{0}, \sigma}$ and $v \in L_{\alpha_{0}^{-m}}^{\infty}\left(\mathbb{Z}_{-}, \Psi_{b}\right)$ and solve it near 0 using the implicit function theorem. Indeed, let us define the map

$$
\mathcal{F}: L_{\alpha_{0}^{-m}}^{\infty}\left(\mathbb{Z}_{-}, \Psi_{b}\right) \times \mathbb{B}_{\xi_{0}, \sigma}\left(\mathbb{R}^{n}\right) \rightarrow L_{\alpha_{0}^{-m}}^{\infty}\left(\mathbb{Z}_{-}, \Psi_{b}\right)
$$

by the following expression

$$
\mathcal{F}\left(v, u_{0}\right):=v-\mathbb{S} u_{0}-\mathbb{T}_{\alpha_{0}^{1+\mu}} \mathcal{P} v
$$

According to our construction, $\alpha_{0}^{1+\mu}>r\left(L_{0}\right)$ and, consequently (due to Lemmata 7.1 and 7.4), function (7.36) is well defined. Moreover, it follows from Lemma 7.4 that this function is of the class $C^{1+\mu}$ and $D_{v} \mathcal{F}(0,0)=\mathrm{Id}$. Thus, due to the implicit function theorem, there exist $r_{0}>0$ and a $C^{1}$-map $\mathcal{V}: B\left(r_{0}, 0, \mathbb{B}_{\xi_{0}, \sigma}\right) \rightarrow$ $L_{\alpha_{0}^{-m}}^{\infty}\left(\mathbb{Z}_{-}, \Psi_{b}\right)$ such that

$$
\mathcal{F}\left(\mathcal{V}\left(u_{0}\right), u_{0}\right)=0
$$

and, consequently, the function $v:=\mathcal{V}\left(u_{0}\right)$ solves $(7.15)$. We now set $\mathcal{V}_{0}\left(u_{0}\right):=$ $\mathcal{V}\left(u_{0}\right)(0)$. We claim that this map satisfies all the assertions of Theorem 7.1. Indeed, since $D_{u_{0}} \mathcal{V}(0)=0$, then

$$
\left\|\mathcal{V}\left(u_{0}\right)\right\|_{L_{\alpha_{0}^{-m}}^{\infty}\left(\mathbb{Z}_{-}, \Psi_{b}\right)} \leq C\left\|u_{0}\right\|_{L^{\infty}} \leq C r_{0}
$$


It follows from (7.31), (7.37), and (7.38) that

$$
\left\|\mathcal{V}\left(u_{0}\right)-\mathbb{S} u_{0}\right\|_{L_{\alpha_{0}^{-m}}^{\infty}}\left(\mathbb{Z}_{-}, \Psi_{b}\right) \leq C\left\|u_{0}\right\|_{L^{\infty}}^{1+\mu}
$$

Restricting (7.39) to $m=0$, we obtain (7.13). Let us verify estimates (7.11). Let $u_{1}, u_{2} \in B\left(r_{0}, 0, \mathbb{B}_{\xi_{0}, \sigma}\right)$. Then, it follows from (7.37), (7.17), and from (6.45) that

$$
\begin{aligned}
\| \mathcal{V}\left(u_{1}\right)- & \mathcal{V}\left(u_{2}\right)-\mathbb{S}\left(u_{1}-u_{2}\right) \|_{L_{\alpha_{0}^{-m}}^{\infty}\left(\mathbb{Z}_{-}, \Psi_{\varphi_{N}, y_{0}}\right)} \leq \\
& \left.\leq C_{N} \sum_{i=1}^{2}\left\|\mathcal{V}\left(u_{i}\right)\right\|_{L_{\alpha_{0}^{-m}}^{\infty}\left(\mathbb{Z}_{-}, \Psi_{b}\right)}^{\mu}\left\|\mathcal{V}\left(u_{1}\right)-\mathcal{V}\left(u_{2}\right)\right\|_{L_{\alpha_{0}^{-m}}^{\infty}\left(\mathbb{Z}_{-}, \Psi_{\varphi_{N}, y_{0}}\right.}\right)
\end{aligned}
$$

Using now estimates (7.38), (7.20) and decreasing $r_{0}>0$, if necerssary, we derive from (7.40) that

$$
\left\|\mathcal{V}\left(u_{1}\right)-\mathcal{V}\left(u_{2}\right)\right\|_{L_{\alpha_{0}^{-m}}^{\infty}\left(\mathbb{Z}_{-}, \Psi_{\varphi_{q N}, y_{0}}\right)} \leq C_{N}\left\|u_{1}-u_{2}\right\|_{L_{\varphi_{N}, y_{0}}^{\infty}}
$$

for all $u_{1}, u_{2} \in B\left(r_{0}(N), 0, \mathbb{B}_{\xi_{0}, \sigma}\right)$ and for sufficiently small positive $r_{0}(N)>0$. The first estimate of (7.13) is an immediate corollary of this estimate (with $N$ replaced by $N-n-1)$. Let us now verify the second one. To this end, we derive from $(7.40)$ and from (7.28) that

$$
\begin{aligned}
C_{N}^{-1} \| u_{1}- & u_{2}\left\|_{L_{\varphi_{N, x_{0}}}^{\infty}} \leq\right\| \mathcal{V}_{0}\left(u_{1}\right)-\mathcal{V}_{0}\left(u_{2}\right) \|_{L_{\varphi_{N}, x_{0}}^{\infty}}+ \\
& \left.+C_{N}^{\prime} \sum_{i=1}^{2}\left\|\mathcal{V}\left(u_{i}\right)\right\|_{L_{\alpha_{0}^{-m}}^{\infty}\left(\mathbb{Z}_{-}, \Psi_{b}\right)}^{\mu}\left\|\mathcal{V}\left(u_{1}\right)-\mathcal{V}\left(u_{2}\right)\right\|_{L_{\alpha_{0}^{-m}}^{\infty}\left(\mathbb{Z}_{-}, \Psi_{\varphi_{N, y_{0}}}\right.}\right)
\end{aligned}
$$

Inserting estimates (7.38) and (7.41) into the right-hand side of (7.42), we have

$$
\left\|u_{1}-u_{2}\right\|_{L_{\varphi_{N}, x_{0}}^{\infty}} \leq C\left\|\mathcal{V}_{0}\left(u_{1}\right)-\mathcal{V}_{0}\left(u_{2}\right)\right\|_{L_{\varphi_{N, x_{0}}}^{\infty}}+C_{N}^{\prime \prime} r_{0}^{\mu}\left\|u_{1}-u_{2}\right\|_{L_{\varphi_{N}, x_{0}}^{\infty}}
$$

Setting now $r_{0}=r_{0}(N)$ small enough, we obtain from (7.43) that

$$
\left\|u_{1}-u_{2}\right\|_{L_{\varphi_{N, x_{0}}}^{\infty}} \leq C_{1}\left\|\mathcal{V}_{0}\left(u_{1}\right)-\mathcal{V}_{0}\left(u_{2}\right)\right\|_{L_{\varphi_{N, x_{0}}}^{\infty}},
$$

for every $u_{1}, u_{2} \in B\left(r_{0}(N), 0, \mathbb{B}_{\xi_{0}, \sigma}\right)$. Thus, the second estimate of (7.13) is also verified. We also note that the fact that $\mathcal{V}_{0}$ commutes with 'spatial' shifts follows from the fact that all operators involving in equation (7.34) commute with these shifts and from the uniqueness part of the implicit function theorem.

Thus, there remains to verify embedding (7.10). Let $u_{0} \in B\left(r_{0}, 0, \mathbb{B}_{\xi_{0}, \sigma}\right)$. Then

$$
v(m):=\left\{\begin{array}{lll}
\mathcal{V}\left(u_{0}\right)(m) & \text { if } & m \in \mathbb{Z}_{-}, \\
L^{m}\left(u_{0}\right) & \text { if } & m \in \mathbb{N}
\end{array}\right.
$$

is a complete bounded trajectory of the discrete semigroup generated by the operator $L$. Moreover, it is also follows from Theorem 6.1 that the function $v(\eta):=$ $\mathcal{S}_{\nu} v(m)$, where $\eta=\nu+m, m \in \mathbb{Z}$ and $0 \leq \nu<1$, is a complete bounded trajectory 
of the continuous semigroup $\mathcal{S}_{\eta}$. Therefore, due to $(6.29), v(0)=\mathcal{V}\left(u_{0}\right) \in \mathcal{A}_{s p}$ and Theorem 7.1 is proved.

Remark 7.1. We have proved a little more: namely, that there exists a Liptschitz continuous embedding:

$$
\tilde{\mathcal{V}}:\left(B\left(r_{0}, 0, \mathbb{B}_{\xi_{0}, \sigma}\right), T_{h}^{y}\right) \rightarrow\left(\mathcal{K}, T_{h}^{y}\right),
$$

such that

$$
\begin{aligned}
C^{-1}\left\|u_{1}-u_{2}\right\|_{L_{\varphi_{N}, y_{0}}^{\infty}} \leq \\
\quad \leq\left\|\tilde{\mathcal{V}}\left(u_{1}\right)-\tilde{\mathcal{V}}\left(u_{2}\right)\right\|_{C_{e^{-\Lambda_{0}|\eta|}}\left(\mathbb{R}, \Phi_{\varphi_{q N}, y_{0}}\right)} \leq C\left\|u_{1}-u_{2}\right\|_{L_{\varphi_{N}, y_{0}}^{\infty}},
\end{aligned}
$$

for the appropriate constant $C$ which is independent of $y_{0}$. Indeed, this embedding is given by formula

$$
\tilde{\mathcal{V}}\left(u_{0}\right)(\eta):= \begin{cases}\mathcal{S}_{\nu} \mathcal{V}(m), \quad \nu=\{\eta\}, \quad m=[\eta] & \text { if } \eta<0 \\ \mathcal{S}_{\eta} \mathcal{V}_{0}\left(u_{0}\right) & \text { if } \eta>0\end{cases}
$$

Corollary 7.1. Let the assumptions of Theorem 7.1 hold. Then, for $\varepsilon<\varepsilon_{0}<1$, the follwowing estimate holds:

$$
\mathbb{H}_{\varepsilon}\left(\mathcal{A}_{s p}, L^{\infty}\left(y \in B_{0}^{R}\right)\right) \geq C R^{n} \ln \frac{1}{\varepsilon}
$$

Moreover, for $R=1$ and for every $\delta>0$, there exists $C_{\delta}>0$ such that

$$
\mathbb{H}_{\varepsilon}\left(\mathcal{A}_{s p}, L^{\infty}\left(B_{0}^{1}\right)\right) \geq C_{\delta}\left(\ln \frac{1}{\varepsilon}\right)^{n+1-\delta}
$$

The proof of estimates (7.48) and (7.49) is completely analogous to that given in Theorem 5.2 (only instead of (5.8) one should use (7.13)).

Corollary 7.2. Let the above assumptions hold. Then, the 'spatial' topological entropy (i.e. the entropy, which corresponds to the 'spatial' shifts $T_{h}^{y}$ ) is strictly positive:

$$
\widehat{h}^{\prime} s^{\prime}\left(\mathcal{A}_{s p}\right)>0
$$

(see Section 4).

Indeed, (7.50) is an immediate corollary of (7.48).

\section{$\S 8$ COMPlEXity OF TEMPORAL DYNAMICS IN RDS IN UNBOUNDED DOMAINS AND TEMPORAL CHAOS.}

In this Section, we return to study the dynamical system $\left\{S_{t}, t \geq 0\right\}$ and formulate several statements which are corollaries of the results obtained in Section 7 for the case of spatial dynamical system $\left\{\mathcal{S}_{\eta}, \eta \geq 0\right\}$. We start our consideration with the proof that the temporal (modified) topological entropy for the semigroup $S_{t}$ generated by equation (2.1) may be strictly positive. 
Theorem 8.1. Assume that equation (2.1) has form (6.3), the assumptions of Theorem 2.1 are satisfied and conditions (6.6) and (5.2) are fulfilled. Then, the (modified) topolgical entropy which corresponds to $V_{n}:=\operatorname{span}\left\{t, x_{2}, \cdots, x_{n}\right\}$ (see Definition 4.2) is strictly positive for the attractor $\mathcal{A}$ of this equation:

$$
\widehat{h}_{n}^{V_{n}}(\mathcal{A})>0 .
$$

Proof. According to Corollary 7.2

$$
\widehat{h}_{n}^{V_{n}}\left(\mathcal{A}_{s p}\right) \equiv \widehat{h}^{\prime} s^{\prime}\left(\mathcal{A}_{s p}\right)>0 .
$$

Then, analogously to Remark 4.5 (see also (7.46)), we have

$$
\widehat{h}_{n}^{V_{n}}(\mathcal{K})>0 .
$$

(Here we have implicitly used the fact that the sets of all bounded solutions for $S_{t}$ and $\mathcal{S}_{\eta}$ coinsides). Estimate (8.1) is an immediate corollary of (8.3) and (4.40) and Theorem 8.1 is proved.

Corollary 8.1. Let the assumptions of Theorem 8.1 hold. Then, the temporal (modified) topological entropy of the attractor $\mathcal{A}$ is strictly positive:

$$
\widehat{h}_{t}(\mathcal{A}):=\limsup _{\varepsilon \rightarrow 0}\left(\ln \frac{1}{\varepsilon}\right)^{-n} \lim _{T \rightarrow+\infty} \frac{1}{T} \mathbb{H}_{\varepsilon}\left(\mathcal{K}, L_{e^{-|x|}}^{\infty}\left([0, T] \times \mathbb{R}_{x}^{n}\right)\right)>0 .
$$

Indeed, (8.4) is an immediate corollary of (8.1) and Theorem 4.3.

Remark 8.1. Estimate (8.4) shows, in particular, that the classical topological entropy of the semigroup $S_{t}$ on $\mathcal{A}$ (which is defined analogously to (8.4), but without the factor $\left(\ln \frac{1}{\varepsilon}\right)^{-n}$ ) is infinite.

We also note that Theorem 8.1 hold not only for the transport term $\left(\vec{L}, \nabla_{x}\right) \equiv$ $L \partial_{x_{1}}$, but for all vectors $\vec{L}$ the norm $|\vec{L}|$ of which is large enough. Indeed, up to the appropriate rotation, the general transport term is equivalent to $|\vec{L}| \partial_{x_{1}}$.

Let us obtain now the analogue of Theorems 5.2 and 5.3 for the case of temporal dynamics. To this end, we need the following proposition.

Proposition 8.1. Let the assumptions of Theorem 4.1 hold and let, in addition, the diffusion matrix a satisfy

$$
a a^{*}=a^{*} a .
$$

Then, the map $\Pi_{0}: \mathcal{K} \rightarrow \mathcal{A}, \Pi_{0} u:=u(0)$ realizes a homeomorphism

$$
\Pi_{0}: \mathcal{K} \rightarrow \mathcal{A}
$$

where the sets $\mathcal{K}$ and $\mathcal{A}$ are endowed by the local topology of the spaces $C_{\text {loc }}\left(\mathbb{R}^{n+1}\right)$ and $C_{\text {loc }}\left(\mathbb{R}^{n}\right)$ respectively.

Proof. Indeed, since the sets $\mathcal{K}$ and $\mathcal{A}$ are compact and $\Pi_{0}(\mathcal{K})=\mathcal{A}$, then it is sufficient to verify that map (8.6) is injective. In other words, it is sufficient to verify that equation (2.1) possesses the property of backward uniqueness on the attractor. This fact is verified in [43] based on the results of [3], under the additional technical assumption (8.5) (to be more precise, it is verified in [43], for the case $L=0$, but the genaral case $L \neq 0$ is completely analogous). Proposition 8.1 is proved. 
Theorem 8.2. Let the assumptions of Theorem 8.1 and Proposition 8.1 hold. Then, there exists a homeomorphic embedding:

$$
\mathbb{V}: B\left(r_{0}, 0, \mathbb{B}_{\xi_{0}, \sigma}\right) \rightarrow \mathcal{A}
$$

where $\sigma, \xi_{0}$ and $r_{0}$ are the same as in Theorem $\% .1$ (for a some fixed $N \gg 1$ ) and all topologies are 'local' $\left(C_{\text {loc }}\left(\mathbb{R}^{n}\right)\right)$, such that

$$
S_{t} \mathbb{V}\left(u_{0}\right)=\mathbb{V}\left(T_{t}^{x_{1}} u_{0}\right), \quad T_{h}^{x_{i}} \mathbb{V}\left(u_{0}\right)=\mathbb{V}\left(T_{h}^{x_{i}} u_{0}\right), i=2, \cdots, n,
$$

for every $u_{0} \in B\left(r_{0}, 0, \mathbb{B}_{\xi_{0}, \sigma}\right), t \geq 0$ and $h \in \mathbb{R}$ (here and below $T_{h}^{x_{i}}:=T_{h e_{i}}$ is a spatial shift along the $\left.x_{i}\right)$. Moreover,

$$
\widehat{h}_{n}^{V_{n}}\left(\mathbb{V}\left(B\left(r_{0}, 0, \mathbb{B}_{\xi_{0}, \sigma}\right)\right)>0 .\right.
$$

Proof. Indeed, according to estimate (7.46), we have a homeomorphic embedding $\tilde{\mathcal{V}}$ of the set $B\left(r_{0}, 0, \mathbb{B}_{\xi_{0}, \sigma}\right)$ to $\mathcal{K}$ endowed by the topology of $L_{\text {loc }}^{\infty}\left(\mathbb{R}_{+}, \Psi_{\text {loc }}\left(\mathbb{R}^{n}\right)\right)$. But it is not difficult to show, using the standard interior estimates and the fact that $\mathcal{K}$ is bounded in $L^{\infty}\left(\mathbb{R}^{n}\right)$, that the topologies, endowed on $\mathcal{K}$ by the embeddings to $C_{l o c}\left(\mathbb{R}^{n+1}\right)$ and to $C_{l o c}\left(\mathbb{R}, \Psi_{l o c}\left(\mathbb{R}_{y}^{n}\right)\right)$, coinside. Consequently, $(7.45)$ is a homeomorphic embedding of $B\left(r_{0}, 0, \mathbb{B}_{\xi_{0}, \sigma}\right)$ to the space $\mathcal{K}$ endowed by the topology of $C_{l o c}\left(\mathbb{R}^{n+1}\right)$ as well.

We now define map (8.7) by the following expression:

$$
\mathbb{V}:=\Pi_{0} \circ \tilde{\mathcal{V}}
$$

where $\Pi_{0}$ is defined in Proposition 8.1. It is not difficult to verify that the map thus obtained satisies all the assertions of Theorem 8.2.

Combining Theorem 8.2 and Proposition 5.1, we obtain the following result (which is analogous to Theorem 5.3).

Theorem 8.3. Let the assumptions of Theorem 8.2 hold. Then, there exist a number $\alpha>0$ and a homeomorphic embedding

$$
\widehat{\tau}: \mathcal{M} \rightarrow \mathcal{A}
$$

such that

$$
S_{\alpha l} \widehat{\tau}\left(v_{0}\right)=\widehat{\tau}\left(\mathcal{T}_{l}^{x_{1}} v_{0}\right), \quad T_{\alpha l}^{x_{i}} \widehat{\tau}\left(v_{0}\right)=\widehat{\tau}\left(\mathcal{T}_{l}^{x_{i}} v_{0}\right), i=2, \cdots, n,
$$

for every $l \in \mathbb{Z}$ and every $v_{0} \in \mathcal{M}$. Moreover,

$$
\widehat{h}_{n}^{V_{n}}(\widehat{\tau}(\mathcal{M}))>0,
$$

where $V_{n}:=\operatorname{span}\left\{t, x_{2}, \cdots, x_{n}\right\}$.

Indeed, it is sufficient to take $\widehat{\tau}:=\mathbb{V} \circ \kappa$, where $\kappa$ is defined in Proposition 5.1.

Thus, embedding (8.10) shows that the spatio-temporal dynamics on the attractor $\mathcal{A}$ is also may be extremely chaotic. In particular, this embedding allows to realize (up to a homeomorphism) every finite dymensional dynamics by restricting the (semi)group $S_{t}$ to the appropriate spatially invariant subset of $\mathcal{A}$. To be more precies, the following result holds (compare with Corollary 5.3). 
Corollary 8.2. Let the assumptions of Theorem 8.2 hold, $K \subset \mathbb{R}^{N}$ be an arbitray compact set in $\mathbb{R}^{N}, N \in \mathbb{N}$, and $F_{1}, \cdots, F_{n}: K \rightarrow K$ be arbitrary pairwise commutative homeomorphisms, i.e.

$$
F_{i} \circ F_{j}=F_{j} \circ F_{i}, \quad i, j \in\{1, \cdots, n\} .
$$

Then, there exist a positive number $\gamma=\gamma(N)>0$ and a homeomorphism

$$
\tilde{\tau}: K \rightarrow \tilde{\tau}(K) \subset \mathcal{A}
$$

such that

$$
S_{\gamma l_{1}} \circ T_{\gamma l_{2}}^{x_{2}} \circ \cdots \circ T_{\gamma l_{n}}^{x_{n}} \tilde{\tau}(k)=\tilde{\tau}\left(F_{1}^{l_{1}} \circ \cdots \circ F_{n}^{l_{n}} k\right), \quad k \in K, \quad l \in \mathbb{Z}^{n},
$$

where $F_{i}^{l_{i}}$ denotes the $l_{i}$-th iteration of the map $F_{i}$.

The proof of this corollary is completely analogous to that of Corollary 5.3, so we omit it here.

In order to study the temporal complexity of individual point on the attractor, it is natural to introduce (analogously to Definition 5.3) the following quantity.

Definition 8.1. Let $u_{0} \in \mathcal{A}$. Then, by definition, the (modified) temporal entropy of $u_{0}$ is the following number:

$$
\widehat{h}_{t}\left(u_{0}\right):=\widehat{h}_{t}\left(\mathcal{H}_{t}\left(u_{0}\right)\right), \text { where } \mathcal{H}_{t}\left(u_{0}\right):=\left[S_{t} u_{0}, t \in \mathbb{R}_{+}\right]_{L_{\text {loc }}^{\infty}\left(R^{n}\right)},
$$

where $[\cdot]_{V}$ denotes the closure in the space $V$.

Corollary 8.3. Let the assumptions of Theorem 8.2 hold. Then, for every point $u_{0} \in \mathcal{A}$ its (modified) temporal entropy is finite. Moreover, there exist points $u_{0} \in \mathcal{A}$ the temporal entropy of which is strictly positive:

$$
0<\widehat{h}_{t}\left(u_{0}\right)<\infty .
$$

Indeed, the first assertion of the corollary follows immediately from Theorem 4.2 and the second one follows from Theorem 8.2 and from the evident fact that the dynamical system $\left\{\mathcal{T}_{l}^{x_{1}}, \mathcal{M}\right\}, l \in \mathbb{N}$, is topologically transitive (i.e. possesses dense orbits).

Let us consider, in conclusion, the simplest example of equation of type (2.1), for which our theory works.

Example 8.1. Let $\Omega=\mathbb{R}^{n}$. We consider the following analogue of the scalar $k=1$ Chafee-Infante equation with the transport term:

$$
\partial_{t} u=\Delta_{x} u-L \partial_{x_{1}} u+u-u^{3}, \quad x \in \mathbb{R}^{n} .
$$

Then, as it is not difficult to verify, the assumptions of Theorem 8.2 are satisfied, if $L>2$ and $n<6$. Thus, we have an exetremely chaotic temporal dynamics on the attractor (Theorems 8.1-8.3 and Corollaries 8.1-8.3 hold for equation (8.17), if $L>2$ ). In particular, there exists a great (uncountable) number of different time periodic solutions of this equation parametrized by the corresponding periodic trajectories of $\left(\mathcal{T}_{l}^{x_{1}}, \mathcal{M}\right), l \in \mathbb{Z}$.

We also note that the above results are not true without the transport term $L \partial_{x} u(!)$. Indeed, for $L=0$ (8.17) generates the so called extended gradient system and, consequently (see [22]), does not possess any time periodic solutions (at least in case $n<3$ ). This example gives a good illustration for the influence of the transport terms on the reaction-diffusion dynamics. 


\section{REFERENCES}

1. F.Abergel, Existence and Finite Dimensionality of the Global Attractor for Evolution Equations on Unbounded Domains, J. Diff. Equ. 83 (1990), 85-108.

2. V.Afromovich, A.Babin, and S.Chow, Spatial Chaotic Structure of Attractors of ReactionDiffusion Systems, Trans. Amer. Math. Soc 348 (1996), no. 12, 5031-5063.

3. S.Agmon, L.Nirenberg, Lower Bounds and Uniqueness Theorems for Solutions of Differential Equations in a Hilbert Space, Comm. Pure Appl. Math. 20 (1967), 207-229.

4. A.Babin, M.Vishik, Attractors of Evolutionary Equations, North Holland, Amsterdam, 1992.

5. A.Babin and M.Vishik, Attractors of Partial Differential Evolution Equations in an Unbounded Domain, Proc. Roy. Soc. Edinburgh Sect. A 116 (1990), no. 3-4, 221-243.

6. A.Babin, On Space-Chaotic Solutions to Scalar Parabolic Equations with Modulated Nonlinearities, Rus. Jour. Math. Phys. 3(3) (1995), 389-392.

7. A.Babin and B.Nicolaenko, Exponential Attractors of Reaction-Diffusion Systems in an Unbounded Domain, J. Dyn. Diff. Equ. 7 (4) (1995), 567-590.

8. À.Calsina, X.Mora, J.Solà-Morales, The Dynamical Approach to Elliptic Problems in Cylindrical Domains, and a Study of Their Parabolic Singular Limit, J. Diff. Eqns. 102 (1993), 244-304.

9. V.Chepyzhov and M.Vishik, Kolmogorov's $\varepsilon$-entropy for the Attractor of Reaction-Diffusion Equation, Math. Sbornik 189(2) (1998), 81-110.

10. F.Clement, X.Heimans, A.Angenent, et al., One-Parameter Semigroups, Moscow, 1992.

11. P.Collet, J.Eckmann, Extensive Properties of the Complex Ginzburg-Landau equation, Communication in Mathematical Physics 200 (1999), 699-722.

12. P.Collet and J.Eckmann, The Definition and Measurement of the Topological Entropy per Unit Volume in Parabolic PDE., Nonlinearity 12 (1999), 451-473.

13. P.Collet and J.Eckmann, Topological Entropy and E-entropy for Damped Hyperbolic Equations, Ann. Inst. Henri Poincaré 1 (2000), no. 4, 715-752.

14. V.Coti Zelati, M.Nolasco, Multibump Solutions for Hamiltonian Systems with Fast and Slow Forcing, Bollettino U.M.I. 8 2-B (1999), 585-608.

15. J.-P.Eckmann and J.Rougemont, Coarsening by Ginzburg-Landau Dynamics, Comm. Math. Phys. 199 (1999), 441-470.

16. M.Efendiev and A.Miranville, Finite Dimensional Attractors for RDE in $R^{n}$ with a Strong Nonlinearity, Disc. Cont. Dyn. Systems 15 (2) (1999), 399-424.

17. M.Efendiev, and S.Zelik, The Attractor for a Nonlinear Reaction-Diffusion System in an Unbounded Domain, Comm. Pure Appl. Math. 54 (2001), no. 6, 625-688.

18. M.Efendiev and S.Zelik, Attractors of the Reaction-Diffusion Systems with Rapidly Oscillating Coefficients and their Homojenization, DANSE FU-Berlin, Preprint 26/00 (2000), to appear in Ann. Inst. Poincare.

19. M.Efendiev and S.Zelik, Upper and Lower Bounds for the Kolmogorov Entropy of the Attractor for an RDE in an Unbounded Domain, JDDE 14 (2002), no. 2, 369-403.

20. E.Feireisl, Ph.Laurencot, F.Simondon, H.Toure, Compact Attractors for Reaction-Diffusion Equations in $R^{n}$, C.R. Acad. Sci Paris Ser.I319 (1994), 147 - 151.

21. E.Feireisl, Bounded Locally Compact Global Attractors For Semilinear Damped Wave Equations on $\mathbb{R}^{n}$, Differential and Integral Equations 9(5) (1996), 1147-1156.

22. Th.Gallay and S.Slijepčević, Energy Flow in Formally Gradient Partial Differential Equations in Unbounded Domains, JDDE 13 (2001), no. 4, 757-789.

23. J.Ginibre and G.Velo, The Cauchy Problem in Local Spaces for the Complex Ginzburg-Landau Equation I. Compactness Methods, Physica D 187 (1996), 45-79.

24. J. Hale, Asymptotic Behavior of Dissipative Systems, Math. Surveys and Mon., 25, Amer. Math. Soc., Providence, RI (1987).

25. A. Katok and B. Hasselblatt, Introduction to the Modern Theory of Dynamical Systems, Cambridge University Press, 1995.

26. K. Kirchgässner, Wave Solutions of Reversible Systems and Applications, J. Diff. Eqns. 45 (1982), 113-127.

27. A.Kolmogorov and V.Tikhomirov $\varepsilon$-entropy and $\varepsilon$-capacity of Sets in Functional Spaces, In: Selected works of A.N. Kolmogorov Vol III, ed., Dordrecht: Kluver (1993).

28. O.Ladyzhanskaya, V.Solonnikov and N.Uraltseva, Linear and Quasilinear Equations of Parabolic Type, M.: Nauka, 1967. 
29. S.Merino, On the Existence of the Global Attractor for Semilinear RDE on $R^{n}$, Journal Diff. Equation 132 (1996), 87-106.

30. A. Mielke, The Ginzburg-Landau Equation in its Role as a Modulation Equation, Handbook for Dynamical System (ed. by B.Fiedler), Elsevier (2002), 759-834.

31. A. Mielke, The Complex Ginzburg-Landau Equation on Large and Unbounded Domains: Sharper Bounds and Attractors, Nonlinearity 10 (1997), 199 - 222.

32. A. Mielke, Bounds for the Solutions of the Complex Ginzburg-Landau Equation in Terms of the Dispersion Parameters, Physica D 117 (1998), 106 - 116.

33. A. Mielke and G.Schnider, Attractors for Modulation Equations on Unbounded Domains Existence and Comparison, Nonlinearity 8 (1995), 743 - 768.

34. R. Temam, Infinite-dimensional dynamical systems in mechanics and physics, Springer-Verlag, New-York, 1988.

35. H. Triebel, Interpolation Theory, Function Space, Differential Operators, North-Holland, Amsterdam-New York, 1978.

36. M.Vishik and S.Zelik, The Regular Attractor for a Nonlinear Elliptic System in an Unbounded Domain, Mat. Sbornik 190 (1999), no. 6, 23-58.

37. E. Zeidler, Nonlinear Functional Analysis and its Applications. Part I. Fixed-Point Theorems, SpringerVerlag, 1985.

38. S.Zelik, The Attractor for an Nonlinear Reaction-Diffusion System in $\mathbb{R}^{n}$ and the Estimation of it's ع-entropy, Math. Notes 65 (6) (1999), 941-943.

39. S.Zelik, The Attractor for a Nonlinear Reaction-Diffusion System in an Unbounded Domain and Kolmogorov's Epsilon-Entropy, Math. Nachr. 232 (2001), no. 1, 129-179.

40. S.Zelik, The Attractor for a Nonlinear Reaction-Diffusion System with a Supercritical Nonlinearity and it's Dimension, Rend. Accad. Naz. Sci. XL Mem. Mat. Appl. 24 (2000), 1-25.

41. S.Zelik, The Attractor for a Semilinear Damped Hyperbolic Equation in $\mathbb{R}^{n}$ : Dimension and E-entropy, Math. Notes 67(2) (2000), 304-307.

42. S.Zelik, The Attractor for a Nonlinear Hyperbolic Equation in an Unbounded Domain, Disc. Cont. Dyn Sys. Ser. A 7 (2001), no. 3, 593-641.

43. S.Zelik, The Attractors of Reaction Diffusion Systems in Unbounded Domains And Their Spatial Complexity, DANSE FU-Berlin, preprint 32/00 (2000).

44. S.Zelik and A.Mielke, Attractors of Reaction-Diffusion Systems in $\mathbb{R}^{n}$ with Strictly Positive Spatio-Temporal Topological Entropy, in preparation (2002). 